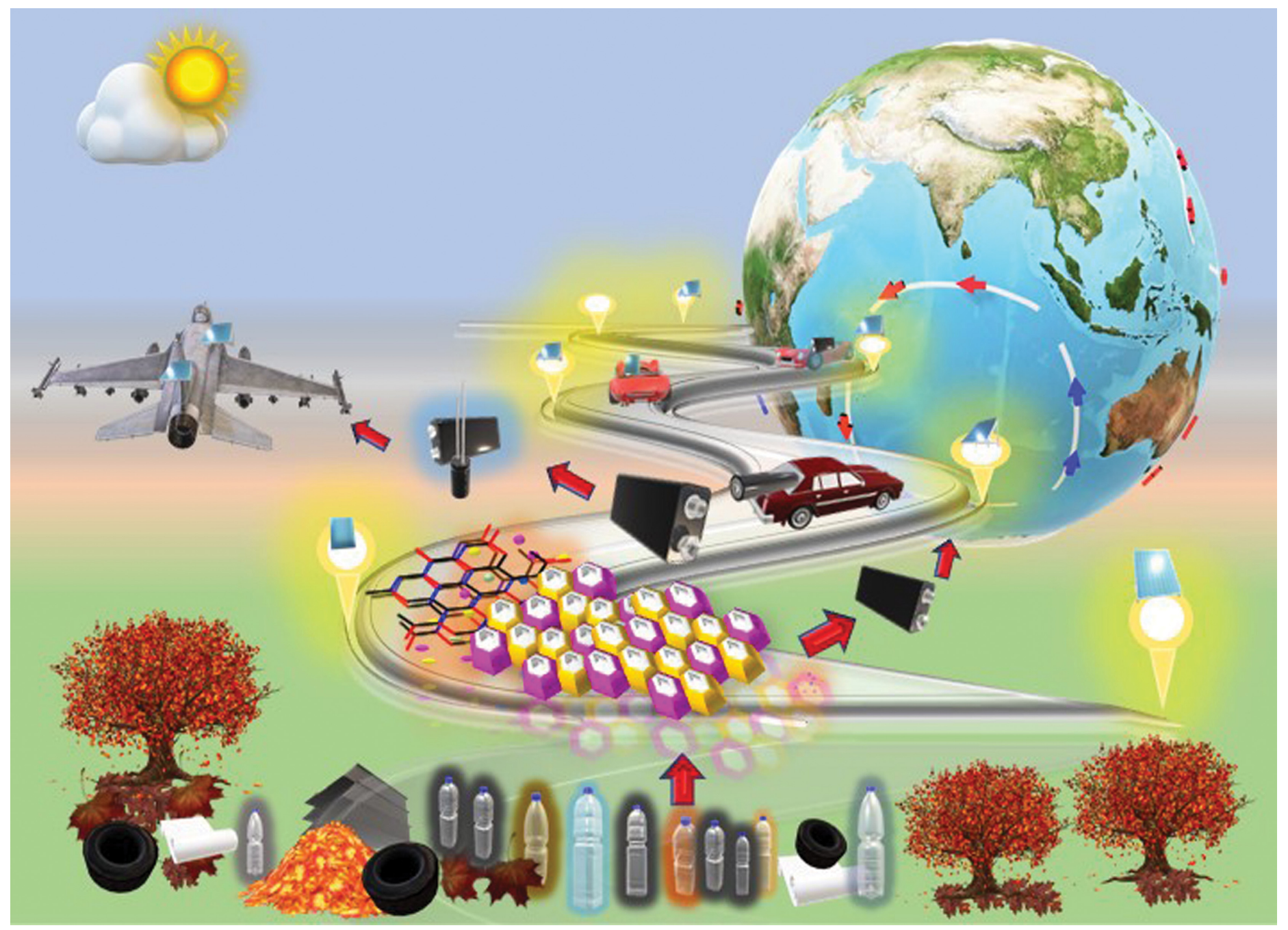

Showcasing a promising research prospects of solid waste derived carbon nanomaterials for energy applications by Professor N. G Sahoo's research group at PRS-NSNT Centre, Department of Chemistry, D.S.B. Campus, Kumaun University, Nainital, Uttarakhand, India.

Solid waste-derived carbon nanomaterials for supercapacitor applications: a recent overview

Fruitful management of solid waste materials and increasing demands of energy storage devices are the most challenging issues in the world. However, the upcyclization of solid waste into carbon nanomaterials for supercapacitors applications demonstrates an effective method to deal with these issues. The carbon nanomaterials are well recognised as the potential multidimensional materials to be used in energy applications. These supercapacitors with superior properties such as highpower density, outstanding charge-discharge rates, and energy density can definitely accomplish the demand related to energy requirements for futuristic generations.

\section{As featured in:}

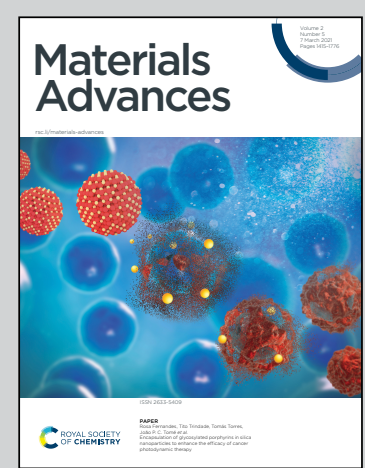

See Nanda Gopal Sahoo et al., Mater. Adv., 2021, 2, 1454 
Check for updates

Cite this: Mater. Adv., 2021,

2. 1454
Received 8th November 2020, Accepted 15th January 2021

DOI: $10.1039 / \mathrm{d} 0 \mathrm{ma} 00871 \mathrm{k}$

rsc.li/materials-advances

\title{
Solid waste-derived carbon nanomaterials for supercapacitor applications: a recent overview
}

\author{
Gaurav Tatrari, Manoj Karakoti, Chetna Tewari, Sandeep Pandey, \\ Bhashkar Singh Bohra, Anirban Dandapat (D) and Nanda Gopal Sahoo (D) *
}

\begin{abstract}
Universal solid waste management and its hazardous effects on the ecology, ecosystem, and the global human health index are some of the major issues that are currently threatening our future. The overproduction of solid waste materials is mainly the consequence of human population explosion and extraordinary increase in global economy along with global productivity. Due to the rapid growth in global population and the related demands, the production of solid waste materials is increasing day by day. In order to have a better solution to solid waste management, the best ethical way is to convert solid waste materials into valuable carbon-based nanomaterials (CNMs) such as CNT, graphene, and carbon quantum dots (CQDs), which can further work as precursors in energy storage devices. This critical review summarizes the recent progress in the structural forms and current situational analysis of universal solid waste based CNMs. It also includes broadly discussed issues, starting with the current waste situation analysis of global energy crisis and the production of graphene, including its counterparts, as the precursors for supercapacitor applications. A paradigm study of various solid waste derived CNMs with their significance, which, in particular, depends upon the types of CNMs and the classification of solid waste derived CNMs on the basis of different types of pore size and pore distribution, has been carried out. The various structural modifications and their effects on the electrochemical properties have been discussed thoroughly for supercapacitor applications including their future possibilities and scope. The review outlines the crucial mechanism followed during the charge storage of different kinds of supercapacitors and various electrochemical calculations involved during the performance testing of the fabricated device. We hope that the target audience, belonging to the energy or material science field, may acquire relevant information from this review, which may further help them to design futuristic studies in the field.
\end{abstract}

\section{Introduction}

Global population explosion has led an the exponential and unorthodox growth in universal development and thus, the related demands are also increasing day by day, which lead to an exponential increase in the production of different kinds of solid wastes. According to some recent scientific articles, globally, a total of 7 to 8 billion tons solid waste is being produced every year, which has several harmful environmental and health effects, which create a huge challenge for the scientific community. ${ }^{1}$ Waste plastic typically weighs $10 \mathrm{wt} \%$ of the global waste generated per year. ${ }^{2}$ In the last 50 years, universal contamination due to plastic waste has increased dramatically to its extreme level, mainly due to its applications in various industries, such as packaging, building and construction,

Prof. Rajendra Singh Nanoscience and Nanotechnology Centre, Department of Chemistry, DSB Campus, Kumaun University, Nainital, Uttarakhand, India.

E-mail:ngsahoo@yahoo.co.in transportation, automobile energy storage devices, and electronic goods. In 2011, universal plastic production reached at the highest record value of 280 million tons, to which $23 \%$ was contributed by China alone, followed by $21 \%$ from the European Union, 16\% from USA, and 5\% from Japan. ${ }^{3}$ Solid biomass and agricultural wastes generate many toxins on burning that are usually released into the atmosphere. The nature of emission mainly depends upon the kind of feedstock of biomass and their types. Several pollutants such as nitrogen oxide, carbon monoxide, and sulfur dioxide are commonly released in the atmosphere. Waste biomass causes forest fires and can also increase the global production of greenhouse gases by 25 to $30 \%$ each year. ${ }^{4-10}$ According to some reports, universal crops yield about 146 billion tons of solid waste every year $^{11}$ and the household waste is about 100 billion tons. ${ }^{12}$ The proper management of such a huge amount of solid waste is itself a big environmental issue. ${ }^{13}$ To overcome the situation with great success, scientific development needs an innovative approach, which can solve and manage the utilization of such 
wastes by adopting some advanced scientific technologies. In this regard, the existence of nano-science and nanotechnology has been a huge boon for the scientific community, which can work simultaneously as the lifeline for the sustainable management of several universal obligations, including solid waste management and the universal energy crisis. Accordingly, waste management technologies need to be innovated so that the problem of solid waste managements can be dealt with comprehensively. In addition, the major problem in front of the scientific community is to develop a new pathway for the production of eco-friendly and cost-effective energy storage devices to deal with the energy crisis of the world. Meanwhile, dealing with these global issues, several scientific communities have been able to report some distinct researches, including efficient waste management and cost-effective production of carbon nanomaterials (CNMs), for e.g., carbon nanotubes (CNT), graphene, and carbon quantum dots (CQDs), for various industrial applications, especially in the field of energy. CNMs are carbon-based nanomaterials having extensive potential for various applications. Thus, the generated solid waste can be utilized by increasing the production of various CNMs from solid waste materials. Among CNMs, CNTs, CQDs, and graphene have some exceptional properties, which includes a huge aspect ratio, supreme electrical properties, exceptional mechanical strength, and superior thermal conductivity. ${ }^{14-17}$ The research articles published during 2014-2019 on the conversion of solid wastes into CNMs and related applications such as supercapacitors, solar cells, fuel cells, drug delivery, and bioimaging are outlined in Fig. 1. It clearly shows the extensive research that is taking place in the field of waste management and solid waste based CNMs. It also represents the growing futuristic interest among the scientific community for solid waste based CNMs. The evaluated data was searched in Feb. 2020 with the help of Web of Science. The successive increment in per year research publications on solid waste based CNMs, especially for their use in the field of energy, i.e., supercapacitor applications, show the increasing number of publications per year (Fig. 1).

Fig. 2 clearly shows the current trends and research direction involving different kinds of solid waste based CNMs, which indicates the role of waste management in futuristic development and innovations. The presence of high carbon content in

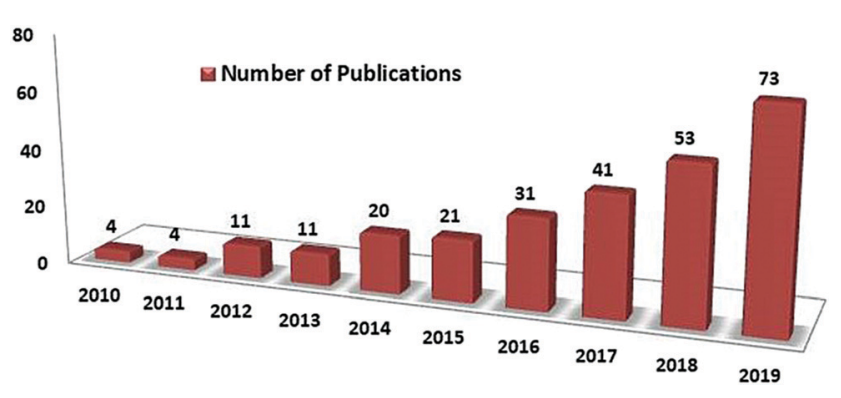

Fig. 1 Number of publications per year reported on waste based CNMS from 2010 to 2019 (using Web of Science database; date of search Feb. 2020).

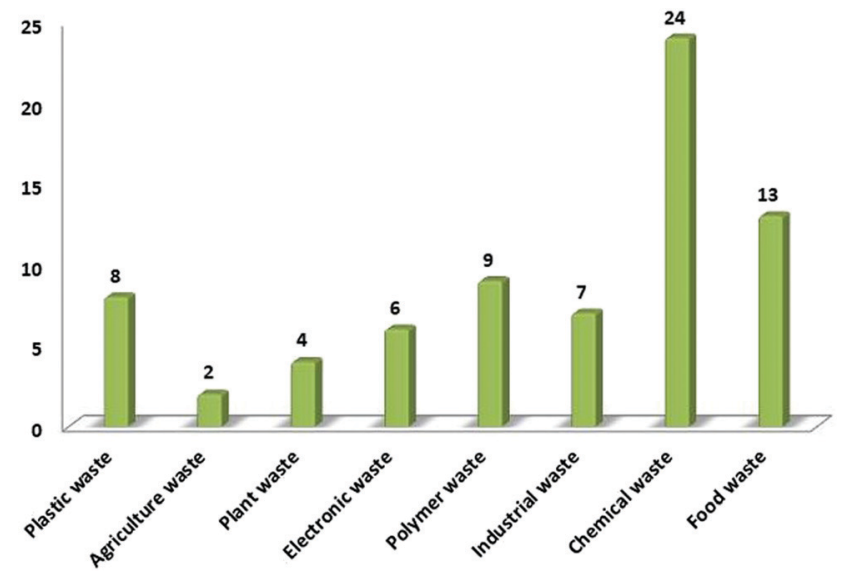

Fig. 2 Graphical representation of the published research articles on different kinds of solid waste based CNMs during the years 2014-2019 (using Web of Science database; date of search February 2020).

solid waste materials allows the scientific community to convert them into different CNMs such as graphene, CNT, and CQDs; further, modified and doped nanomaterials have been used as materials in energy appliances extensively by various researchers in the last few years.

The applications of CNMs in various fields of science and technology are due to their respective excellent properties and surface modifications along with the specific properties, which have also been assisted thoroughly in various applied fields (Fig. 3), such as supercapacitor materials, ${ }^{18-20}$ solar cells, ${ }^{21-23}$ as a battery's electrode, ${ }^{24,25}$ catalyst supports systems for proton exchange membrane fuel cells, ${ }^{26-28}$ and also to develop polymer nanocomposites for various applications, ${ }^{29-31}$ i.e., in drug delivery, bioimaging, ${ }^{32-34}$ medicinal biology, ${ }^{35-38}$ aerospace, ${ }^{39,40}$ and new age weapon technology. ${ }^{41,42}$

Numerous theoretical approaches have been made by researchers for the analysis of waste based CNMs utilized in various applications by functionalization and doping possibilities

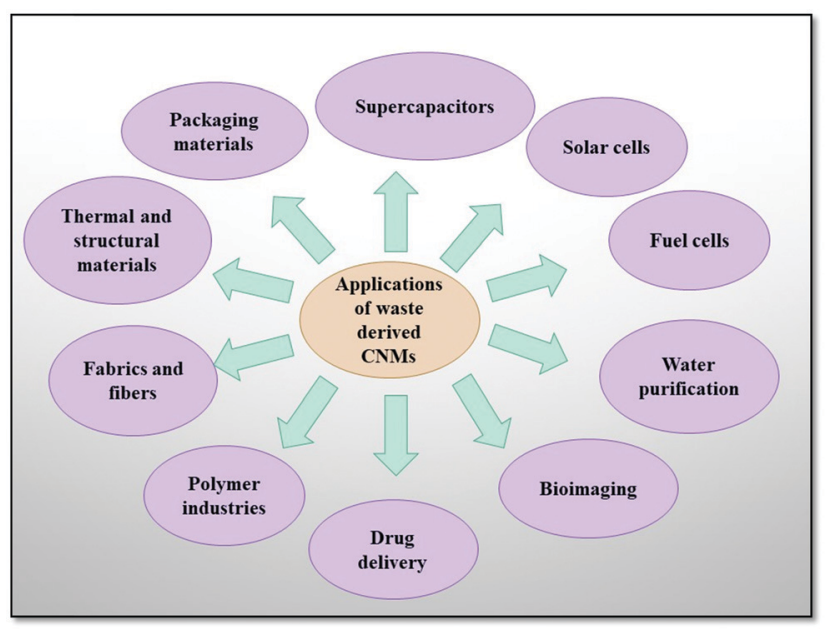

Fig. 3 Schematic representation of the applications of waste materialderived CNMs. 
with various metals, ${ }^{43,44}$ metal oxide, ${ }^{45,46}$ non-metals, ${ }^{47,48}$ or by strong surface interactions with organic reagents, ${ }^{49,50}$ conductive polymer incorporation, ${ }^{51,52}$ etc. Moreover, it has been reported that functionalized CNMs are stronger candidates for various uses, in comparison to non-functionalized ones, as they have aggregation problems. ${ }^{53,54}$ Based on the structure and elemental doping, CNMs have been vigorously used in electrode materials of batteries, supercapacitors, and fuel cells for various biomedical applications.

Supercapacitors have been sub-divided as the electrochemical double layer (EDLC) and pseudo supercapacitors. ${ }^{55,56}$ The use of CNMs as electrode materials for EDLC and pseudo supercapacitors have been rising since the last decade due to the exuberant power density and excessive energy density they produce, especially for various energy applications in comparison to conventional batteries and Li-ion batteries. ${ }^{57-59}$ In EDLC, the electrical energy is stored through the electrostatic accumulation of charge and in pseudocapacitors through reversible reactions. The electrical conductivity, theoretical specific surface area, geometrical configurations, porous nature, and variations in mass densities excessively affect the performance of supercapacitors.

Nowadays, structural modifications are also building up some hype in the field; as recently found, flexible supercapacitors are emerging as a winner in comparison to conventional fixed-batch devices as they have been successfully implemented in various devices such as in portable electronic devices, electrical vehicles, and memory backup systems. ${ }^{60-62}$ Thus, by such a theoretical evaluation of universal waste management situations and universal energy requirements, it can be outlined that the solid waste based CNMs are the best way to design proficient technology having economic and ecofriendly benefits.

This review highlights the remarkable properties of various solid waste based CNMs and their successive utilization in energy storage applications. The recent development and innovations to deal with universal solid waste management and innovative structural modifications of CNMs to the enhance electrochemical performance of the fabricated device based on their unique properties are also included. In conjunction, the specific study and performance about cost-effective supercapacitors with specific reference to their structural modifications, degree of carbonizations, pore structure, surface properties, doping, and variations in mass densities have been discussed. The review also includes various scientific methodologies followed during performance testing and the instrumentation required. In addition, various new developments have been discussed in universal waste management and universal energy crisis to make this review a valuable tool for the future perspective of mankind.

\section{Solid waste as a universal problem}

Solid waste has emerged as a universal problem mainly due to its harmful environmental aspects and health hazardous consequences. Continued population growth and their increasing universal demands are the main reason behind the overproduction of various solid wastes such as waste plastics, agriculture waste, chemical waste, paper waste, industrial waste, and electronic waste. Solid waste has emerged as an extremely dangerous factor that affects the human health and the environment simultaneously. It causes diseases such as cancer, skin disease, respiratory disease, birth defects, and reproductive disorders. ${ }^{63,64}$ According to Biggeri et al., ${ }^{65}$ the risk of having lung cancer increases on decreasing the distance of the source from the incinerator. Comba et al. ${ }^{66}$ reported a case-controlled study on the effect of solid waste materials on human health. They reported a significant risk of cancer and other diseases in residents living within $2 \mathrm{~km}$ of the solid waste dumping sites. ${ }^{66}$ On the other hand, Zambon et al. ${ }^{67}$ reported a clear association in between soft tissue sarcoma and dioxin exposure at the municipal solid waste incinerators site. Several other diseases have also been reported as the side effects of solid waste sites, ${ }^{68}$ which includes cancer, stomach ache, liver damage, and lung cancer. Hsiue et al. reported a study on the respiratory health issues of children living in polluted areas, where a total of 384 children were chosen from polluted areas. The successive decrement in relative pulmonary function was reported for most of the children. ${ }^{69}$ Similarly, a comprehensive study was done by Miyake et al. ${ }^{70}$ on school children. They observed a relationship amongst the frequency of allergic sicknesses and symptoms in children with the distance from the polluted areas, where the children studying at schools nearer to incineration plants were reported to have more frequent headaches and wheezing. Along with this, poor waste management is also a crucial factor in polluting the oceans, blocking drains, triggering flooding, and harming animals that eat waste naively, thus disturbing environmental and economic development.

Table 1 depicts the contribution of various provinces worldwide in the production of waste plastic and Fig. 4 shows the distribution of solid waste in India. According to the World Bank's report waste 2.0, universally, 2.01 billion tonnes of solid waste is generated annually, in which at least 33\% is not environment friendly. According to the World Bank report, rapid urbanization, population explosion, and economic growth will push universal solid wastes so as to increase their content by $70 \%$ in the coming 30 years by generating 3.40 billion tonnes of solid waste annually. Waste plastic alone

Table 1 The contribution of various provinces worldwide in the production of waste plastic 3,71

\begin{tabular}{lll}
\hline S. no. & Province & Plastic production in \% \\
\hline 1. & China & 23 \\
2. & Europe & 21 \\
3. & Rest Asia & 20 \\
4. & Middle east Africa & 7 \\
5. & Latin America & 5 \\
6. & Japan & 5 \\
7. & CIS & 3 \\
8. & NAFA & 20 \\
9. & USA & $\mathbf{1 6}$
\end{tabular}




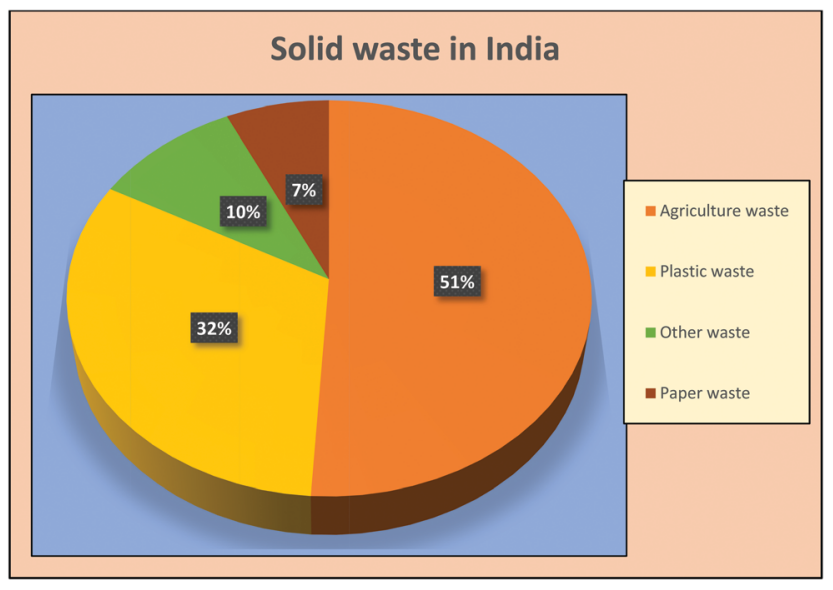

Fig. 4 The distribution of solid waste in India. ${ }^{72-74}$

makes up to $90 \%$ of marine junk and chokes most of the oceans. In the year 2016, 242 million tonnes of plastic waste was generated globally, which is equivalent to 24 trillion $500 \mathrm{~mm} 10 \mathrm{~g}$ plastic bottles. The water volume of such bottle could fill up 2400 Olympic stadiums, 4.8 million Olympic size swimming pools, or 40 billion bathtubs. This is also the weight of 3.4 million adult blue whales or 1376 Empire State Buildings. ${ }^{75}$ It can be concluded that solid waste has emerged as a huge potential problem universally and the scientific community. In order to view the situation more clearly, universal solid wastes can be classified in to two subheadings, viz., carbonic and non-carbonic solid wastes. Fig. 5 shows the classification of different solid wastes present in the global perspective. In depth, the current universal solid waste situation and its distribution scenario depicts the urgency for its sustainable management. The section given below explains the possible application potentials of solid waste materials and their derivatives for the sustainable management of universal ecology.

\section{Solid wastes as precursors of various CNMs}

Solid waste includes waste plastic, agricultural waste, municipal waste, paper waste, polymeric wastage, and industrial waste, most of which consist of carbonic skeletons as the structural framework, thus enabling nano-science to operate at the ground level. On the other hand, the proficiency to operate the activation can alter the structural morphology of the carbonic skeleton, mostly depending upon the processing methodology. Fig. 6 shows different kinds of solid waste materials that are used as the precursors of CNMs.

There are various methodologies to avail the conversion of carbonic waste into CNMs, which include chemical vapour deposition (CVD), physical vapour deposition (PVD), organic redox methodologies (e.g., modified Hummers' method), and pyrolytic heating. Every methodology (Fig. 7) has its own specificity for producing different carbon nanomaterials (CNMs), which mainly depend upon specific surface reactions and variation of reagents. However, in the pyrolysis process, the heating temperature, pressure, atmosphere, and scan rate matters the most, which alters the formations of layers and variations in CNMs. This review summarizes the solid waste to CNMs conversion strategies, their application potential in supercapacitors, and other morphological effects affecting the electrochemical performance of the fabricated supercapacitor device.

\subsection{Solid waste to graphene}

Mostly, solid waste consists of a carbonic framework of atoms having millions of layers; thus, it can be converted into graphene. Graphene is a single layer of graphite that has a 2D skeleton and is the thinnest known allotropic form of carbon, with the highest specific surface area, excellent electrical and thermal conductivity, surpassing the mechanical strength ${ }^{76-79}$ due to $\mathrm{C}-\mathrm{C}$ sigma

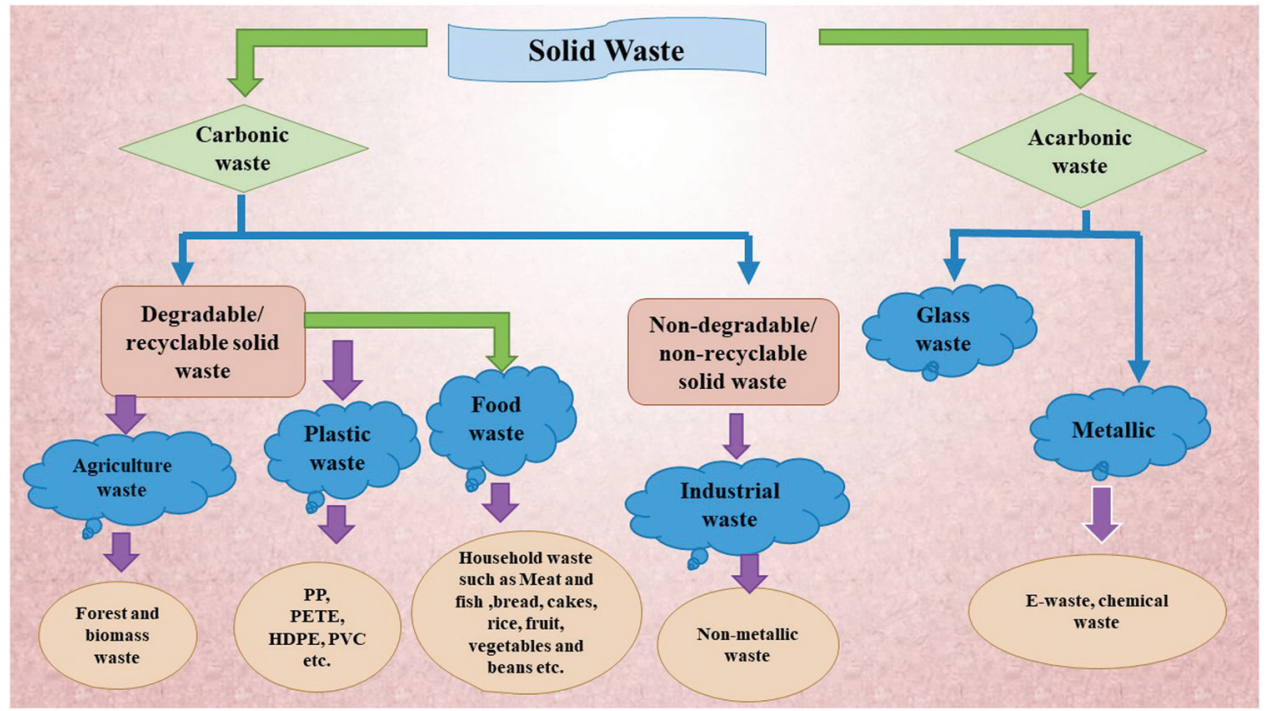

Fig. 5 Schematic illustration of the distributions of various solid wastes. 


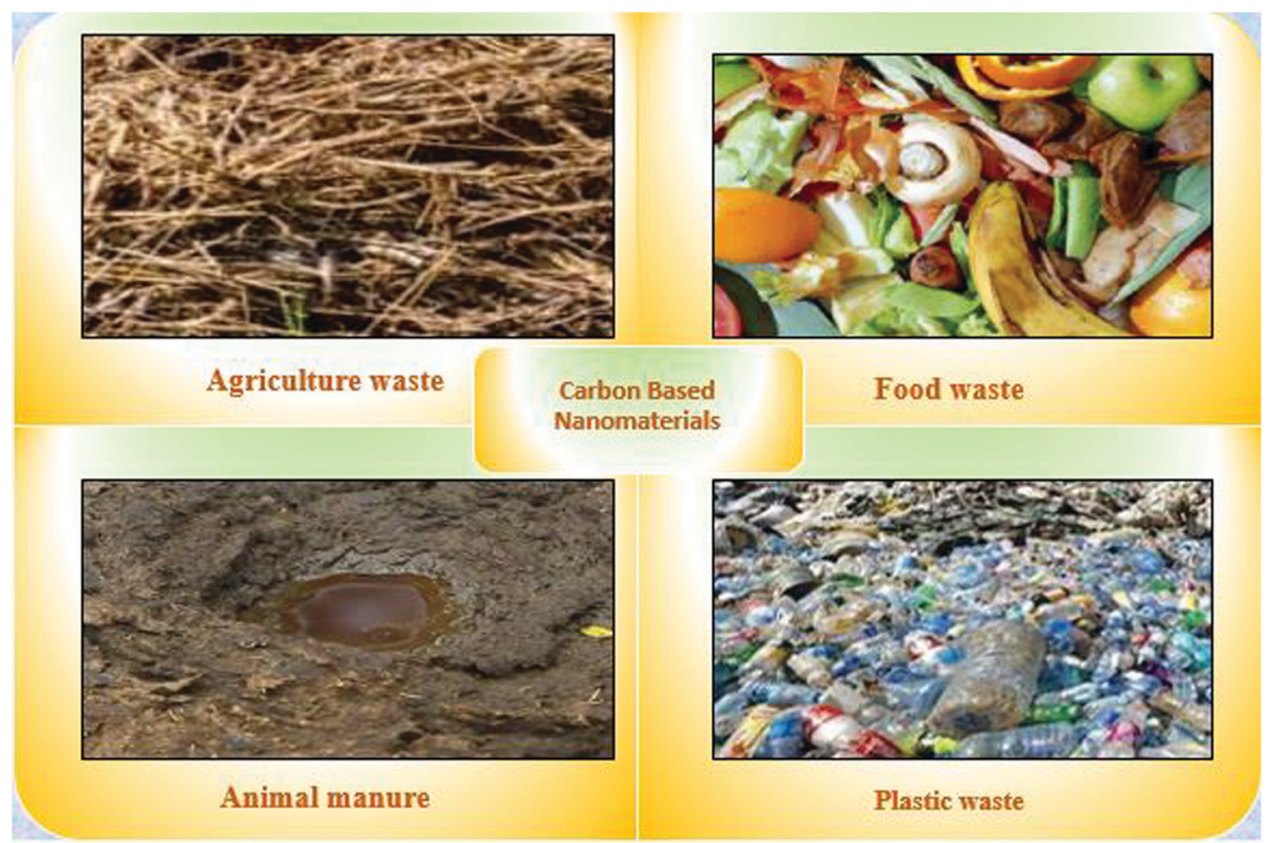

Fig. 6 Pictorial representation of the different precursors for the production of CNMs.

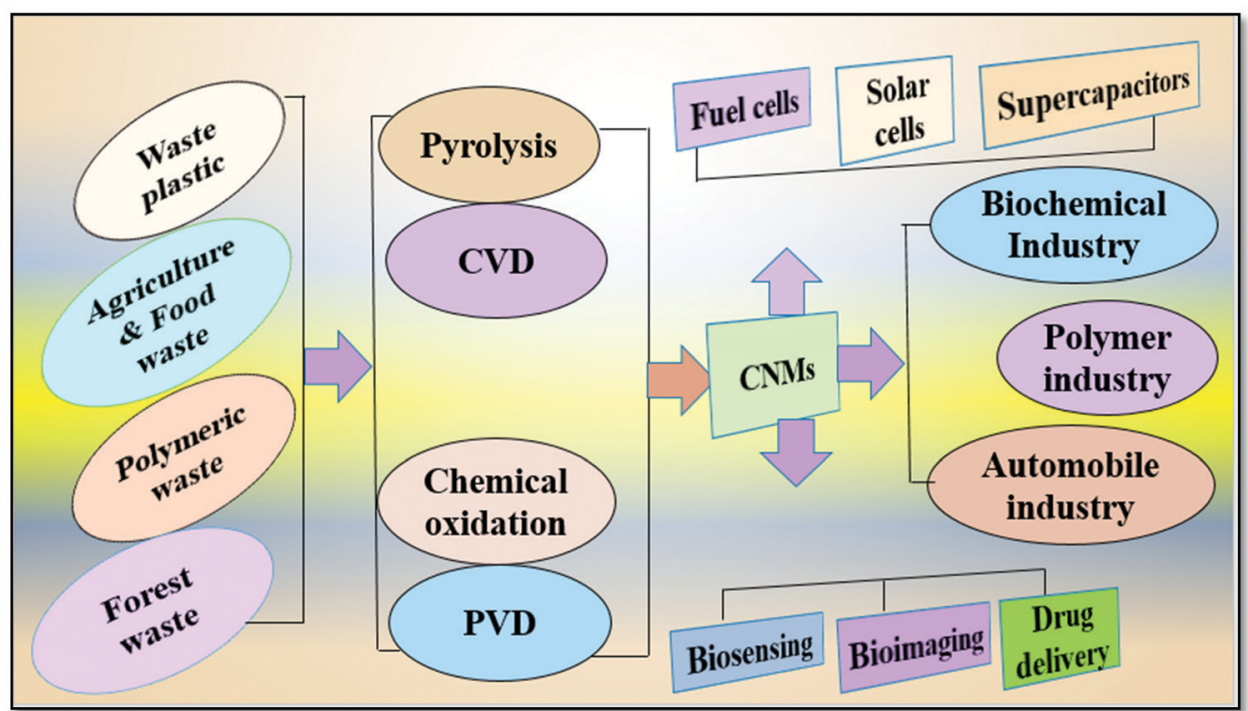

Fig. 7 Schematic representation showing various synthetic pathways and industrial uses of different waste derived CNMs.

covalent linkage, which makes graphene a brilliant candidate for its use in various applications.

Essawy et al. have synthesized graphene from waste plastic bottles containing PET plastic bottles. The plastic wastes were crushed firstly, and sieved to get micron-size particles, then the sieved residue $(2 \mathrm{~g})$ was placed inside the stainless-steel autoclave reactor (SS316), which was placed inside the center of an electric furnace at $800{ }^{\circ} \mathrm{C}$ for $1 \mathrm{~h}$ and finally cooled the sample, which was characterized as graphene. ${ }^{80}$ Gong et al. have used the up-cyclization process for the conversion of plastic waste into graphene. They have reported a simple method of synthesizing graphene flakes with high yield by introducing organically modified catalyst (OMMT), which was used as a degradation agent for waste polypropylene (PP) to graphene conversion at $700{ }^{\circ} \mathrm{C}$ inside the tube furnace under an inert environment. ${ }^{81}$

Fig. 8 displays the synthesis of porous graphitic carbon from natural cotton and the conversion of reeds into interconnected porous carbon nanosheets. In the first case, high temperature carbonization, followed by the activation through sodium metal ion and further modifying it with an organic molecule, were performed to manufacture porous graphitic carbon from natural 

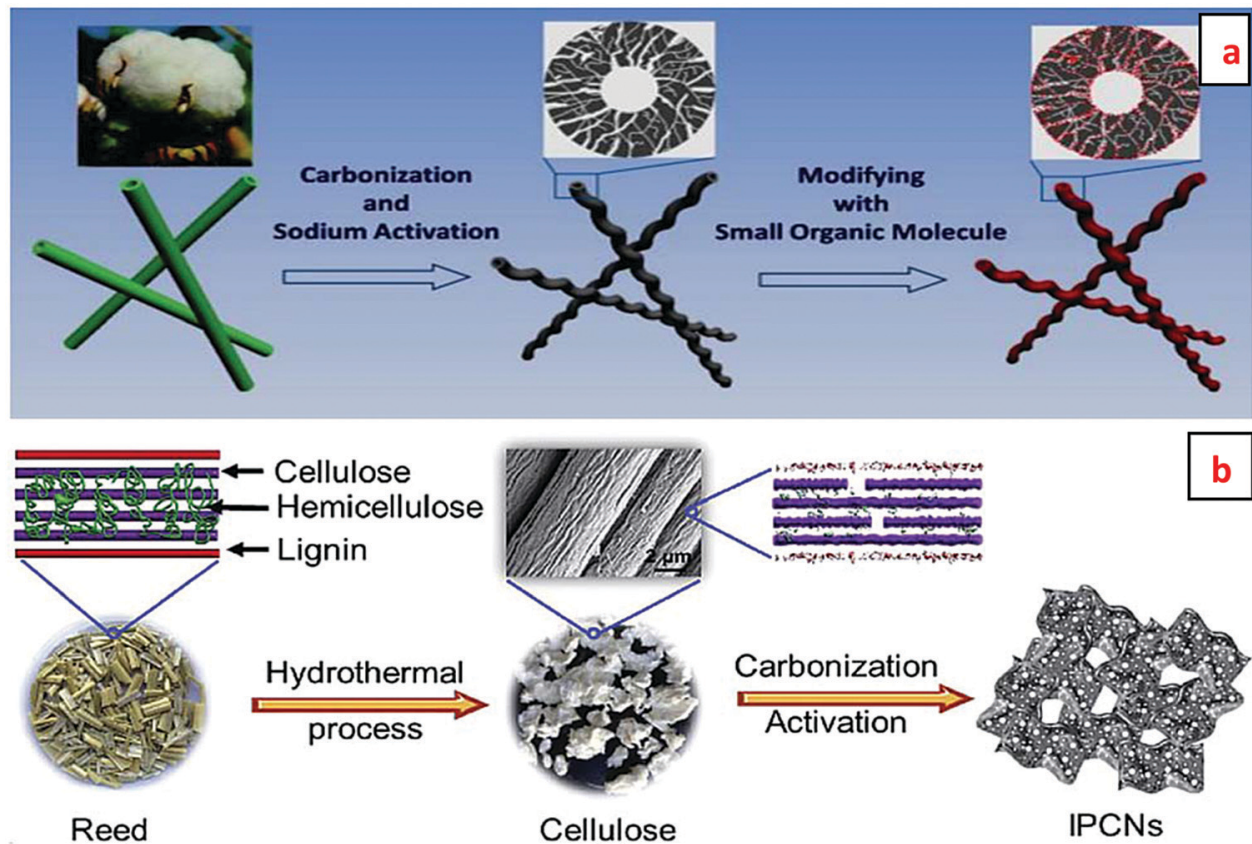

Fig. 8 Schematic illustration for (a) the synthesis of porous graphitic carbon from natural cotton. ${ }^{82}$ Reproduced by permission of Elsevier, and (b) the conversion of reeds into interconnected porous carbon nanosheets. ${ }^{83}$ Reproduced by permission of RSC.

cotton. $^{82}$ On the other hand, Fig. $8 \mathrm{~b}$ depicts the processing of cellulosic reed by the hydrothermal approach, followed by carbonization and activation to produce interconnected porous carbon nanosheets. ${ }^{83}$

Kamali et al. have reported the pyrolysis of waste plastic bottles in a tube furnace at $1300{ }^{\circ} \mathrm{C}$ with the heating rate of $10{ }^{\circ} \mathrm{C} \mathrm{min}^{-1}$, followed by for overnight air drying with heating at $80{ }^{\circ} \mathrm{C}$, which converts waste plastics into the graphene nanostructure. ${ }^{84}$ Recently, Wen et al. unveiled the conversion of waste plastic into graphene nanostructure using organicallymodified montmorillonite (OMMT) clay as the catalyst and solvolytic $\mathrm{KOH}$ as the activation agent. OMMT was added in the mass ratio of $1: 5$ in the mixture at $190{ }^{\circ} \mathrm{C}$ for $5 \mathrm{~min}$, followed by carbonization in a conventional quartz tube reactor for $10 \mathrm{~min}$ at $700{ }^{\circ} \mathrm{C}$ to obtain the resultant CNM/MMT composite. Further, washing with nitric acid and HF delivers the purified form of graphene. However, for obtaining potassium-doped CNMs, the activation process followed by heating at $850{ }^{\circ} \mathrm{C}$ temperature and $\mathrm{KOH}$ solution as the activator were used. ${ }^{85}$

Recently, Pandey et al. reported a robust and innovative approach to deal with waste plastic problem by the mass conversion of waste plastic into graphene sheets, following the sophisticated two-step pyrolysis process (Fig. 9). In the first step, slow pyrolysis was carried out in a primary reactor at $400{ }^{\circ} \mathrm{C}$ with a heating rate of $5{ }^{\circ} \mathrm{C} \mathrm{min}^{-1}$. At this temperature, lower hydrocarbons were released in the form of fuels and gases. In the second step, high temperature pyrolysis at $750{ }^{\circ} \mathrm{C}$ was conducted with the heating rate of $10{ }^{\circ} \mathrm{C} \mathrm{min}{ }^{-1}$ in an inert environment. The final product was confirmed to be graphene nano-sheets. ${ }^{86}$ In another report, Tewari et al. reported an eco-friendly and green approach for the synthesis of potassium (K)-doped graphene oxide from the agricultural waste of oak seeds via solvothermal process using ethyl alcohol and distilled water as green solvents. The internal part of oak seeds was used and heated at $120{ }^{\circ} \mathrm{C}$ for $4 \mathrm{~h}$ inside the oven until a brown residue was obtained. The brownish residue was again crushed and mixed in $30 \mathrm{~mL}$ distilled water, followed by the stirring of $30 \mathrm{~min}$, has been discussed. Finally, filtration was done by using 0.2-micron pore-sized nylon filter paper. The obtained filtered liquid was dried at $100{ }^{\circ} \mathrm{C}$ and thus the obtained material was identified as potassium-doped graphene oxide. ${ }^{87}$

The conversion of halogen based solid waste materials, especially PTFE, PVDF, and PVC into nano-porous carbon, using zinc powder as a hard template, was reported by Chen et al. ${ }^{88}$ The mass ratio between the plastics and zinc powder as well as the carbonization temperature play a crucial role in determining the carbonic structure and the resultant electrochemical performances. The optimized product was obtained by carbonizing polytetrafluoroethene and zinc powder (mass ratio of $1: 3$ ) at $700{ }^{\circ} \mathrm{C}$ under the inert atmosphere of argon. ${ }^{88}$

The conversion of solid waste into CNMs followed the CVD process, as reported by various research groups. Briefly, properly washed solid waste materials were placed into a tube furnace having high temperature and an inert atmosphere. Further, the scan rate of $150 / 25 \mathrm{sccm}$ acted as a very effective parameter for the synthesis of graphene. Thus, the treated solid waste was processed at $1050{ }^{\circ} \mathrm{C}$ for 2 hours in the tube furnace just to remove the presence of oxide functionalities; finally, the annealing process resulted in few layered graphene. The scan rates of the gases may be one of the factors behind the 


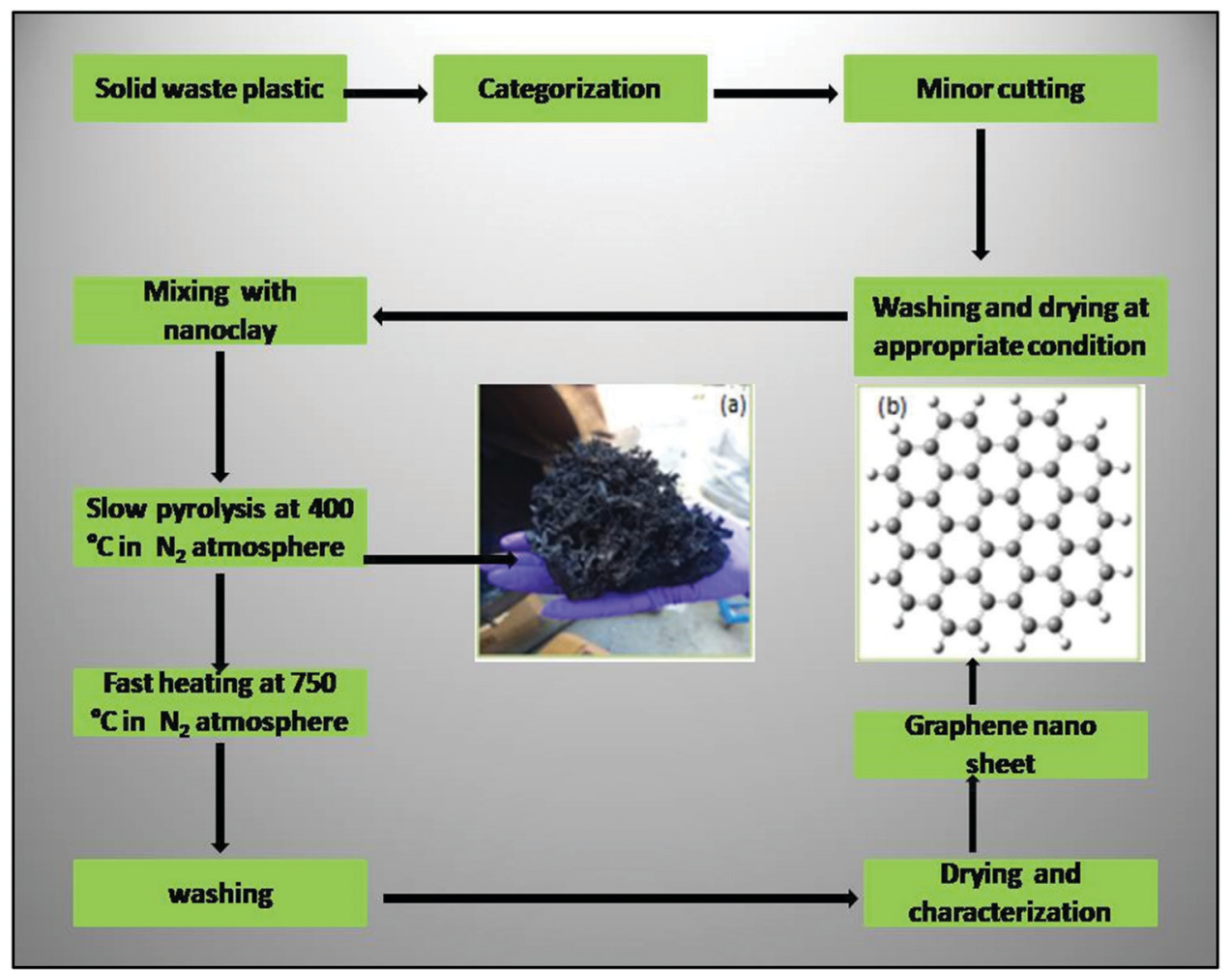

Fig. 9 Flow chart for the conversion of solid plastic into CNMs. (a) The carbon material obtained from the low temperature pyrolysis of waste plastic, (b) Skeleton structure of the graphene nanosheets' high temperature pyrolysis. ${ }^{86}$ Reproduced by permission of Elsevier.

good graphitization of solid waste materials at such a high temperature. ${ }^{89}$

Papon et al. reported the pyrolysis of carbonic waste in quartz furnace using $\mathrm{Cu}$ foils and a heating range of 1050$1070{ }^{\circ} \mathrm{C}$ with a specific gas flow of 100 square cubic centimeters of hydrogen in both the pre-annealing and annealing processes. They found that the gas flow of $98 \mathrm{sccm}$ for argon and $2.5 \mathrm{sccm}$ for hydrogen produces good quality graphene. ${ }^{90}$ Cui et al. reported the CVD method for the production of graphene from waste plastic, with argon and hydrogen as the continuously flowing gases. They used various waste plastics, such as PET, PVC, PP, PMMA, and PS and Ni foil as the catalyst. Ni foil was initially heated in an $\mathrm{Ar} / \mathrm{H}_{2}$ atmosphere at $1050{ }^{\circ} \mathrm{C}$ for half an hour to remove the oxides from the Ni surface. Thereafter, the main annealing process was carried out by following the CVD method at $1050{ }^{\circ} \mathrm{C}$ to produce free-standing graphene foil. ${ }^{91}$ Sharma et al. have used the solid waste plastic as a precursor for the production of graphene by the CVD methodology; further, Raman and other characterization techniques were used to identify the prepared product as graphene. ${ }^{92}$

\subsection{Solid waste to CNT}

Solid waste can also be converted into carbon nanotubes (CNT) by variations in the temperature, pressure, and scan rates of the pyrolysis unit. On the basis of their structural alignment, CNT is of two types: single walled carbon nanotubes (SWCNTs) and multi-walled carbon nanotubes (MWCNTs). The former one has a cylindrical roll of graphene sheets with in-phase and out-phase bonding of $\mathrm{sp}^{2}$ carbon atoms. ${ }^{93,94}$ SWCNTs are categorized in accordance to their structural patterns, conductivity, and chirality basis into metallic SWCNTs, semiconductor SWCNTs, and semi-metallic SWCNTs. ${ }^{94,95}$ The co-dimensional arrangement of SWCNTs leads to MWCNTs, whereas the difference of diameter is also huge for MWCNTs in compared to SWCNTs. ${ }^{96}$ The stronger carbon-carbon sigma bonds lead to molecular stiffness and toughness of the CNTs. ${ }^{97}$

Ganesh et al. reported waste plastic to CNTs conversion by using $\mathrm{Ni} / \mathrm{Mo} / \mathrm{MgO}$ as the catalyst. The specific activity of three components $\mathrm{Ni}, \mathrm{Mo}$, and $\mathrm{MgO}$ was measured and found to be interdependent. Nonetheless, the effect of $\mathrm{Ni}$ was found to be the most efficient one. The catalyst having a composition of $\mathrm{Ni}_{0.8} \mathrm{Mo}_{0.1} \mathrm{MgO}_{0.1}$ produces optimum yields of CNT. ${ }^{98}$

Mishra et al. used thermal heating for the conversion of municipality solid waste into CNTs using Ni as the catalyst. They used $10 \mathrm{~g}$ of $\mathrm{PP}$ as the precursor and $1 \mathrm{mg}$ of $\mathrm{Ni}$ as the catalyst and further introduced them into a tube furnace under $10 \mathrm{sccm}$ of hydrogen along with $90 \mathrm{sccm}$ for argon. The temperature of the furnace ranged from 600 to $800{ }^{\circ} \mathrm{C}$ and the product obtained at $800{ }^{\circ} \mathrm{C}$ was found to be the purest. ${ }^{99}$ Similarly, the pyrolytic approach for the production of CNTs from solid waste plastic has been explained by Pol et al. where they used $1 \mathrm{~g}$ polyethene waste with cobalt acetate as the catalyst placed into an autoclave in nitrogen atmosphere. The furnace was raised to the temperature of $700{ }^{\circ} \mathrm{C}$ and they reported $0.4 \mathrm{~g}$ CNTs as the final product along with emissions 
of some LPG gases, which were said to be responsible for the generation of pressure inside the vessel. ${ }^{100}$

As reported by $\mathrm{Wu}$ et al., the gasification of waste PP leads the formation of hydrogen gas and CNTs as major products using either $\mathrm{Ni} / \mathrm{Ca}-\mathrm{Al}$ and or $\mathrm{Ni} / \mathrm{Zn}-\mathrm{Al}$ as the catalyst. However, an enhanced amount of hydrogen was yielded by $\mathrm{Ni} / \mathrm{Zn}-\mathrm{Al}$ compared to that of $\mathrm{Ni} / \mathrm{Ca}-\mathrm{Al}$, while $\mathrm{Ni} / \mathrm{Ca}-\mathrm{Al}$ provided higher amounts of CNTs than that in the case of Ni/Zn-Al from a fixed amount of polypropylene. ${ }^{101}$ Nickel dioxides, trioxide, and hydroxides along with organically modified clay (OMMT) were used by Jiang et al. for the qualitative formation of MWCNTs at a temperature of $630-830{ }^{\circ} \mathrm{C} .{ }^{102}$ They reported a variation in the quality of CNTs due to slight variations in the temperature range and OMMT clays concentration along with Ni-based catalysts. ${ }^{102}$ One-pot direct synthesis was used by Gong et al., where heteroatom-based carbonization played a very crucial role, specially using nickel trioxide and copper chloride as the catalysts. The direct effect on the morphology and quantity of CNTs has been observed with the implementation of molar variations of each catalyst and raw materials. ${ }^{103}$

CNTs having a diameter in the range of 10-40 $\mathrm{nm}$ were obtained by the upcycling of waste carbonaceous material at the temperature of $450{ }^{\circ} \mathrm{C}$ and inert helium atmosphere. ${ }^{104}$ Curved CNTs were synthesized using Ni-plates as outstanding decomposition catalysts by Mukhopadhyay et al. ${ }^{104}$ Zhuo et al. reported the conversion of waste plastic bottle into CNTs via the up-cyclisation process, where PE and PET bottles were used under high temperature pyrolytic up-cyclization procedure, which included three steps. ${ }^{105}$ In the first step, chipped plastic bottles were placed at the temperature of $600-1000{ }^{\circ} \mathrm{C}$ under nitrogen atmosphere and lateral stage feedstock pyrolyzates were introduced into the secondary reactor with venture setup, which is the synthetic reactor, while $\mathrm{O}_{2}$ was introduced to balance the presence of the lower ratio between carbon and oxygen in the feedstock. Finally, in the third step, the introduction of catalytic substrate positioned in the direction of gaseous flow has been operated. ${ }^{105}$

Similarly, the conversion of waste PP into CNT was done by Liu et al. through binary synthetic pyrolytic approach in a screw kiln reactor. They reported pyrolysis at $1023{ }^{\circ} \mathrm{C}$ in the presence of zeolite-based catalyst for the decomposition of plastic into a moving bed reactor with an inert atmosphere. The synthesis of MWCNTs at $723-1123{ }^{\circ} \mathrm{C}$ temperatures in the presence of nickel oxide as the catalyst has also been reported, having a maximum yield of MWCNTs at $973{ }^{\circ} \mathrm{C} .{ }^{106}$ These studies showed that the nature and proportions of the catalyst play a key role in the development of CNTs from waste plastic. Some research studies have also established $\mathrm{Ni}$ as an excellent catalyst and excellent promoter for CNT production. On the other hand, the optimum quality of MWCNTs was obtained by varying the concentrations organic and inorganic catalysts and the corresponding temperature. Researchers have revealed that the optimum molar ratios of carbon and nickel can also enhance the production of CNTs, where click chemistry plays a very crucial role. A molar ratio of 0.125 for the $\mathrm{Cl} / \mathrm{Ni}$ complex results in excellent yield for CNT production. ${ }^{104}$
The implementation of different bed reactors and high temperatures for the synthesis of CNTs have been reported by various researchers, where the venture setup of the reactor was stated as the specific reactor setup, which can control the production of CNTs with specific flow rates of $\mathrm{O}_{2}$ for oxidation. The temperature of $973 \mathrm{~K}$ was specified as the maximum production temperature for multiwalled CNTs, mostly due to the distinct folding of the layers at such a high temperature. The slow or sudden rise of temperature with catalytic eminence and types of reactors could be the possible reason behind the changes in production and qualities of CNTs.

\subsection{Waste to carbon quantum dots}

CQDs have some interesting properties, such as exciting photoluminescence behavior, optically stable nature, biocompatibility, and low toxicity, which have attracted researchers worldwide. ${ }^{107-110}$ Various methodologies, i.e., arc-discharge, laser ablation, electrochemical oxidation and microwave heating, and hydrothermal methodology, have been reported for the preparation of CQDs from waste materials. ${ }^{111,112}$

In general, there are two methodologies for the synthesis of quantum dots, which includes top-down and bottom-up approaches, as depicted in Fig. 10. In the bottom-up approach, smaller molecules aggregate to form larger ones, while in the top-down approach, large molecules are broken down into their smaller counterparts for the synthesis of quantum dots. ${ }^{113,114}$ The synthetic methods follow either of these two broad categories. In top-bottom and bottom-top methods, however, the synthesis of quantum dots (QDs) from solid waste materials is usually processed through the top-bottom approach.

Wei et al. reported the synthesis of CQDs from waste paper by a simple green hydrothermal methodology, where they chopped waste paper and sonicated it with distilled water for $30 \mathrm{~min}$, then transferred it into a Teflon-lined stainless-steel reactor of $40 \mathrm{~mL}$ at $180{ }^{\circ} \mathrm{C}$ for $10 \mathrm{~h}$. A dark brown precipitate was collected by centrifugation and the obtained residue was finally dialyzed for $48 \mathrm{~h}$ with distilled water for further purification. ${ }^{115}$

$\mathrm{Hu}$ et al. reported the synthesis of CQDs from waste plastic bags by hydrothermal treatment in $\mathrm{H}_{2} \mathrm{O}_{2}$ solution, where small pieces of plastic waste were poured into a $50 \mathrm{~mL}$ stainless steel autoclave having $30 \mathrm{~mL} \mathrm{H}_{2} \mathrm{O}_{2}$ solution at $180{ }^{\circ} \mathrm{C}$ for $12 \mathrm{~h} .{ }^{116}$ Further, centrifugation was performed at $12000 \mathrm{rpm}$ for $10 \mathrm{~min}$ to remove the presence of various impurities, followed by dialysis for 3 days under a solution of deionized water to obtain the pure CQDs. $6 \mathrm{mg}$ of CQDs was dissolved in $100 \mathrm{~mL}$ PBS buffer solution and then ferrate ions $(0.1 \mathrm{~mL})$ were added into the CQDs $(0.9 \mathrm{~mL})$ dispersion to record the fluorescent emission spectra with the excitation wavelength of $320 \mathrm{~nm} .{ }^{116}$

The conversion of polyolefins into fluorescent CQDs by the green hydrothermal approach was also carried out by Kumari et al. A combination of green hydrothermal approach and chemical oxidation process produced CQDs with a very high quantum yield of $4.84 \% .^{117}$

Park et al. reported a simple sustainable large-scale approach to synthesize CQDs, where they converted $100 \mathrm{~kg}$ of 
Top-Down Method

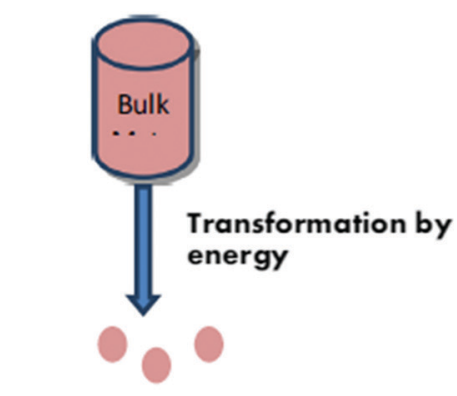

food waste into water soluble graphene quantum dots. The food waste $(100 \mathrm{~kg})$ in $500 \mathrm{~L} 10 \%$ ethanol was treated at $40 \mathrm{kHz}$ ultrasound for $45 \mathrm{~min}$, followed by centrifugation at speed of $4500 \mathrm{rpm}$ for $5 \mathrm{~min}$ to obtain uniformity in the particle size and shape, while a $0.22 \mu \mathrm{m}$ filtration membrane was utilized in the final purification of the $\mathrm{G}$ dots. The band at $400-470 \mathrm{~nm}$ confirms the synthesis of photo-luminescent $\mathrm{G}$ dots (Fig. 11). ${ }^{118}$

According to Thambiraj et al., the green synthesis of CQDs can be achieved from sugarcane bagasse by chemical oxidation, followed by the high temperature exfoliation of sugarcane biomass, where they dried sugarcane biomass in sunlight for a week, then processed it for combustion at $60{ }^{\circ} \mathrm{C}$ in an open atmosphere. After that, the residue was mixed into $200 \mathrm{~mL}$ toluene and stirred thoroughly for $24 \mathrm{~h}$ at room temperature; further sonication was done for $1 \mathrm{~h}$ along with centrifugation at $10000 \mathrm{rpm}$ for $30 \mathrm{~min}$ at room temperature, while ethanol was used for the dilution of the obtained liquid. ${ }^{119}$

CQDs were also prepared from orange waste peels via the hydrothermal carbonization method at mild temperature $\left(180{ }^{\circ} \mathrm{C}\right)$. Firstly, the orange peels were washed and oven dried at $150{ }^{\circ} \mathrm{C}$ for $10 \mathrm{~h}$, followed by washing with sulphuric acid $(0.1 \mathrm{M})$, and then oxidized by sodium hypochlorite solution at room temperature for 4 hours. ${ }^{120}$ Finally, the oxidized peels were placed under hydrothermal conditions in a Teflon-lined autoclave at $180{ }^{\circ} \mathrm{C}$ for $12 \mathrm{~h}$ to obtain the desired CQDs. In a similar way,

\section{Bottom-Up Method}
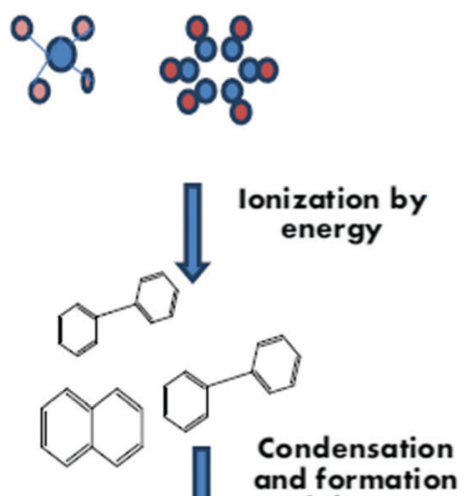
of clusters

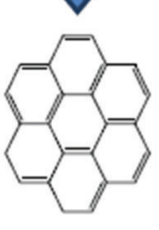

Cluster

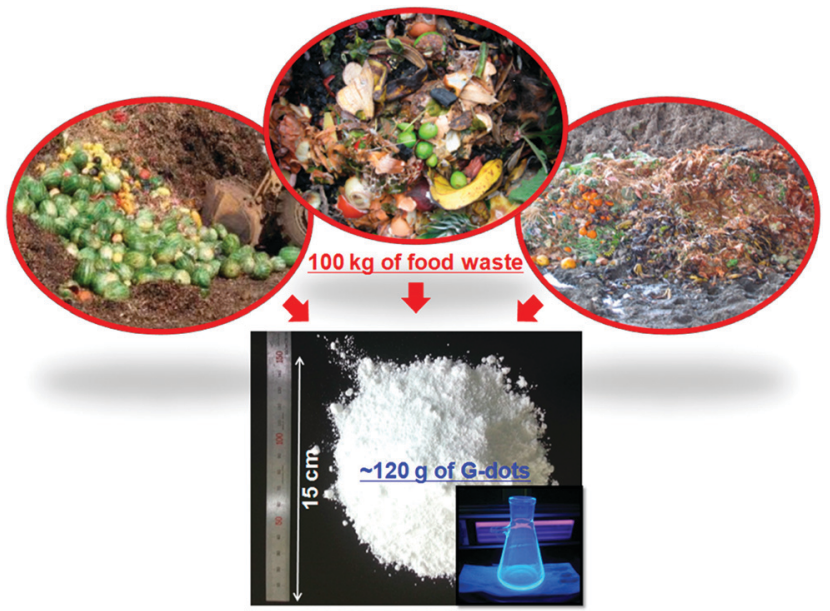

Fig. 11 Schematic description of the large-scale synthesis of G-dots. These nano-dots represent efficient transition from large food waste to valuable carbon-based nanomaterials. ${ }^{118}$ Reproduced by permission of American Chemical Society.

Tyagi et al. also obtained CQDs from lemon peel following the hydrothermal approach at $200{ }^{\circ} \mathrm{C}$ temperature ${ }^{121}$ (Fig. 12).

As observed in previous reports, most of the researchers have followed the hydrothermal approach for the synthesis of quantum dots from various agricultural wastes. The yield and 


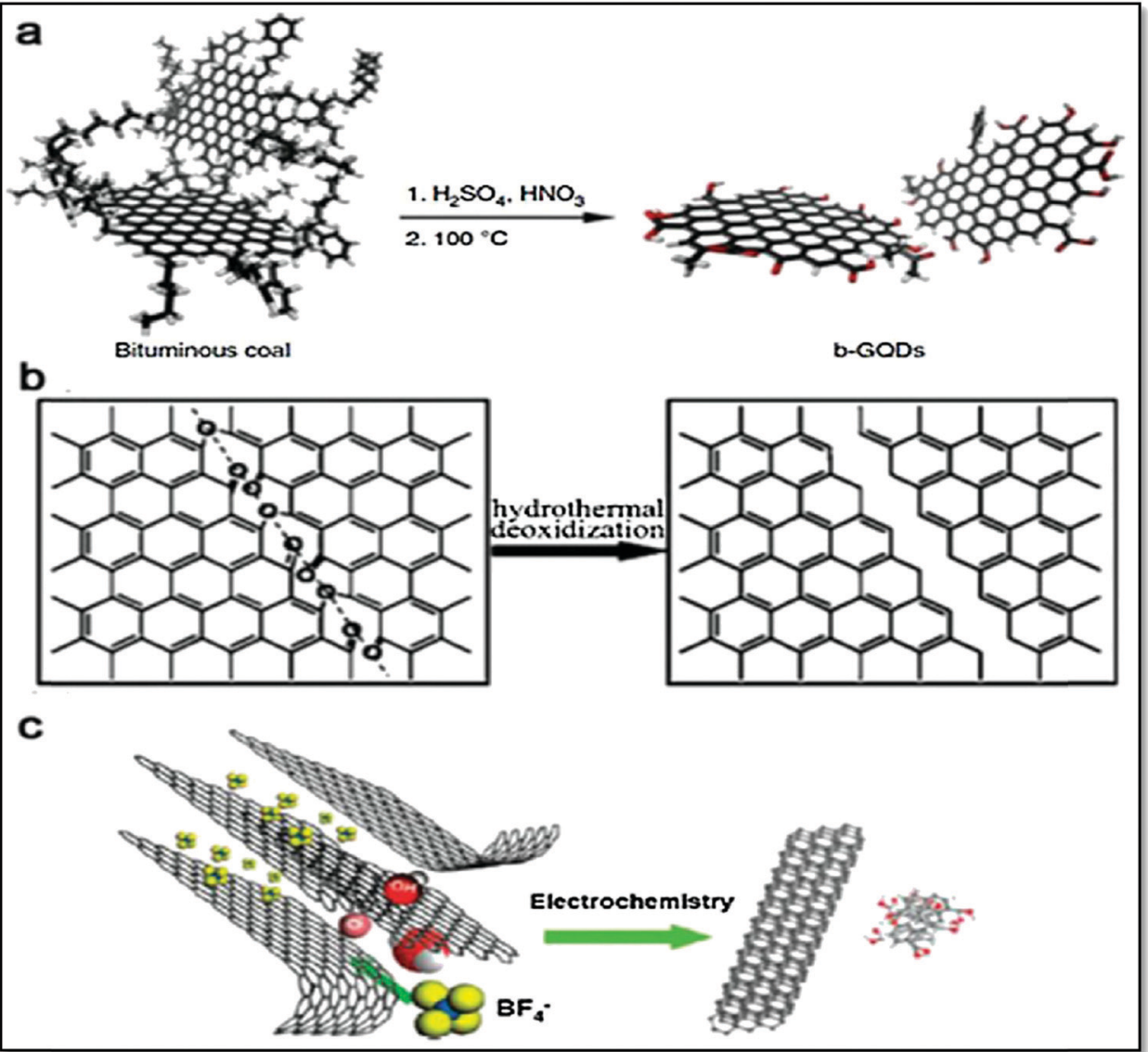

Fig. 12 (a) Microwave synthesis of GQDs, (b) GQDs synthesis by exfoliation of CNTs, (c) GQDs synthesis by exfoliation of graphite. ${ }^{122}$ Reproduced by permission of Elsevier.

properties of the developed CQDs mainly depend on the parameters of the manufacturing method such as centrifugation speed, concentration of solid waste, oxidation time, and temperature of the hydrothermal reactor. The time duration of the hydrothermal reaction also plays a crucial role that affects the synthesis and quality of CQDs. Moreover, the variation of different oxidizing agents and solvents affects the optical properties of the synthesized CQDs. The tabulation of the above reported data on the synthetic procedure and the conditions for the development of different CNMs using solid waste as the precursor has been summarized in Table 2 .

It can be clearly visualized from Table 2 that for the synthesis of graphene and CNTs, usually, pyrolysis and CVD processes are followed, while quantum dots are synthesized via the hydrothermal methodology. The comparison in the structure and properties of CNMs derived from solid wastes and conventional graphite precursors is not dependent on the carbon precursors rather the presence of some impurities in the wastes that create some defects, such as edge dislocation and point dislocation in the case of waste-derived CNMs. ${ }^{86}$ The traditional Hummers' method synthesizes graphene, which usually appears in the oxide form (i.e., graphene oxide), is brownish in colour. On the other hand, pyrolysis and CVD process provide graphene that usually possesses lower oxidative functionality due to high heating rates and pressure. Based on the analysis from the references in Table 2, it is observed that the slow rate of pyrolysis, quantity of the catalysts, and the temperature of annealing are the key factors for the steady conversion of carbonic solid waste materials into CNMs. These observations not only give us a clue about the role of the catalyst for the decomposition of the carbonic layers but also offers a handful of information about the role of high temperature degradations on the formations of graphene. In the CVD based methods, the quality of the CNMs is strongly correlated to the processing temperatures and flow rates of hydrogen/argon. In the CVD growth phase, the optimum rate of gas flow per sccm with a particular time span is necessary for the development of graphene foil (GF). Overall, the quality of graphene mainly depends upon the atomic composition of solid waste materials, amount of catalyst, pyrolytic temperature, and gas flow rates. Graphene obtained by pyrolysis and CVD procedures is mainly obtained in its 2-3 layered form. It is may be due to the process of high temperature exfoliation of carbonic form, which allows van der Waals forces of attractions between the layers of carbon to get distorted; thus, the separation of carbonic layers becomes easier. Furthermore, the excessive presence of heteroatoms, metal ions, and metal oxides can influence the purity of synthesized graphene. 
Table 2 Synthesis methodologies for CNMs using solid waste materials as the precursors

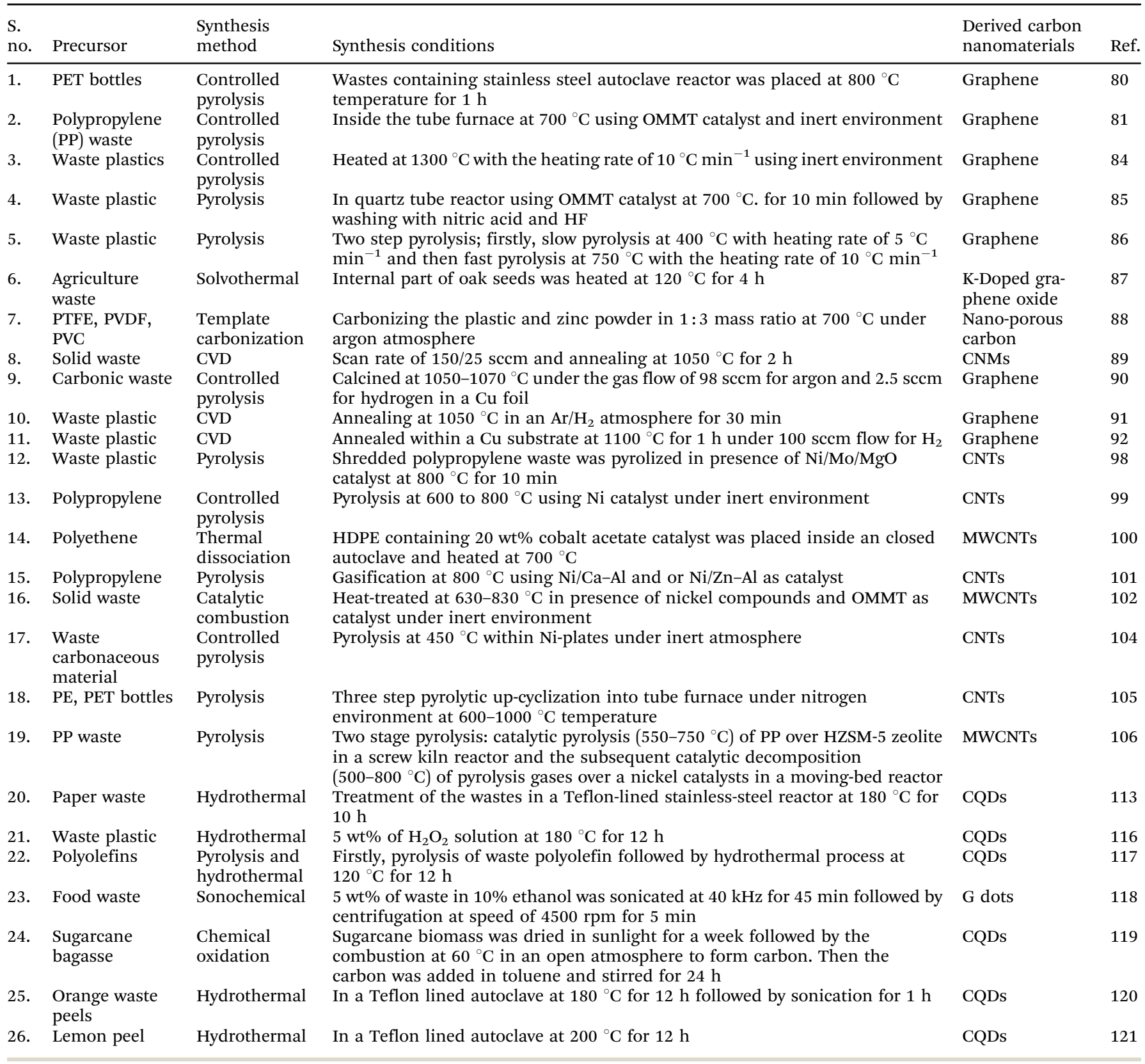

\section{Structural and morphological properties}

Structural properties such as the degree of carbonization or graphitization, pore size, pore volume, and their surface morphologies are strongly correlated to the electrochemical performance of the fabricated device. The doping of metals and heteroatoms can also control the structural and morphological properties to some extent.

\subsection{Degree of carbonization}

The degree of carbonization is very crucial for the electrochemical study of solid waste based CNMs. The degree of carbonization alters the rate capacity, comprehensive resistance, power density, and power delivery along with the cyclic stability and energy efficiency. Carbonization improves the hydrophilicity for aqueous electrolytes, which mainly enhances the diffusion of ions along with the transport number, which enhances the capacitive performance of the fabricated devices. ${ }^{123}$ The low resistivity suggests high conductivity in the sample associated with a high degree of carbonization or carbonic activation.

But as the carbonization and graphitization increase, the relative pore volume and the structure gets distorted due to smaller specific area. Thus, in order to maximize the electrochemical potential, we need to find the compromising link in between the degree of carbonization and the pore structure. High temperature pyrolysis in an inert atmosphere usually reduces the functional moieties of the material, which can 
somehow limit the reach of the exact surface area for the corresponding material. The transition metal and their oxide based catalytic degradation of solid waste materials is reported as one of the efficient methods for considerable carbonization or graphitization for high performance electrochemical studies. Sun et al. derived two-dimensional porous carbon nanosheets having an excellent surface area for electrochemical studies by using coconut shell as the raw material. ${ }^{123}$ Here, $\mathrm{FeCl}_{3}$ and $\mathrm{ZnCl}_{2}$ were used to fix the appropriate ionic composition by using them as degradation and activation catalysts, respectively. Thus, the obtained carbon nanomaterial showed an excellent surface area of $1874 \mathrm{~m}^{2} \mathrm{~g}^{-1}$ and good electrical performance of $268 \mathrm{~F} \mathrm{~g}^{-1}$ at $1 \mathrm{~A} \mathrm{~g}^{-1}$ using $\mathrm{KOH}$ as the electrolyte. Zhang et al. reported carbonization using $\mathrm{K}_{4} \mathrm{Fe}(\mathrm{CN})_{6}$ by the chemical activation of hierarchical carbon nanotubes, which further offered good ionic diffusion and excellent conductivity. ${ }^{124}$ The activated carbonic nanotubes were further loaded with $\mathrm{MnO}_{2}$ and the resultant hybrid supercapacitor showed huge electrochemical capacitance of $550.8 \mathrm{~F} \mathrm{~g}^{-1}$ at a current density of $2 \mathrm{~A} \mathrm{~g}^{-1}$, with $62 \%$ retention at the current density of $50 \mathrm{~A} \mathrm{~g}^{-1}$. Interestingly, $90 \%$ capacitance retention was reported even after 5000 cycles for the same device.

Carbonization at a temperature $1000{ }^{\circ} \mathrm{C}$ or above results in excellent conductivity; thus, the prepared CNMs usually act as excellent candidates for semiconductors, supercapcitor electrodes, and temperature-resistant catalyst carrier. Typically, the conductivity increases up to $1000{ }^{\circ} \mathrm{C}$ temperature due to the relative decrease in the CNMs diameter and increase in the surface area. On the other hand, the favorite alignment of the carbonic layers having a flat surface rises significantly, the cavity volume declines efficiently along with the increase in the crystallite dimension. Consequently, with the increase in the reaction temperature, the CNMs structure commences to change into flake-type during the dehydrogenation reaction. ${ }^{125}$ Higher temperature can significantly increase the conductivity. Thus, the CNMs carbonized at $1000{ }^{\circ} \mathrm{C}$ have excellent 'degree of carbonization'. At higher temperature range, the outflow and volatilization of atoms creates pores on the CNMs surface, which makes these CNMs excellent candidates for different electrochemical applications. The increase in the carbonization temperature leads to a denser structure at the nanoscale with a reduced diameter. This specifies that carbonic weight percentage, in such cases, increases with increasing carbonization temperature, resulting in progressive graphitization at higher temperature. CNMs at higher temperature range show significantly higher conductivity in comparison to CNMs obtained at lower temperature, mostly due to the availability of moving electrons. $^{125}$

\subsection{Porosity \& surface framework}

Solid waste derived CNMs are usually formed with unique structural framework, which include spherical tubes, hierarchical spheres, honeycomb lattice of carbon, carbon onion, and vertical flower. On the other hand, the electrical performance of the supercapacitors usually increases with increasing specific surface area of the active material with some diversions.
In addition to the surface area, the size of the pores, their distribution, and pore volume are also important factors that directly affect the possible electrochemical performance of the device (Fig. 13). The presence of macropores (i.e., size $>50 \mathrm{~nm}$ ) can perform as the storage sites for different metal or nonmetallic ions, whereas mesopores $(2-50 \mathrm{~nm})$ can offer channels for charge transfer. Micropores ensure high specific surface area and contribute to the electrical double layer capacitance. To obtain the optimized performance, researchers are synthesizing hierarchically porous CNMs, which contain both the mesopores and micropores by adjusting the experimental parameters, such as the amount of the actuating reagent, ${ }^{126-128}$ pyrolysis temperature and pressure, ${ }^{129-133}$ and activating reagents. ${ }^{134-139}$

Recently, highly microporous activated carbon was synthesized from cotton stalk by Ma et al. ${ }^{143}$ They altered the mixing proportion of $\mathrm{KOH}$ and carbon and highly ordered, microporous activated carbon (HOMAC) was obtained using the $\mathrm{KOH}$ carbon ratio of $1: 4$. The synthesized microporous material-based supercapacitor device showed good specific capacitance of $322 \mathrm{~F} \mathrm{~g} \mathrm{~g}^{-1}$ along with an energy density of $33.91 \mathrm{~kg}^{-1}$ using $\mathrm{H}_{2} \mathrm{SO}_{4}$ as the liquid electrolyte.

Cui et al. reported the synthesis of porous 3D carbon aerogel by taking proliferate-green-tide in the pyrolysis process, followed by appropriate chemical activation. ${ }^{145}$ The variations in the temperature for the activation process works as the regulator of the resulting pore structure and pore sizes, i.e., macro, meso, and micropores. The obtained material showed an excellent surface area of $2200 \mathrm{~m}^{2} \mathrm{~g}^{-1}$ and a good specific capacitance of $260.6 \mathrm{~F} \mathrm{~g}^{-1}$ in the liquid electrolyte, whereas $92 \%$ retention for 10000 cycles was reported for the same device. They also checked the effects of different activation agents on the porosity and porous nature of the carbonic nanomaterials using $\mathrm{CaCl}_{2}, \mathrm{NaCl}, \mathrm{ZnCl}_{2}, \mathrm{KCl}, \mathrm{K}_{2} \mathrm{FeO}_{4}$, and $\mathrm{H}_{3} \mathrm{PO}_{4}$. Thangavel et al. explained the relationship between the electrochemical performance and the designed structure of the carbonic materials with different activators such as $\mathrm{KOH}$, $\mathrm{ZnCl}_{2}$, and $\mathrm{H}_{3} \mathrm{PO}_{4} \cdot{ }^{146}$ They showed very strong correlation between the activating agents and the structural variations of different pores. The $\mathrm{KOH}$ activated material showed hierarchical, open, and large pores. The pores with $\mathrm{ZnCl}_{2}$ were invisible and the sample activated with $\mathrm{H}_{3} \mathrm{PO}_{4}$ showed pores embedded into different cavities.

Wang et al. synthesized activated porous material from cornstalk biomass, where they used neutral salts $\mathrm{NaCl}$ and $\mathrm{KCl}$ as the activating agents during the process. ${ }^{147}$ Fig. 14 represents the schematic illustration of the whole process. Similarly, sulphur and nitrogen co-doped porous carbon was synthesized using ginkgo leaf waste by Zheng et al. ${ }^{148}$ They believed that chemical activation led to the hierarchical structure of mesoporous/microporous CNMs.

The structural framework of porous carbon can be better described as zero dimensional (0D), one dimensional (1D), two dimensional (2D), and three dimensional (3D) materials. The direct correlation between the pore size and distance of ionic transport is a very crucial factor for the enhancement of the 

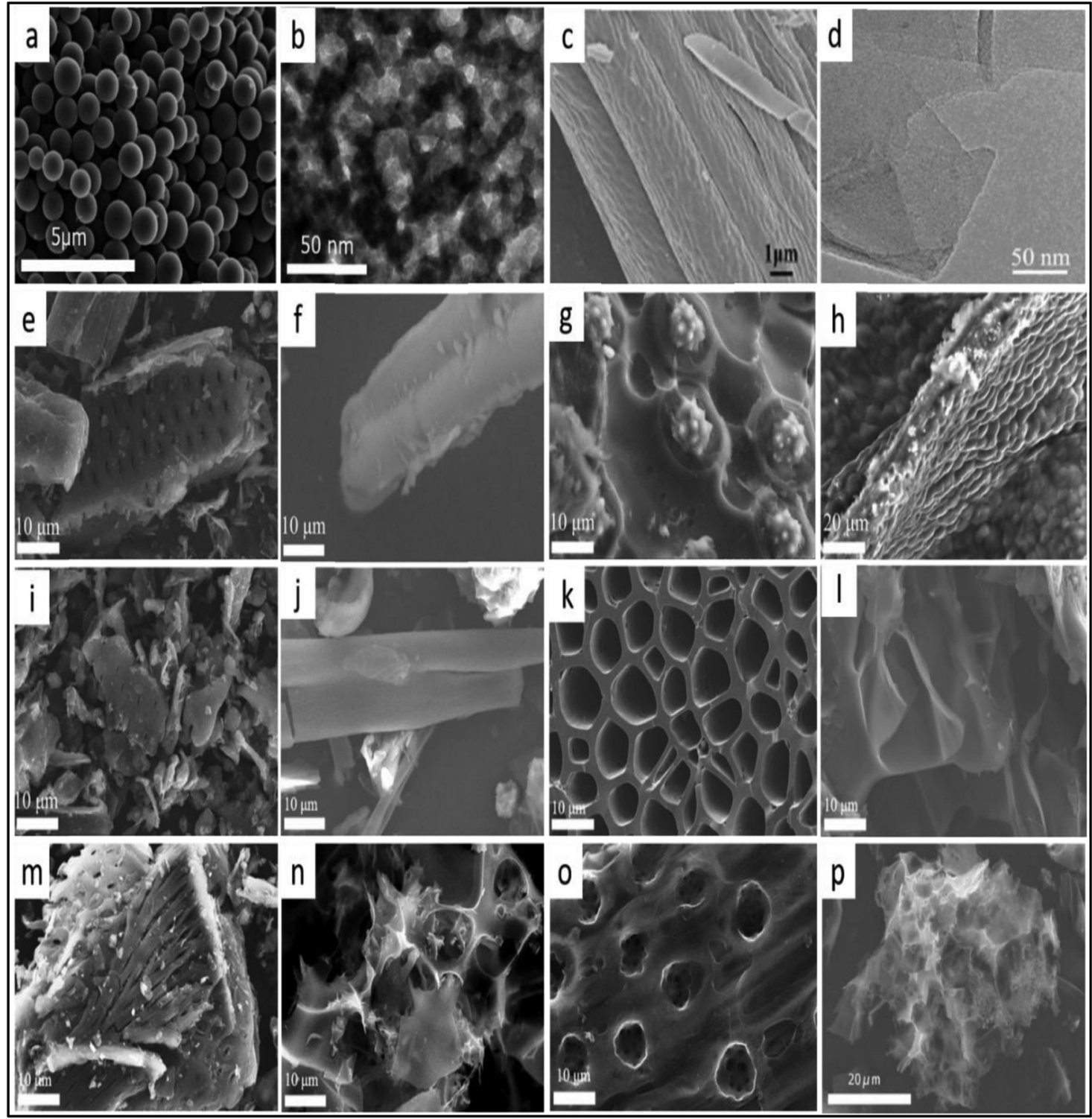

Fig. 13 Electron microscopic images of ( $a$ and $b$ ) fermented rice derived porous carbon spheres, ${ }^{132,140}$ Reproduced by permission of Elsevier and RSC, (c) dandelion derived active carbon, ${ }^{132,141}$ Reproduced by permission of Elsevier, (d) coconut derived porous graphene nanosheets, ${ }^{132,142}$ Reproduced by permission of Elsevier and RSC, (e) cotton stalk, (i) carbonized cotton stalk, (m) activated cotton stalk, ${ }^{132,143}$ Reproduced by permission of Elsevier, (f) Saussurea involucrata stalk, (j) carbonized Saussurea involucrata stalk, (n) activated Saussurea involucrata stalk. SEM images of (g) fresh coconut shell, $(\mathrm{k})$ after the carbonization of the coconut shell and (o) activated coconut shell. The SEM image of algae (h), carbonized algae (l), activated algae (p). ${ }^{132,144}$ Reproduced by permission of Elsevier and RSC.

capacitors' performance. Recently, various structures have been reported for solid-derived CNMs, such as $0 \mathrm{D}$ hollow structure, 1D nanofibers, 1D nanotubes, 2D nanosheets, and 3D hierarchical framework, which are discussed in the section below.

4.2.1. OD carbon nanomaterials. $0 \mathrm{D}$ carbon nanomaterials are popular electrode candidates due to their huge surface area and good charging capacity. The solid biomass waste derived 0D CNMs diameter usually ranges in between 50 and $500 \mathrm{~nm}$. Yan et al. synthesized oat-meal derived OD CNMs using the hydrothermal process following the carbonization approach. They reported a glossy surface containing CNMs having $s$ diameter of $2 \mu \mathrm{m} .{ }^{150}$ Recently, Gaddam et al. synthesized 0D
CNMs using coconut oil as the precursor and the prepared oD CNMs exhibited a diameter in the range from $40 \mathrm{~nm}$ to $50 \mathrm{~nm}$ along with an accessible surface area of $56 \mathrm{~m}^{2} \mathrm{~g}^{-1} \cdot{ }^{151}$ Rufford et al. used sugarcane bagasse as the precursor for the synthesis of 0D CNMs. The materials showed a specific surface area of $2871 \mathrm{~cm}^{2} \mathrm{~g}^{-1}$ and a pore volume of $0.81 \mathrm{~cm}^{3} \mathrm{~g}^{-1}$. During the synthetic step, $\mathrm{ZnCl}_{2}$ was used as the activator for sugarcane bagasse based 0D CNMs. ${ }^{152}$ In contrast to the solid spheres, which do not show good tendency to support charge transport and diffusion of the electrolytic ions during electrochemical performance testing, the hollow spheres have gained extensive interest from the scientific community. 

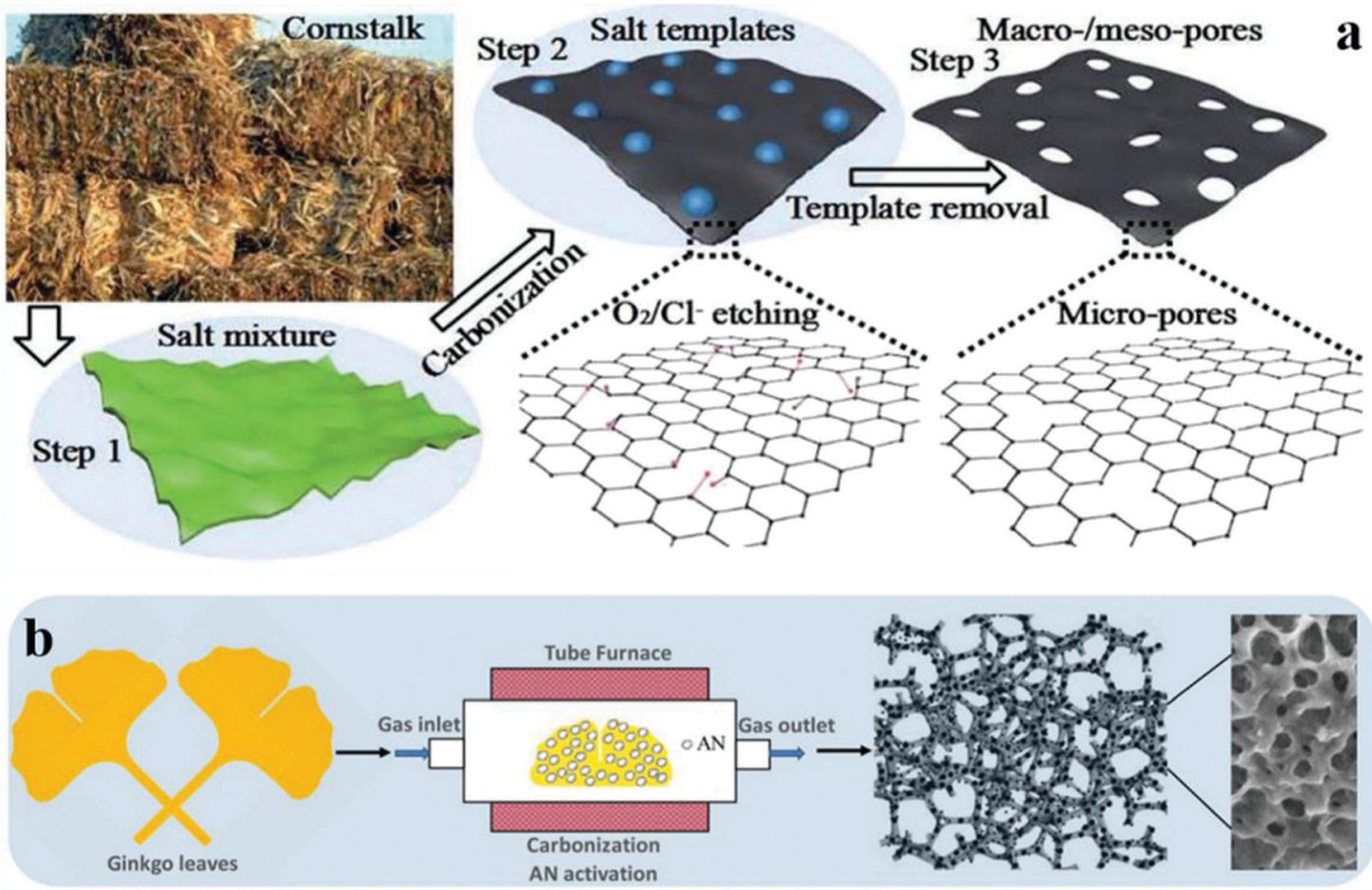

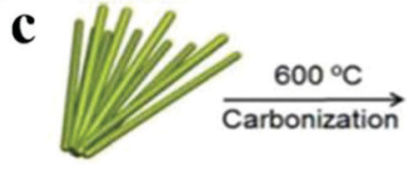

Pine needles

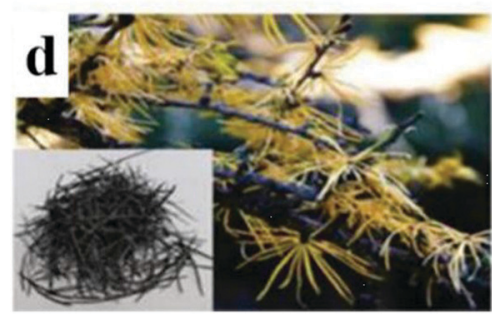

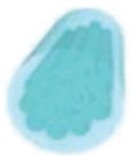
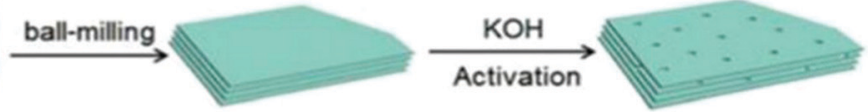

PNCs
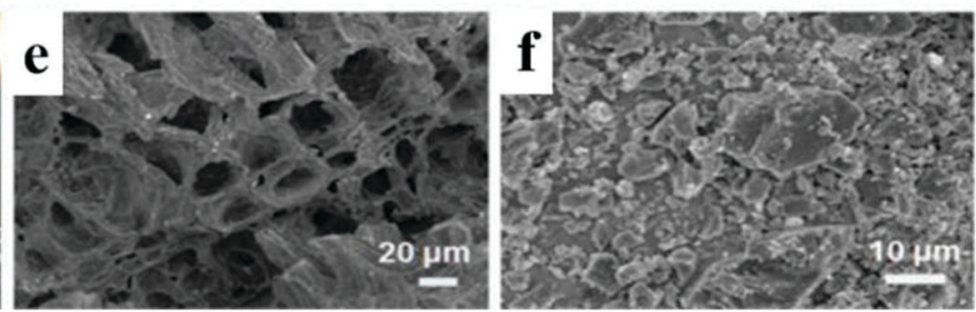

Fig. 14 (a) Synthesis of the meso and macroporous structure from cornstalk, ${ }^{132,147}$ Reproduced by permission of Elsevier and RSC. (b) Synthesis of hierarchically porous carbon from ginkgo leaf, ${ }^{132,148}$ Reproduced by permission of Elsevier and ACS. (c) Synthesis of porous carbon from pine needles. (d) Image of pine needles. SEM images of activation at (e) $600{ }^{\circ} \mathrm{C}$, (f) $900{ }^{\circ} \mathrm{C} .{ }^{132,149}$ Reproduced by permission of Elsevier and RSC.

Gao et al. reported the synthesis of hollow 0D spheres using fermented rice as the precursor. The reported hollow $0 \mathrm{D}$ carbon sphere showed a huge specific surface area of $2106.0 \mathrm{~m}^{2} \mathrm{~g}^{-1}$ along with a pore volume of $1.1 \mathrm{~cm}^{3} \mathrm{~g}^{-1}$. $^{140}$ The special hollow skeleton of carbon is usually obtained by activating agents such as $\mathrm{ZnCl}_{2}$, which usually transfers ions from the electrolyte to the electrode quickly (Fig. 13a and b). The fabricated device showed a good specific capacitance of $219 \mathrm{~F} \mathrm{~g}^{-1}$ at a high current density of $15 \mathrm{~A} \mathrm{~g}^{-1}$. Usually, the interconnected hollow 0D carbon spheres skeleton provides continually open ionic transfer and ionic diffusion at the activated surface area. It accesses and insures good chemical activation, and charging/ discharging during the electrochemical performance testing of the fabricated device.

4.2.2. 1D carbon nanomaterials. One-dimensional CNMs usually differ from $0 \mathrm{D}$ CNMs in having a higher aspect ratio, which somehow benefits them by increasing the relative surface area. Carbon nanotubes and carbon nanofibers are the most utilized 1D materials in electrochemical studies. Electrochemical spinning is the most simplified and easy procedure to synthesize 1D carbon nanofibers. Usually, they have s size in the range of $100 \mathrm{~nm}$. The plant waste (lignin based) was used as the precursor for the synthesis of carbon nanofiber via the electrochemical spinning process by various researchers. In brief, electrochemical spinning consists of a capillary tube having a proper power supply along with a collector device. The solid waste is mixed with reagents to properly design the carbon fibre and then mixed uniformly with the polymeric solution in a capillary tube.

Here, the potential flow and its rate, and the quantity of the solution along with the length of the whole device work as crucial factors to adjust the structure of carbonic nanofibers. 
Carbon fibers were reported with polymer incorporation in most of the studies, especially via the cross-linking framework. Mainly, polymers are used to enhance the viscosity of carbon fibers in the electrochemical spinning methodology. Recently, Tao et al. reported the liquification of walnut shells by phenol along with the addition of polyvinyl alcohol for one hour during the spinning process. ${ }^{153}$ They quoted increased fluidic activity of the solution by the addition of polyvinyl alcohol; the process resulted in the synthesis of $1 \mathrm{D}$ carbon fibers having a diameter of $280 \mathrm{~nm}$. Thus, the obtained walnut shell/PVA nanofiber was further used for supercapacitor device fabrication and showed excellent specific capacitance of $380 \mathrm{~mA} \mathrm{~h} \mathrm{~g}{ }^{-1}$ at $0.03 \mathrm{~A} \mathrm{~g}^{-1}$ with good cyclic stability.

Liu et al. reported the synthesis of $1 \mathrm{D}$ porous nanofiber using lotus seed pods as the precursor through the activated carbonization method. ${ }^{154}$ The fabricated devices showed good energy density of $13 \mathrm{~W} \mathrm{~h} \mathrm{~kg}^{-1}$ and a power density of $260 \mathrm{~W} \mathrm{~kg}^{-1}$ in the two-electrode system. A good precursor solid waste can have a hollow framework as that in cotton and willow catkins for the synthesis of 1D nanofibers. On the basis of various reported articles, cotton fibre could be used as the best candidate for the synthesis of 1D fibers via thermal cracking and the pyrolysis approach. Similarly, willow catkins and poplar are used for the synthesis of porous nanotubes via the pyrolytic approach, whereas raw dandelion produces 1D tubular nanofibers. ${ }^{141}$ The framework can be retained via carbonization, which increases the surface and interaction of ions. Hollow nanotubes with good porosity usually consist of 20 to $50 \mu \mathrm{m}$ structural length, where tubes are found to be interconnected. Overall, both $1 \mathrm{D}$ nanofibers and the nanotubular structure suggest a new route for charge transfer along with minimizing the ionic diffusion during the electrochemical activation process.

4.2.3. 2D carbons nanomaterials. High temperature cracking of solid waste materials into the $2 \mathrm{D}$ framework is an easy, effective, and greener pathway for the promotion of their electrical properties. Different structures open up possibilities of promising ionic transportation and enable $2 \mathrm{D}$ materials as excellent candidates for energy storage devices. Long et al. reported the synthesis of $2 \mathrm{D}$ graphene-shaped structure via the hydrothermal process, followed by pyrolysis. ${ }^{155}$ The excellent surface area of $1100 \mathrm{~m}^{2} \mathrm{~g}^{-1}$ and mass density of $0.96 \mathrm{~g} \mathrm{~cm}^{-3}$ was reported for the $2 \mathrm{D}$ graphene-shaped structure. The synthesized material showed an excellent specific capacitance of $360 \mathrm{~F} \mathrm{~cm}^{-3}$ along with $99 \%$ retention over 10000 cycles. The fabricated device

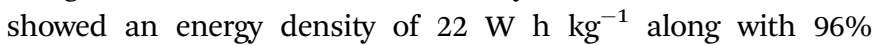
capacitance retention for 10000 cycles. Cornstalk waste biomass based activated carbon nanomaterials were prepared by using $\mathrm{K}_{4}\left[\mathrm{Fe}(\mathrm{CN})_{6}\right]$ as a catalyst during pyrolysis. ${ }^{156}$ Wang et al. used hemp fibers to design the synthesis of graphene-shaped $2 \mathrm{D}$ nanomaterial. The diameter of hemp fibers was in between $10 \mathrm{~nm}$ to $30 \mathrm{~nm}$ and is specially made up of multi-level cellulose and lignin framework. The hemp usually has inherent landscapes of being incorporated internally in 2 dimensions, which can be further utilized to be linked with different materials via activation and carbonization. Zheng et al. reported the synthesis of ultrathin 2D CNM for electrochemical performance testing taking glucose as the precursor material. ${ }^{157} \mathrm{KOH}$ was used for the chemical activation of the synthesized $2 \mathrm{D}$ nanomaterial. Thus, the fabricated device showed excellent capacitance of $257 \mathrm{~F} \mathrm{~g}^{-1}$ at $0.5 \mathrm{~A} \mathrm{~g}^{-1}$ with $72 \%$ retention of capacitance at a current density of $100 \mathrm{~A} \mathrm{~g}^{-1}$. The good percentage of micropores and mesopores produces a huge surface area of $2600 \mathrm{~m}^{2} \mathrm{~g}^{-1}$. Thus, the obtained material is clearly responsible for efficient high electrochemical performance of the fabricated device. Usually, the layers alignment and their incorporation in the metal ions is responsible for fastening of ionic diffusion, which results in an enhancement in electrochemical capacitance.

4.2.4. 3D porous and hierarchical carbon nanomaterials. The excellent capacitance performance, along with good energy density and high-power density of the supercapacitor device, have been achieved by good charge accumulation through the surface contact in between electrolyte and electrode. But lower energy density of the electrode material is associated with the disadvantage of supercapacitors. Due to high conductivity, electrochemical, and thermal stability, the hierarchical carbonic nanomaterials have been identified as crucial candidates to deal with lower energy density. However, the power density of porous carbon can be degraded by pore blockage, which affects the availability of ions under higher current density.

Feng and group reported the unique synthesis of polyporous 3D carbonic structure by using waste bagasse as the precursor for hydrothermal method, followed by subsequent chemical activation. The huge surface area and good porosity behavior is reported for porous 3D framework that showed a surface area of $2296 \mathrm{~m}^{2} \mathrm{~g}^{-1}$. The fabricated device showed a specific capacitance of $320 \mathrm{~F} \mathrm{~g}^{-1}$ at $0.5 \mathrm{~A} \mathrm{~g}^{-1}$ with $71 \%$ retention of capacitance at $50 \mathrm{~A} \mathrm{~g}^{-1}$. Recently, Hao et al. synthesized 3D CNMs using bagasse as the raw material. ${ }^{158}$ The fabricated supercapacitor device delivered good specific capacitance of $142 \mathrm{~F} \mathrm{~g}^{-1}$ at $0.5 \mathrm{~A} \mathrm{~g}^{-1}$. The prepared device showed $94 \%$ retention of capacitance over 5000 cycles. Similarly, Fan et al. reported the synthesis of honeycomb-shaped CNMs using wheat flour waste as the raw material via pyrolysis approach along with chemical activation by $\mathrm{KOH} .{ }^{159} 3 \mathrm{D}$ hierarchical and porous framework produced a large surface area of $1313 \mathrm{~m}^{2} \mathrm{~g}^{-1}$ and displayed excellent specific capacitance of $473 \mathrm{~F} \mathrm{~g}^{-1}$ at $0.5 \mathrm{~A} \mathrm{~g}^{-1}$ using $6 \mathrm{M} \mathrm{KOH}$ as the liquid electrolyte. Recently, activated carbon was derived from cotton stalk, Saussurea involucrata stalk, coconut shell, and algae, and is depicted in Fig. 13e-h.

The pores in the structure of cotton stalks were found to have a diameter of $2 \mu \mathrm{m}$, while the stalks of Saussurea involucrata have a high aspect ratio and the coconut shell showed long fibrous and denser particle distribution on the surface. Clear changes in their structural morphologies have been observed after carbonization (Fig. 13i-l). ${ }^{142}$ The SEM images of the CNMs after the activation process are shown in Fig. 13m-p. Fig. 13m depicts the presence of clear vacancies in cotton stalk derived 3D CNMs. ${ }^{142}$ Similarly, CNMs derived from Saussurea stalk displays a distinctive hierarchical porous carbonic structure. The activated carbon obtained from cotton stalk showed the capacitance of $254 \mathrm{~F} \mathrm{~g}^{-1}$ at a current density of $0.2 \mathrm{~A} \mathrm{~g}^{-1}$, in comparison to that of the hierarchical porous carbon nanomaterial synthesized from 
Saussure stalk, which displayed a specific capacitance of $322 \mathrm{~F} \mathrm{~g}^{-1}$ at $0.2 \mathrm{~A} \mathrm{~g}^{-1}$ current density. The CNMs derived from coconut shell displayed an electrochemical capacitance of $157 \mathrm{~F} \mathrm{~g}^{-1}$ at a current density of $0.2 \mathrm{~A} \mathrm{~g}^{-1}$. It also showed $100 \%$ retention of capacitance over 10000 cycles at the current density of $1 \mathrm{~A} \mathrm{~g}^{-1}$. The hierarchical porous carbonic network is crucial for ionic diffusion and transportation along with charge storage. The electrochemical capacitance of $283 \mathrm{~F} \mathrm{~g}^{-1}$ was reported for the hierarchical 3D porous network at a current density of $0.2 \mathrm{~A} \mathrm{~g}^{-1}$. These results indicate the advantages of porous fibrous CNMs for utilization in supercapacitor application.

The internal framework is usually interrelated with the intrinsic structure of the precursor materials. For example, cotton stalk-derived porous CNM exhibits similar morphological features (Fig. 13e and $\mathrm{m}$ ). ${ }^{142}$ Table 3 depicts the variations in the pore sizes of different solid waste derived materials and the cyclic stability of the respective capacitor device.

The mesoporous nanomaterials usually have a cylindrical pore surface and a wormhole-like structure. Based on this, it can be said that ion transportation usually takes place through the pores in electric double layer (EDLC) capacitors. But because of the very smaller pore size of the micropores, the electrolyte ions can form an electrical wire in the cylindrical capacitor (EWCC) network. ${ }^{169}$ The electrochemical transportation of ions for the process of charging and discharging is mainly dependent upon the relative pore size, pore shape, and their distribution. ${ }^{170}$ The hierarchical and porous carbonic network in $0 \mathrm{D}, 1 \mathrm{D}, 2 \mathrm{D}$, and three-dimensional lattice usually tend to shorten the diffusion distance for ions travelling from electrolyte to electrodes and ultimately minimizes the diffusion time.

However, the control and selectivity of CNMs synthesis is especially dependent on the processing temperature, pressure and catalyst used during the process (pyrolysis or CVD). For the synthesis of graphene, degradation catalyst such as OMMT and ZSM are specially used due to their efficiency for CNMs production, ease of removal, and lesser consumption during the processing and manufacturing of graphene. However, during the synthesis of CNTs, catalysts, especially nickel, aluminum, cobalt, and other transition metal ions are used owing to their tendency of easy electron liberation during the high temperature exfoliation process, which might intercalate into the

Table 3 Precursors of various carbonic bio-waste used for the synthesis of CNMs, their pore volumes, and cyclic stability of the corresponding capacitor devices

\begin{tabular}{lllrr}
\hline & $\begin{array}{l}\text { Carbonic bio-waste } \\
\text { S. no. }\end{array}$ & $\begin{array}{l}\text { Pore volume of the precursor } \\
\text { CNMs }\left(\mathrm{cm}^{3} \mathrm{G}^{-1}\right)\end{array}$ & $\begin{array}{l}\text { Stability } \\
\text { (cycle Life) }\end{array}$ & Ref. \\
\hline 1. & Acacia based gum & 0.99 & 1000 & 159 \\
2. & Sugarcane bio-waste & 0.81 & 10000 & 160 \\
3. & Flax & - & 1000 & 161 \\
4. & Bio-waste of corncob & 1.95 & 100000 & 162 \\
5. & Beans of coffee & 0.57 & 2000 & 163 \\
6. & Coconut outer shell & 1.21 & 2000 & 164 \\
7. & Rice plants husk & 1.07 & 1000 & 165 \\
8. & Oil Palm & 0.02 & 2500 & 166 \\
9. & Carrageenan & 1.43 & 1000 & 167 \\
10. & Shaddok peelings & 0.49 & 132 & 168
\end{tabular}

cluster of the carbonic layers, resulting in the easy exfoliation of the carbonic layers.

\subsection{Surface properties}

Precisely, the surface area, porous structure, and other morphological features signify the electrochemical potential of solid waste derived CNMs, while the porous nature of activated CNMs can be easily monitored via chemical doping and activating by different methodologies that somehow modulates the variations in the porous nature, viz., meso, micro, and macro. On the other hand, thermal heating, as well as chemical and acidic treatments can adjust the position and performances of the surface functional groups. The adjustment of the functional moieties does alter many morphological features of solid waste derived materials, which commonly include electrical and mechanical properties. ${ }^{132,171}$

The doping of various metallic and non-metallic elements such as transition metals, p-block gases, and heavy metals usually signifies the surface porosity and surface morphology including surface area and electrochemical conductance of the agricultural waste based carbonic nano-materials. ${ }^{172}$ Recently, researchers are focusing on the synthesis of CNMs by using solid waste materials as the precursors and their further doping via heteroatoms to obtain a visible separation in between the layers of synthesized CNMs or to intercalate the metal ions for obtaining a more acute porous nature for the enhancement of electrochemical properties. ${ }^{132,171}$ Zhang et al. synthesized oxygen-containing carbon nanomaterials using biological gelatin as the precursor, having a layered morphology. ${ }^{172}$ The synthesized carbonic clustered nanomaterial showed excellent electrochemical specific capacitance of $276.6 \mathrm{~F} \mathrm{~g} \mathrm{~g}^{-1}$ at current density of $1 \mathrm{~A} \mathrm{~g}^{-1}$. The electrochemical performance remained intact and retained almost $73 \%$ of the capacitance at $100 \mathrm{~A} \mathrm{~g}^{-1}$. They outlined the efficiency of oxygen as the modulator for the electrochemical properties. ${ }^{172}$ Similarly, nitrogen is another heteroatom that is extensively studied for the device fabrication of various electrochemical studies. The Doping of nitrogen is reported to increase the conductivity of carbon nanomaterials and enhances the specific capacitance of the resulting materialbased supercapacitor device. This may be due to the presence of lone pair of electrons in the nitrogen atom, which is available for bonding and conduction purpose, thus increasing the conductivity and electrochemical performance of the resulting CNMs. Nitrogen usually exists in three different structural carbonic species, i.e., pyridine nitrogen, pyrrole nitrogen, and quaternary nitrogen. The ring atoms of pyridine nitrogen are reported to exist in $\mathrm{sp}^{2}$ hybridization and the lone pair of electrons, due to the aromatic nature of ring, projects themselves outside the ring. This enhances the electrochemical properties of the doped carbonic materials. On the other hand, the pyrrole nitrogen ring has no aromatic character and the lone pairs are delocalized, which also manage to enhance the conductive property of the doped carbon nanomaterials. On the other hand, the quaternary nitrogen atom can only enhance the conductivity by the ionization of the carbonic electrode. ${ }^{171}$ Recently, Hou et al. reported the synthesis of hierarchically thin 
activated carbon nanomaterial using raw silk as the precursor, where the chemical activation and carbonization procedures was followed thoroughly for the enhancement of electrochemical conductivity and the performance of the electrode material. ${ }^{173}$ A nitrogen rich mesoporous carbonic cluster having a huge specific surface area of $1030 \mathrm{~m}^{2} \mathrm{~g}^{-1}$ was synthesized by Zhou et al., which showed $15.0 \mathrm{~nm}$ diameter of pore size along with $7 \mathrm{wt} \%$ of nitrogen as the doped atom. The synthesized material showed excellent specific capacitance of $264 \mathrm{~F} \mathrm{~g}^{-1} .^{174}$ Cellulosic precursor based porous carbon material was synthesized by Lu et al. They reported microcrystalline structured CNM, along with intrinsic nitrogen doping. ${ }^{175}$ The hierarchical porous structure along with high nitrogen content is more responsible for charge storage via pseudo storage mechanism. Thus, the synthesized material showed exceptional electrochemical capacitance of $426 \mathrm{~F} \mathrm{~g}^{-1}$ with excellent cyclic stability. ${ }^{175}$ 3D oxygen and nitrogen doped carbon nanosheets were reported by Liu et al. by taking waste wood as the precursor. They followed the methodology of chemical oxidation, bio-swelling, and low temperature pyrolysis for the synthesis of the material. ${ }^{176}$

Similarly, some other heteroatoms such as phosphorous, boron, and sulphur are also reported as electrochemical performance booster for CNMs by enhancing their surface area and adding the pseudo capacitive property. Ling et al. reported a flexible device with co-doping of boron and nitrogen through annealing process. ${ }^{177}$ Here, boric acid was used as the source of boron doping. The fabricated device showed excellent specific capacitance of $268 \mathrm{~F} \mathrm{~g}^{-1}$ at $0.1 \mathrm{~A} \mathrm{~g}^{-1}$ along with an extended lifetime 10 000-15 000 cycles and 105-113\% capacity retention. Dandelion fluff derived porous and hierarchical carbon nanotubes with good composition of boron and nitrogen was reported by Zhao et al. ${ }^{178}$ The excellent ionic diffusion and fast charge transfer mechanism along with a very stable framework of electrons was reported for the fabricated device. The hierarchical CNTs showed good energy density of $12.2 \mathrm{~W} \mathrm{~h} \mathrm{~L}^{-1}$ and excellent power density of $699.8 \mathrm{~W} \mathrm{~L}^{-1}$. Huang et al. reported phosphorous-doped porous carbon nanomaterial using coffee waste materials as the precursor. The chemical activation of CNM was done with the help of $\mathrm{H}_{3} \mathrm{PO}_{4} \cdot{ }^{179}$ Electrochemical performance testing was performed by using $6 \mathrm{M} \mathrm{KOH}$ as the electrolyte for the process. The energy density of $15 \mathrm{~W} \mathrm{~h} \mathrm{~kg} \mathrm{~kg}^{-1}$ was reported at a good power density of $75 \mathrm{~W} \mathrm{~kg}^{-1}$. A sulphur and nitrogen co-doped hierarchical and porous activated carbon-based device was fabricated by Xu et al., using amino acid rich bean shells along with some vitamins as precursor material. ${ }^{180}$ The bean was pyrolyzed at a high temperature, i.e., at $800{ }^{\circ} \mathrm{C}$ for $2 \mathrm{~h}$ and then activated via organic reaction at high temperature along with an inert atmosphere of nitrogen with a constant heating rate of $3{ }^{\circ} \mathrm{C} \mathrm{min}^{-1}$. They reported that sulphur enhances the space through the exclusive sorption of ions, whereas nitrogen enhances the electrical conductivity and wettability for the fabricated electrode. The specific electrochemical capacitance of $202 \mathrm{~F} \mathrm{~g}^{-1}$ at $0.5 \mathrm{~A} \mathrm{~g}^{-1}$ current density was reported by taking $6 \mathrm{M} \mathrm{KOH}$ as the electrolyte. The heteroatoms used in all the above reports are either directly derived from parts of the solid waste materials or incorporated by any activation method. These evaluations concluded that heteroatom doping in CNMs usually enhances the electrochemical performance of the device.

\section{Effects of CNMs structure on electrochemical properties}

Carbon nanomaterials have become distinct materials in the field of science and technology so far due to their extraordinary properties starting from the electrical, mechanical, and thermal properties. ${ }^{181-184}$ On the other hand, some properties such as chemical stability, light weighted structure, excellent optical transparency, and extraordinary mechanical flexibility are responding good to the new technologies in various respective fields, such as in energy application, i.e., solar cells, supercapacitors, fuel cells, Li-ion batteries, and in the biomedical field, i.e., drug delivery, bioimaging, and biosensors. ${ }^{185-190}$ The carbon nanomaterials such as graphene, CQD, and CNT have been synthesized by various research groups, while using expanded graphite in various solid waste materials as starting raw materials. The variations in their physiochemical and mechanical properties have some extraordinary effects in addition. So, for much a deeper understanding of their potential use, it becomes very important to discuss their electrochemical properties in detail.

The solid waste derived carbon nano-materials (CNMs) have vast variations in their electrochemical properties especially due to the variation in the specific surface area and the morphological behavior, while most of the structural properties of CNMs vary along with the variation in the structural framework of the precursor solid waste materials. Morphological expansion and changes in the carbonic framework result in the variation of the electronic conduction inside the layers of graphitic component, which is an appropriate reason behind the difference in the qualitative and conductometric behavior of various carbon nano-materials, such as graphene, CNT, and GQDs.

Lian et al. fabricated a capacitor using polyethylene derived hierarchical porous carbon nanomaterials with the incorporation of ammonia. The obtained activated hierarchical porous carbonic framework was reported with exceptional high surface area and contributes in subsequent capacitance along with a

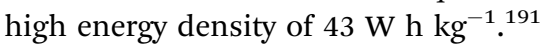

Chen et al. reported porous carbonic material by using straw cellulose waste as the precursor for supercapacitor applications. Thus, the obtained porous carbonic material was activated by sodium hydroxide and showed an excellent rate performance of $281.32 \mathrm{~F} \mathrm{~g}^{-1}$ using $6 \mathrm{M} \mathrm{KOH}$ as the electrolyte at $0.5 \mathrm{~A} \mathrm{~g}^{-1}$. The fabricated device showed $92.93 \%$ cyclic stability with 20000 cycles rate at $1 \mathrm{~A} \mathrm{~g}^{-1}$ and an energy density of $8.67 \mathrm{~W} \mathrm{~h} \mathrm{~kg}^{-1}$ along with a power density of $3.50 \mathrm{~kW} \mathrm{~kg}^{-1}$ using $6 \mathrm{M} \mathrm{KOH}$ solution as an electrolyte. $^{192}$

Recently, Peng et al. derived porous nitrogen doped carbon nanosheets using waste pomelo mesocarps as the precursor along with simultaneous activation and carbonization. The developed 
material showed excellent surface area of $974.6 \mathrm{~m}^{2} \mathrm{~g}^{-1}$ and the fabricated supercapacitor device showed excellent specific capacitance of $245 \mathrm{~F} \mathrm{~g}^{-1}$ at $0.5 \mathrm{~A} \mathrm{~g}^{-1}$ in $2 \mathrm{~mol} \mathrm{~L}^{-1} \mathrm{KOH}$ electrolyte. Moreover, the supercapacitor possesses an energy density of 14.7 $\mathrm{W} \mathrm{h} \mathrm{kg}^{-1}$ at $90 \mathrm{~W} \mathrm{~kg}^{-1}$ power density, which was operated in the potential range of $1.8 \mathrm{~V}$ in aqueous $\mathrm{Na}_{2} \mathrm{SO}_{4}$ electrolyte. ${ }^{193}$ Li et al. reported mangosteen peel derived 3D porous carbonic structure, where they concluded its excellent $\mathrm{CO}_{2}$ absorbing capacity with high specific capacitance. ${ }^{194}$ They reported an increased surface area, i.e., $1270 \mathrm{~m}^{2} \mathrm{~g}^{-1}$ by following hydrothermal methodology in alkaline solution, which showed the excellent capacity of $\mathrm{CO}_{2}$ capture 6.93 and $4.77 \mathrm{mmol} \mathrm{g}^{-1}$ at $0{ }^{\circ} \mathrm{C}$ and $25{ }^{\circ} \mathrm{C}$, respectively. ${ }^{194}$ At last, the electrochemical performance testing of the material showed the capacitance of $240 \mathrm{~F} \mathrm{~g}^{-1}$ using $6 \mathrm{M} \mathrm{KOH}$ in $1 \mathrm{~A} \mathrm{~g}^{-1}$ solution. $^{194}$

Zhang et al. fabricated a supercapacitor device using hierarchically microporous carbonic material on nickel foam. They reported the high specific capacitance of $1601.6 \mathrm{~F} \mathrm{~g}^{-1}$ at $2 \mathrm{~A} \mathrm{~g}^{-1}$ current density for a capacitor with a specific molar ratio, i.e., $4: 1$ of nickel and cobalt. ${ }^{195}$

Recently, W. Xing et al. developed hierarchical porous carbons material for an ultra-capacitor electrode. The chemical activation of the material resulted in the variation in its pore size and thus the reported capacitance was calculated to be about $180 \mathrm{~F} \mathrm{~g}^{-1}$ at $1 \mathrm{~Hz}^{196}$

The electrochemical device was fabricated using biomass waste derived carbon nanomaterials for symmetrical supercapacitors by Deng et al. ${ }^{197}$ They reported doping with multiple heteroatoms with a relative increase in the surface area along with the hierarchical porosity. They also reported the excellent specific capacitance of $412 \mathrm{~F} \mathrm{~g}^{-1}$ in $6 \mathrm{M} \mathrm{KOH}$ electrolyte using a current density of $0.5 \mathrm{~A} \mathrm{~g}^{-1}$. Energy density up to $28.78 \mathrm{~W} \mathrm{~h} \mathrm{~kg}^{-1}$ and the power density of $300 \mathrm{~W} \mathrm{~kg}^{-1}$ at the voltage of $2.0 \mathrm{~V}$ in $1 \mathrm{M}$ $\mathrm{Na}_{2} \mathrm{SO}_{4}$ as an electrolyte was reported along with the cycling stability of 10000 cycles and $92.9 \%$ capacitance retention for the same. $^{197}$

The doping of non-metals, especially p-block elements into carbonic nanomaterials, usually increases the specific charge holding capacity along with electron donation ability of the surface, which somehow alters the electrochemical to thermal properties related to carbonic nanomaterials. The variations in the specific capacitance along with the energy and power density of different electrode materials is mostly due to the alteration in the surface area and the surface behavior of carbon nanomaterials.

Some structural modifications such as hierarchical structure, porous surface, 3D porous modifications, and doping by various different organic and inorganic reagents including metals and non-metals have increased the chances of conduction of electrons within the layer of carbonic framework. This has become a new route to enhance the electrochemical performance of the supercapacitors using 3D porous CNMs as the electrode material.

Hierarchical porous CNMs incorporating heteroatoms such as nitrogen and sulphur possess exceptionally high surface area and high energy density due to the availability of adequate sites for electron capture and release during the redox reaction or double layer formation. ${ }^{190}$ However, the porous carbon materials doped by alkali metal ions such as sodium and potassium also possess higher capacitance, basically due to the ease of electron conduction for charging and charge storage processes. Alkali metals are electron donors, which make them viable donors. In addition, the porous nature of carbonic materials helps in the easy capture of electrons because of the available capture sites developed due to the good porosity. ${ }^{192,193}$ The hierarchical, porous, and 3D CNMs, especially those deposited on metal foam (such as nickel, aluminum, or cobalt), hold huge specific capacitance and energy density, may be due to the ease of ionic movability during the formation of the double layer and during the redox reactions. Moreover, this discussion concludes that the hierarchically porous surface and 3D porous CNMs show are almost similar in specific capacitance; however, the reasons for their charge storage mechanics lies in the dispensation of electrons around the web of carbonic layers, totally depending upon the ease of electron availability, surface area, movability of ions, and the formation of a double layer.

When considering the synthetic methods for CNMs, the desired structure and properties are largely dependent upon the size, shape, foreign element doping, and surface functional groups of the CNMs. Although the properties of the synthesized CNMs does not much dependent on the carbon precursors, however, the presence of foreign elements/compounds during the synthetic procedure could help in the doping and surface functionalities, which strongly influenced the properties of the CNMS. The CNMs derived from solid waste can have metals or phytochemical as impurities, which actually help in modifying their properties. However, some undesired contamination in the CNMs might also decrease their performances compared to purer one. ${ }^{198}$ Among solid waste materials, plastics are loaded with fillers, pigments, metals, agriculture, and food waste, mostly consisting of natural pigments doped with different metal depending on their natural precursors. ${ }^{198}$ These impurities could stay on the developed CNMs and may positively or negatively affect the electrochemical performances of the developed materials.

\section{Solid waste derived CNMs in supercapacitors}

As observed in the above studies, the properties of solid waste derived CNMs are strongly dependent on the raw material and experimental conditions. They show outstanding electrical conductivity and very high specific surface area, which facilitate their application as supercapacitor device fabrication materials. Supercapacitors are classified as electrochemical storage devices, which can store and deliver energy at a very faster rate and offer very high current in a short period. Henceforth, they are frequently applied in electric vehicles, uninterruptible power supplies (UPS), and memory backups in IT systems and in various other fields. An overview of carbon materials (CNMs) utilized in different types of supercapacitors is given in Fig. 15. 


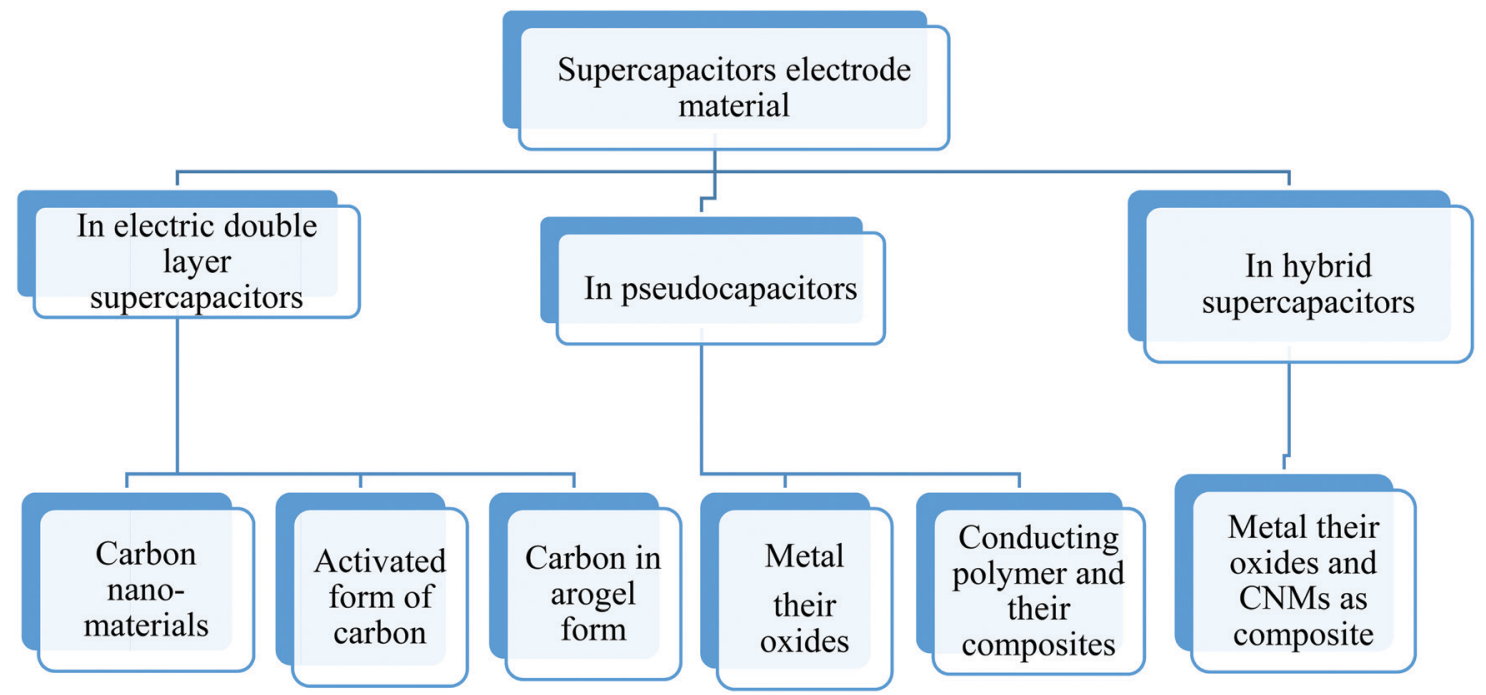

Fig. 15 Hierarchy showing the use of CNMs in different types of supercapacitors.

Different surface structure, variation in the quality of CNMs, i.e., thermo-chemical stability, structural and chemical properties, etc., are the main parameters behind the variations in the electrochemical properties. These materials can be further used as catalytic surfaces for electrode development materials. Graphene itself has an outstanding electrical conductance to charge mobility $\left(>200000 \mathrm{~cm}^{2} \mathrm{~V}^{-1} \mathrm{~s}^{-1}\right),{ }^{199-201}$ reactive chemical inertness, ${ }^{200}$ and also excellent mechanical strength $(>1 \mathrm{TPa}){ }^{202}$ However, the use of CNMs in various energy applications have been reported by various groups, such as electrode materials, catalytic materials for fuel cells, active layer for solar cells, and Li-ion batteries.

The enormously high surface area of CNMs plays a crucial role in the conductivity, capacitance, and storage properties. The conductivity behavior of CNMs and the diffusion rates of the ions are mostly the responsible factors for the storage quality of ultracapacitors. Material development part for supercapacitors is mainly composed of two parts, the first one of carbon nano-materials, while the other part includes different metals, metal oxides, and their polymer composites. Table 4 depicts the various types of supercapacitors and materials used for device fabrication.

Zequine et $a .^{203}$ have reported the flexible supercapacitor device based on bamboo fibers for high temperature supercapacitor applications. They have developed high-performance flexible supercapacitors electrode from carbonized bamboo fibers with specific capacitances of $510 \mathrm{~F} \mathrm{~g}^{-1}$ at $0.4 \mathrm{~A} \mathrm{~g}^{-1}$ with excellent energy density of $54 \mathrm{~W} \mathrm{~h} \mathrm{~kg}{ }^{-1}$. The fibers displayed excellent charge storage stability without degradation up to 5000 charge-discharge cycles. The fabricated supercapacitor device was reported to have an aerial capacitance of $1.55 \mathrm{~F} \mathrm{~cm}^{-2}$ at room temperature. On bending, the fabricated electrode device showed an excellent charge storage capacity with $65 \%$ improvement in the performance at $70{ }^{\circ} \mathrm{C}$ compared to that at $10{ }^{\circ} \mathrm{C} .{ }^{203}$

Mishra et $a .^{203}$ used polypropylene plastic waste for the synthesis of MWCNTs at $800{ }^{\circ} \mathrm{C}$ by chemical vapor deposition
(CVD) in the presence of $\mathrm{Ni}$ as the catalyst. The devices showed the specific capacitance of $59 \mathrm{~F} \mathrm{~g}^{-1}$ with a scan rate of $5 \mathrm{mV} \mathrm{s}^{-1}$ in $1 \mathrm{~N} \mathrm{KOH}$ solution. ${ }^{204}$

According to Zhi et al., ${ }^{205}$ activated carbon for supercapacitor applications can be obtained from petroleum-based precursors, which is prepared by the pyrolysis of raw materials along with chemical activation. Statistical multiple linear regression and stepwise regression methods have been employed to investigate the dependence of specific capacitance on the porosity of activated carbon. Zhi et al. reported that the specific capacitance is dependent on the micropores and their volume while it is independent of the mesopore volume. Nonetheless, the volume ratio of mesopore/micropore dominates over the absolute volume of the mesopores. ${ }^{206}$

Waste polystyrene derived 3D porous CNMs were synthesized via Friedel-Crafts reaction using polystyrene waste as the carbon source. It is believed that high cross linkage and excessive bonding between the carbonyl group and linear polystyrenes provides a good pathway for building a hierarchical porous morphology. Thus, the obtained 3D porous structure showed a large specific surface area along with high capacitance and excellent thermo-chemical stability for ultracapacitors. ${ }^{204}$ On the other hand, waste paper based flexible supercapacitors with their design, applications, and fabrication have been reviewed by Zhang et al. ${ }^{207}$

According to Wen et $a{ }^{208}{ }^{208}$ nano-sized carbon black having nickel oxide in the form of $\mathrm{Ni}_{2} \mathrm{O}_{3}$ works as a brilliant candidate for synergistically catalyzing the carbonization of waste polyolefin for supercapacitor applications. Polypropylene (PP), polyethylene (PE), and polystyrene (PS) were dehydrogenated into CNTs by using carbon black along with nickel particle as the catalyst and the developed materials showed higher specific capacitance as compared to commercially available CNTs.

Puthusseri et al. ${ }^{209}$ developed Li-based capacitor materials from a waste paper basket using hydrothermal methodology, followed by high temperature pyrolysis. The materials showed a 
Table 4 Types of supercapacitors and the materials used for their electrode fabrication

S. Type of

no. supercapacitor Electrode material

1. Electrochemical Activated carbon nano-materials double layer capacitor (EDLC)

2. Pseudocapacitors Different metal oxides and polymer composite having redox property

3. Hybrid capacitors
For asymmetric hybrid supercapacitors anode Anodic reaction is redox is made up of the same material as in case of reaction while cathode follows pseudo-capacitance ultracapacitors, while cathode is usually made up of activated carbon nano materials

For symmetric hybrid ultracapacitors the metal oxide with activated CNMs or polymer matrix with activated carbon nano-composites
Charge storage mechanism

Non-faradaic charge storage

Redox electrochemical reactions with faradaic current electrochemical double layer mechanism

Redox reactions and electrochemical double layer mechanism is followed.
Merits and demerits

Low specific capacitance \& energy density, fare cyclic stability

Low-rate capability and high-power density, high energy density along with excellent specific capacitance and good cyclic stability

Show high energy density and high-power density with relatively good cyclic stability

High energy density with moderate cyclic stability. very high surface area $\left(2341 \mathrm{~m}^{2} \mathrm{~g}^{-1}\right)$ and hierarchical pore size distributions, where the micro and mesopores are distributed ideally to be function as high-performance supercapacitors. Ion liquid gel polymer was used as the electrolyte for the performance testing of the fabricated device, which showed a power density of $19000 \mathrm{~W} \mathrm{~kg}^{-1}$ and an energy density of $31 \mathrm{~W} \mathrm{~h} \mathrm{~kg}^{-1}$ along with a good energy storage of $61 \mathrm{~W} \mathrm{~h} \mathrm{~kg}^{-1}$. ${ }^{209}$

Dongale et al. demonstrated the modeling of cyclic voltammetry using Artificial Neural Network (ANN), as shown in Fig. 16a. The Levenberg-Marquart propagation algorithm alongside with sigmoid function was used to optimize the power of ANNs. The model established good behavior with an error rate up to $1.24 \%, 1.03 \%, 0.87 \%, 1.17 \%$, and $1.28 \%$ for differently deposited $\mathrm{MnO}_{2} \cdot{ }^{210}$ Wiegert et al. thoroughly evaluated the charging process of the supercapacitor and showed excellent performance with a correlation coefficient of 0.95 (Fig. 16b). ${ }^{211}$ Zhu et al. observed 10000 evaluations for the electrochemical capacitance of the carbonic supercapacitors from almost 1000 articles. They identified five variables, which affects the capacitance of the supercapacitors such as the surface area, pore size, $I_{\mathrm{D}} / I_{\mathrm{G}}$ ratio, doping, and voltage window. ${ }^{212}$

Ma's et al. also predicted the ANN model for the energy storage of electrode devices (Fig. 16c and d) based on the sets of 200 biomass derived carbon nanomaterials and their electrochemical properties. They showed that the specific capacitance can be simulated by features such as aspect ratio, cellulose content, surface area, pore size and volume, internal resistance, and conductance. Random algorithm was used for the calculation of the relative influence on the structural features and the specific capacitance. Machine-based learning predicted the relationship between the morphological features and the performance of available databases. They concluded that the surface area, resistance, pore size, and volume are the utmost significant variables, having a weight of $30.1 \%, 19.4 \%$, and $18.7 \%$, respectively, which affect the electrochemical performance of supercapacitors. ${ }^{144}$

Bridged 3D graphene blocks were used as supercapacitor electrode materials by Zhi et al. ${ }^{205}$ where they reported the existence of Coulombic interaction between CNTs and graphene oxide sheets the as results of $\mathrm{KOH}$ activation. Wong et al. reported the intercalation of CNTs into graphene, which provided a huge surface area and as the result, the obtained material gained high electrical conductivity $\left(39400 \mathrm{~S} \mathrm{~m}^{-1}\right)$ at the mass density of $1.06 \mathrm{~g} \mathrm{~cm}^{-3}$. The energy density of $117.2 \mathrm{~W} \mathrm{~h} \mathrm{~L}^{-1}$ or $110.6 \mathrm{~W} \mathrm{~h} \mathrm{~kg}^{-1}$ and power density of $400 \mathrm{~kW} \mathrm{~kg}^{-1}$ was reported based on the mass density of the active material. ${ }^{213}$

Table 5 depicts the various agricultural waste derived CNMs and their activities in supercapacitor applications. The data shows specific capacitance, current density, and surface area, and strongly depends on the externally activating agents. The reported data indicates the presence of huge surface area with a microporous and meso porous structure, 3D-GO, and 3D-RGO, 2D nanomaterials, hierarchical pore size distributions, variations in mass loadings, flexibility of device along with other structural outlining of morphological variations works as the stimulating agent for enhancement of capacitive properties. On the other hand, the activation achieved by some alkali metal ions works as an inclusive agent for the electrochemical performance of ultracapacitor electrodes. The variation in the structure and states, e.g., hydrogels and flexible arrangements, of electrodes have a huge effect on the outcomes of the electrochemical performances of supercapacitors.

\section{Electrochemical performance testing}

Typically, the electrochemical performance of supercapacitors is evaluated by using either the three-electrode or the twoelectrode system. The three-electrode system is based only on the testing of the minimum amount of active materials by the screening electrode. On the other hand, the two-electrode system is a kind of prototype of the original supercapacitor, where performance is evaluated under ideal conditions.

\subsection{Three-electrode system}

This system is generally used for the study of the electrochemical behavior of active materials. The system consists of a working electrode, a reference electrode, and a counter 


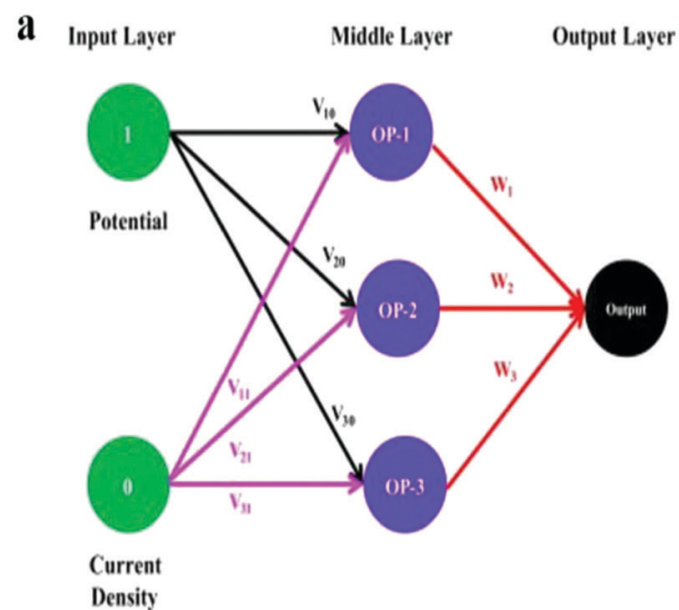

c

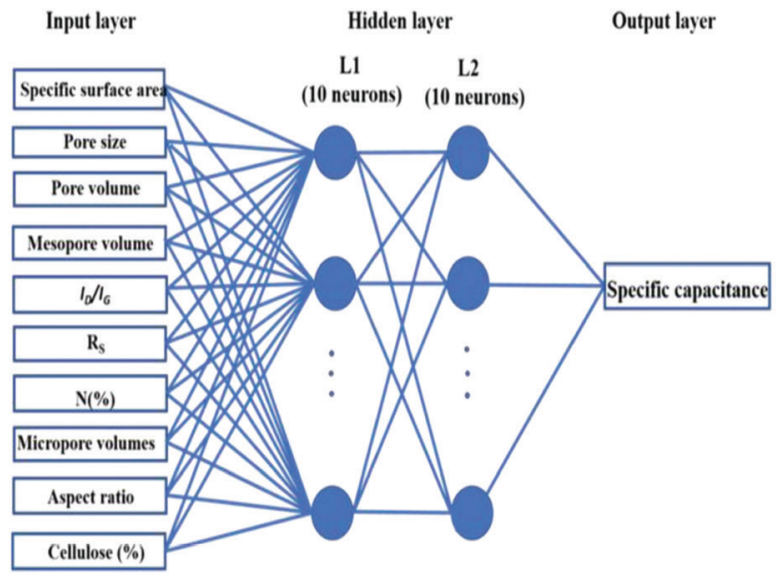

b

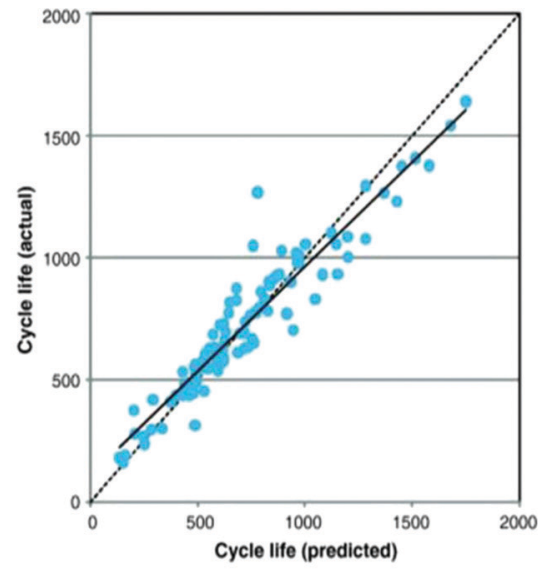

d

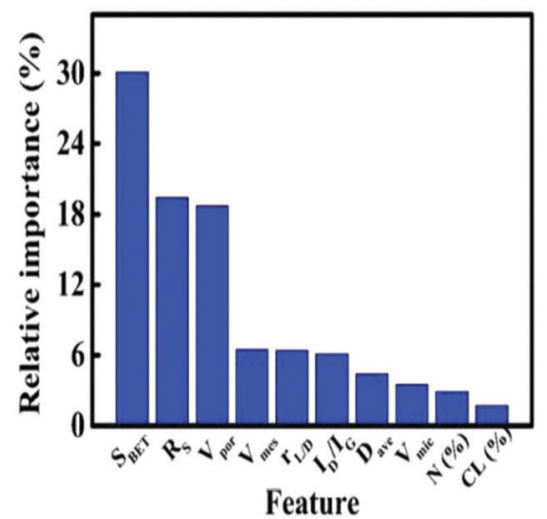

Fig. 16 (a) Multilayer supercapacitor model, ${ }^{132}$ reproduced by permission of Elsevier. (b) Expected cycle life at a discharge current of $2.0 \mathrm{~A},{ }^{132,211}$ reproduced by permission of Elsevier. (c) Artificial model for supercapacitor, (d) importance of inputs and output. ${ }^{132,144}$ Reproduced by permission of Elsevier and RSC.

Table 5 Agriculture based solid waste derived CNMs and their electrochemical performance

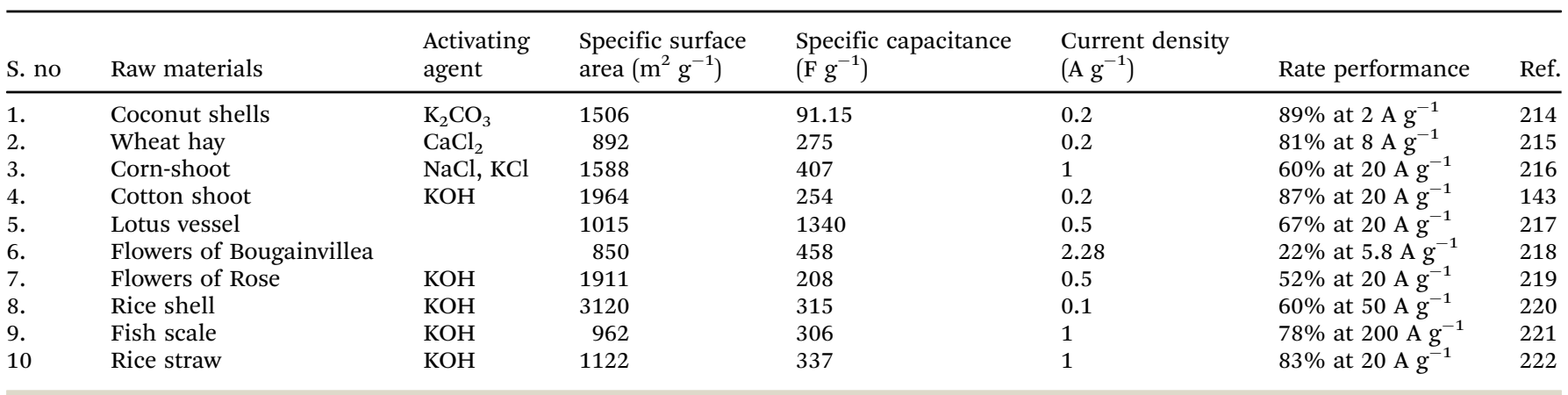

electrode, which are connected with a potentiostat (Fig. 17). The potentiostat is used for controlling the potential of electrodes; it records the change in the current of the electrode concerning the potential. It also controls the current that is passing through an electrode and then records the potential change concerning the current. ${ }^{23,224}$

The working electrode is prepared by the coating of a sticky paste or ink of active materials over the glassy carbon or platinum metal. First, the active material is uniformly dispersed in the appropriate solvent and then coated over the pre-polished working electrode via drop-casting. Further, a small amount of Nafion is added over deposited ink deposition, which prevents the diffusion of ink into the electrolyte. On the other hand, a reference electrode sets up the base potential in the three-electrode system by attaining an unchanged potential. There are different reference electrodes that are used in three-electrode systems such as the 


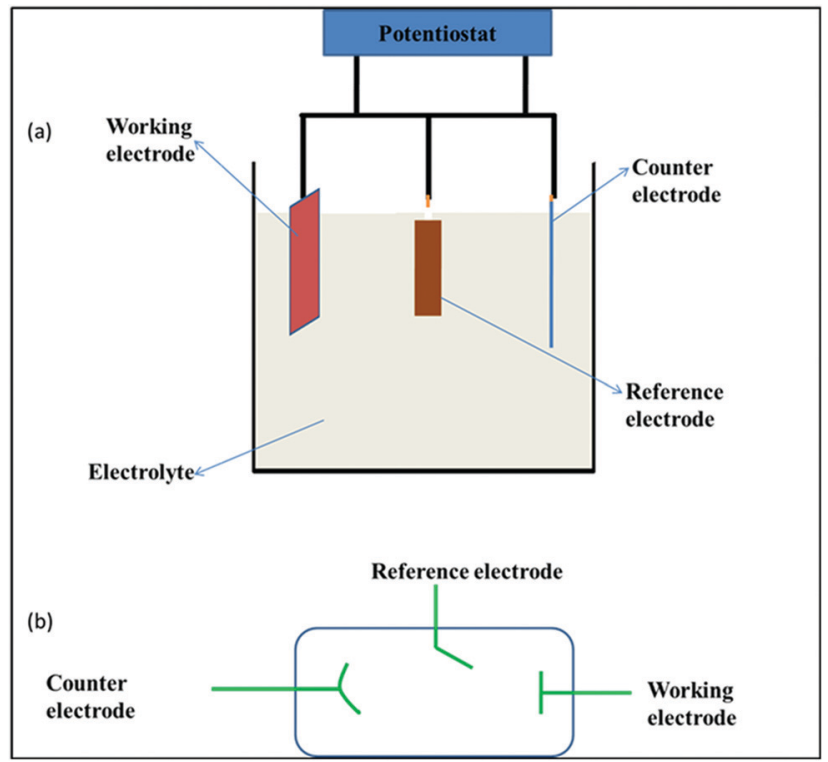

Fig. 17 Schematics presenting the cell connections of the threeelectrode system.

silver chloride electrode $(\mathrm{Ag} / \mathrm{AgCl})$, saturated calomel electrode (SCE), and normal hydrogen electrode (NHE). The final part includes a counter electrode that establishes the balance in the reactions happening at the working electrode by tuning its potential. The CE is generally made of some inert material such as platinum meshes and graphite rods.

\subsection{Two electrode system}

This system is the test fixture configurations for material testing, which can mimic the unit cell and is able to match the performance of packaged supercapacitor cells, thus giving exact characteristics of the active materials. ${ }^{225}$ In the two electrode-system, the two active materials coated electrodes are used, which acted as the cathode and anode. These electrodes are electronically separated from each other by the separators, which avoid short circuit. Also, metal plates, graphite sheets, carbon cloth, and carbon fibers are used as current collectors, which act as a substrate for active materials and are used to establish a connection with a potentiostat. The cathode and anode are generally made by a coating of sticky paste/ink of the active materials (carbon nanomaterials, conducting polymers, and metals oxide) over the current collectors. The active materials may be the same or different for both the electrodes (Fig. 18).

\subsection{Techniques for the evaluation of performance of the supercapacitor}

The performance of supercapacitors is generally measured in terms of specific capacitance $(C)$ in Farads $(F)$, which is directly related to the amount of energy stored. This can be evaluated by cyclic voltammetry, galvanostatic charging/discharging, electrochemical impedance, and spectroscopic techniques. Apart from the specific capacitance, power, and energy density along with

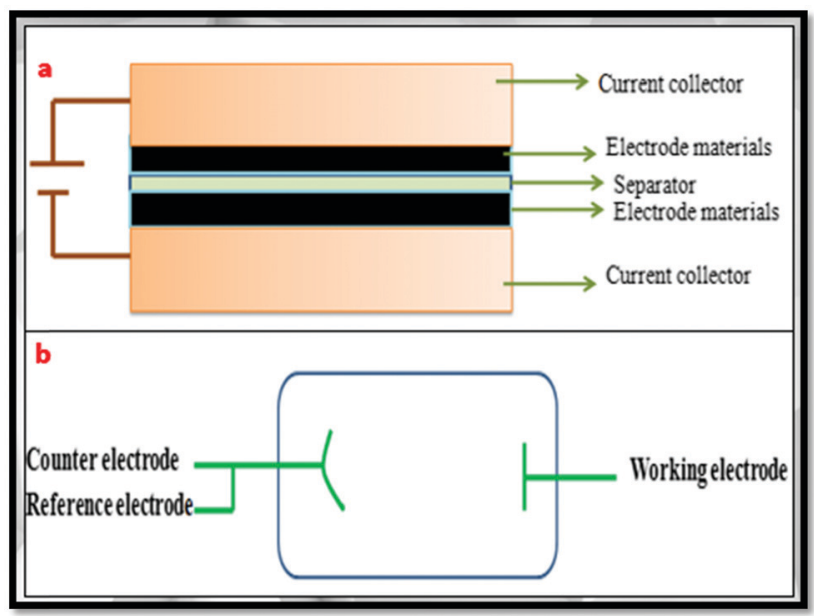

Fig. 18 Schematics presenting the cell connections of the two-electrode system.

cyclic stabilities are also an important parameter for performance testing of the supercapacitor.

7.3.1. Cyclic voltammetry (CV). CV is an important technique that evaluates the plot between the current and potential for calculating the specific capacitance of the supercapacitor and gives the information about EDLC and the pseudocapacitance behavior. Generally, the CV curve shows a perfectly rectangular shape for the ideal capacitor but due to some internal resistance, the CV curve gets deviated from ideality and shows a nearly rectangular shape curve which is thus responsible for EDLC behavior.

The specific capacitance is measured from the current at the center point of potential range $(I)$ where the $\mathrm{CV}$ curve is divided into the two equal halves and scan rate $(v)$ by using the following equation.

$$
C=\frac{I}{m v}
$$

where $C, I, m$, and $v$ represent the specific capacitance, potential range, mass of active materials, and scan rate, respectively. The equation is used for carbon materials and for those materials that show EDLC type behavior.

Conversely, specific capacitance can be evaluated from different equations for pseudocapacitors since pseudocapacitance arises through redox or faradaic charge transfer reaction. Thus, the CV curve deviated from the rectangular shape and shows oxidation and reduction peaks. Hence, the average specific capacitance is measured using voltammetric charge integrated from the $\mathrm{CV}$ curve, according to the following equation.

$$
C=\frac{Q}{2 m V}=\frac{1}{2 m V v} \int_{-}^{+} I(V) \mathrm{d} V
$$

where, $Q$ is the total charge obtained from the area under the curve via the integration of the $\mathrm{CV}$ curve, $\mathrm{m}$ is the mass of active materials coated in electrodes, $v$ is scan rate, $\left(V=V_{+}-V_{-}\right)$ 
and represents the potential window between the positive and negative electrodes. ${ }^{226}$

7.3.2. Electrochemical impedance spectroscopy (EIS). The EIS is a significant technique for evaluating the specific capacitance and equivalent series resistance (ESR) of the supercapacitor. $^{227}$ The EIS is measured at the open-circuit voltage by applying the small amplitude alternative interrupting potential ( $5 \mathrm{mV}$ to $10 \mathrm{mV}$ ) over the frequency range of $10 \mathrm{mHz}$ to $10 \mathrm{kHz}{ }^{227}$ The EIS also gives the relationship between the $f$ and imaginary part of impedance $\left(Z^{\prime}\right)$. The specific capacitance is measured by EIS through the following equation.

$$
C=-\frac{1}{2 \pi f Z^{\prime \prime} m}
$$

where, $f(\mathrm{in} \mathrm{Hz}$ ) and $m$ are the frequency and mass, respectively, of the active materials coated on the electrode.

According to the theoretical concept, in the Nyquist plot, the ideal capacitor exhibits a parallel line to the imaginary axis. In reality, the plot exhibits a line with an inclined angle between 450 and 900 to the real axis, which arose due to the ion diffusion mechanism between Warburg diffusion and pseudocapacitance. ${ }^{228,229}$ This kind of shift from ideal to real by parallel line arises due to the different diffusion depth in the pores and faradaic reaction at the electrodes, which generate abnormal capacitance and pseudocapacitance. ${ }^{230,231}$

7.3.3. Galvanostatic charge-discharge (GCD). The EDLC materials showed a triangular shape curve; on the other hand, pseudocapacitive materials show a non-linear curve due to faradaic reaction. Therefore, the capacitance is calculated for both in different ways. For EDLC, the slope of the discharging part is utilized for calculating the capacitance by using the following equation. $^{225}$

$$
C=\frac{I}{\mathrm{~d} V / \mathrm{d} t}
$$

where, $C, I$, and $\mathrm{d} V / \mathrm{d} t$ are the capacitance, applied current, and slope of the discharging curve, respectively.

On the other hand, the non-linear curve suggested the pseudocapacitance, which arose due to faradaic reaction and the capacitance can be calculated for the following equation without the use of the slope. ${ }^{232}$

$$
C=\frac{(\Delta t)(I)}{\Delta V}
$$

where $\Delta t, I$, and $\Delta V$ are the total discharge time, applied current, and potential difference at the discharging phase, respectively.

7.3.4. Energy density and power density. The energy density (ED) gives information about how much energy store in a given mass or volume and how long the device can perform. The ED of a supercapacitor can be evaluated by the following equation and is expressed in watt-hour per kilogram.

$$
E_{\mathrm{D}}=\frac{1}{2} C(\Delta V)^{2}
$$

where $C$ is the specific capacitance evaluated from the CV or GCD and $\Delta V$ is the operating voltage.
Alternatively, the power density (PD) determines the quickest possibility to deliver the power by the supercapacitor under a constant current. This is expressed in watt per kilogram. The PD of a supercapacitor is evaluated from the following expression.

$$
P_{\mathrm{D}}=\frac{(\Delta V)^{2}}{4 m R_{\mathrm{ESR}}}
$$

where, $\Delta V, m$, and $R_{\mathrm{ESR}}$ are the window potential range, mass of active materials, and $R_{\mathrm{ESR}}$ is the equivalent series resistance (ESR) within the supercapacitor.

7.3.5. Cyclic stability. The stability of a supercapacitor is an important aspect in terms of the electrochemical stability and its applicability in real-time applications. From the commercial point of view, the supercapacitors can produce long life cycles and retain their capacity even after half a million cycles. In general, the cyclic stability test for the supercapacitor performed for 1000 to 10000 cycles, in which one cycle corresponds to the one charge-discharge cycle at a constant current density for the cyclic stability generally performed under the GCD technique at a constant current density. The cyclic stability is important for supercapacitors because a long cyclic run gradually damages the electrode along with the corrosion of its components, which reduced the capacitance and increases the ESR. Therefore, reducing the overall electrochemical performance of the supercapacitor. This helps in the identification of materials that can perform or not in real application fields. Also, the EDLCs-based materials such as activated carbon and carbon nanomaterials show excellent cyclic performance in comparison to the pseudocapacitive materials.

\subsection{Charge storage mechanism of supercapacitors}

The supercapacitor has two type of charge storage mechanisms, which depends upon the way of energy stored, i.e., electrical double-layer capacitors (EDLCs), where the capacitance arises by pure electrostatic charge gathering at the interface of electrode and electrolyte and pseudocapacitors, where the capacitance arises due to the fast and reversible redox reaction in active electrode materials.

7.4.1. Charge storage mechanism of electrical double layer capacitor. Generally, EDLCs have three components, two electrodes, an electrolyte, and a separator. EDLCs shows a conventional capacitor having a charge storage mechanism via electrostatic desirability between the electrolyte ions and charge present at the electrode surface, which permits the formation of an electrical double layer at the interface of the electrode and the electrolyte (Fig. 19). When the EDLCs charge via external load, the cations in the electrolyte move towards the negative electrodes and anions in the electrolyte move towards the positive electrode, whereas the electrons move via the external circuit from the negative to the positive electrode, and the double layer is formed at the interface with the electrode. ${ }^{233,234}$ When the charging is finished, the positive and negative electrodes attract the anions and cations, respectively, from electrolytes towards each other and stabilize the double layer on the electrode surface. ${ }^{235}$ 


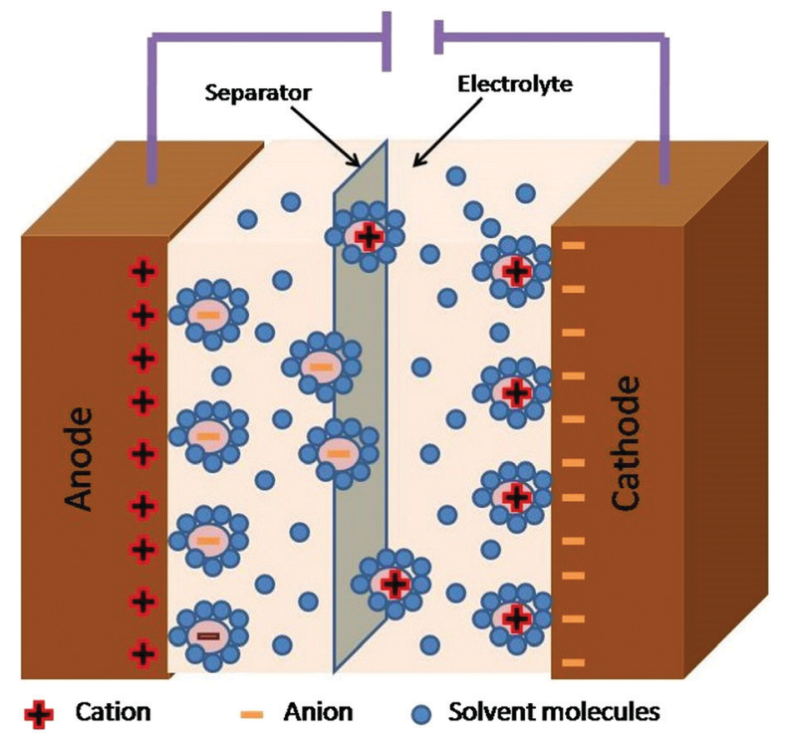

Fig. 19 Schematic representation of EDLCS.

Conversely, the discharging process is just the reverse of the charging process. Such a mechanism of electrical charge generation at the interface of the electrode and electrolyte suggested that the electrolyte concentration is always constant despite the charging and discharging process. Therefore, energy is accumulated at the interface of the electrode and electrolyte.

The amount of energy stored in EDLCs is calculated in the same way as that used in the conventional capacitor by using the following equation.

$$
Q=C V=\frac{A \varepsilon}{d} V
$$

Concerning the conventional capacitor, the capacitance $(C)$ is directly related to the area of plate $(A)$, the permeability of the dielectric $(\varepsilon)$, and is conversely related to the separation $(d)$ between the plates. In general, EDLCs based supercapacitors are made to have a high surface area electrode and use highpermittivity dielectric nature while keeping the current collectors extremely close. Therefore, supercapacitors can attain high specific capacitance in comparison to the conventional capacitor. Due to this, carbon materials such as porous carbon, graphene, and CNTs attain much attention because of their high surface area than metallic strips, which allows storing a higher amount of energy at the collector surface. Besides, the electrodes are electronically separated by the porous separator, which is immersed in the electrolyte medium, and due to higher permeability and close propinquity of the electrodes, the supercapacitors have a low operation voltage of typically $2.5 \mathrm{~V}^{235}$ Also, the shorter distance between the electrodes, which is appreciably higher at the interface of the electrode and electrolyte, enhances the specific capacitance of the EDLCs based supercapacitor. In EDLCs, higher conductively electrolytes are used, which reduce the internal resistance and also increase the wettability of the electrode materials; the increase in the mobility of the electrolyte ions inside the pores of the electrodes further enhance the performance of the EDLC supercapacitor. ${ }^{225,236}$ Usually, various carbon materials are used for EDLCs based supercapacitors but nowadays, carbon nanomaterials such as graphene, CNTs, and other carbon nanostructures are used as electrode materials due to their higher surface area and unique pore size distribution. ${ }^{237,238}$

7.4.2. Charge storage mechanism of pseudocapacitors. These supercapacitors have fast and reversible faradaic charge transfer reactions at the interface between the electrode and electrolyte for energy storage mechanism (Fig. 20); thus, they are called as pseudocapacitors. The occurrence of reversible reaction at the surface of the electrode and the presence of higher surface area along with good electrical conductivity of the active materials are some necessary tools to get high performance in pseudocapacitors. Generally, pseudocapacitance mainly arises from metal oxides such as ruthenium oxides, ${ }^{239}$ vanadium nitride, ${ }^{240}$ manganese oxide, ${ }^{241}$ and conducting polymers such as polyaniline (PANI). ${ }^{242}$ However, carbon nanomaterials such as graphene, CNTs, mesoporous carbon, carbon-based hetero-atoms, ${ }^{243-247}$ and their composite with metal oxides and conductive polymers have shown excellent pseudocapacitance with increased specific capacitance for the fabricated devices. The pseudocapacitor shows 10-100 times higher specific capacitance than the EDLC but it suffers from lower cyclic stability and power density. ${ }^{248}$

The carbon nanomaterials such as graphene and CNTs are used in the pseudocapacitor either in the functionalized form or in the composite form with conducting polymer and metal oxides. The developed composite showed higher performance as compared to their native devices. In particular, the metal oxide-based electrode materials show higher specific capacitance and energy density but these are not economically viable for commercial production and also have lower stability and complexity in porosity tailoring along with low conductivity,

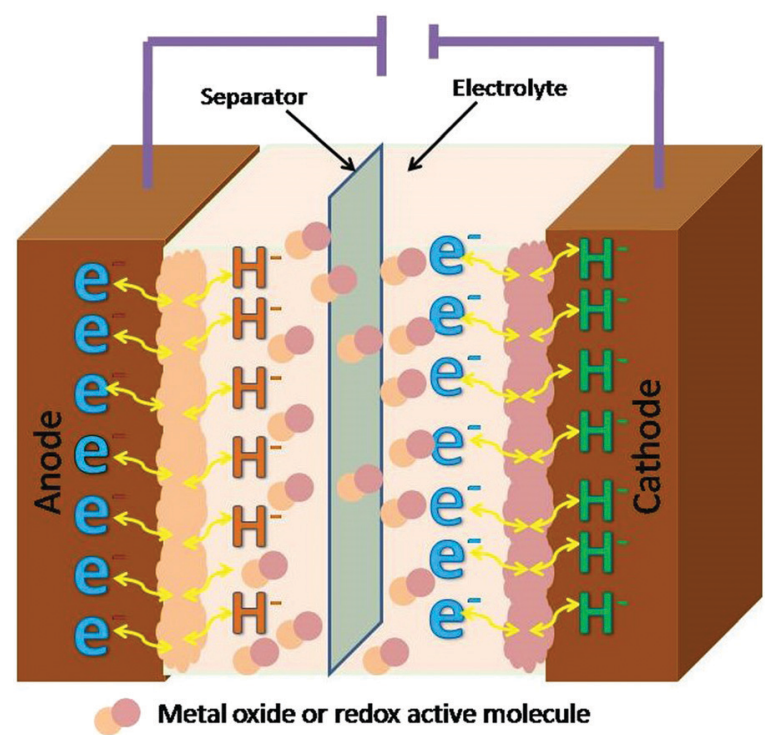

Fig. 20 Schematic representations of pseudocapacitors. 
which has a certain effect on the power density and rate capability performance of the pseudocapacitors. ${ }^{249}$ Conversely, the conducting polymer shows good pseudocapacitive behavior due to their faster charging/discharging rate, appropriate structures, lower cost, and faster doping and undoping process. However, the slow diffusion rate of the electrolytes ions in the polymer network and the volume expansion of the polymers at the time of charging and discharging, reduce their electrochemical performance. ${ }^{250}$ To overcome these glitches, the composites of metal oxides with conductive polymers and carbon nanomaterials have been developed. Such composites can achieve high energy density without compromising their power density because of the involvement of both EDLC (carbon nanomaterials materials) as well as pseudocapacitance (metal oxide and conducting polymers). Besides, these composites-based pseudocapacitors also showed longer life cycles.

\section{Conclusion and future aspects}

The universal status of various solid wastes has come to the time where we can only control waste by its proper utilizations. On the other hand, the foundation of a connection between material science and human world is one of the greatest achievements of nanotechnology, which that can not only deal with such a big environmental issue but also it can make a balance between science and human world. Due to the presence of exceptional carbon contents in various solid waste materials, they can be converted into CNMs, which have distinct incorporative qualities to be applied in various fields, allowing the CNMs and their composite to exhibit their transitional properties.

During the last three decades, various efforts have been made by scientists and researchers to balance the relationship between the waste material based CNMs and various materialistic applications of nanoscience and nanotechnology. The balancing of such a huge issue not only possesses the characteristic that can boost various industries but it can easily provide a myriad of new opportunities for every field of science and technology, which can play a crucial role in the development of human society for decades.

The utilization of waste materials into various CNMs and their further use into polymer nanocomposites, energy storage, energy conservation, drug delivery, and aerospace has increased the waste-based CNMs importance for mankind. Considering the importance of waste materials as promising candidates for the production of CNMs, this review article is an attempt to archive the very recent developments in the field. It includes the utilization of various kinds of waste materials to produce different CNMs with controllable shape, morphologies, physical and chemical properties along with their application potential, methodological changes and others. The structural necessities and other aspects are thoroughly discussed along with their opportunity to regulate the electrochemical to organo-mechanical properties. This review gives an idea about the comparative studies of different waste based CNMs. The review not only clarifies the current universal waste status but also extends the strategies for the production of CNMs with a clearer image of their importance in human life has been also explained.

The waste based CNMs used in different supercapacitors have been justified starting from preliminary to higher studies. While the structural analogy and various methodology have been explained for the justifications of best suitable materials for specific applications. The variations in pore size, pore volume and structure, carbonization, pyrolysis temperature, catalyst compositions and its effects along with the effects of surface morphological changes have been discussed thoroughly. Beside these important aspects, there are some associated drawbacks with these waste based CNMs. For example, the presence of various metals can act as a lethal inclusion for applicative point of view and the toxicity associated with them also play a critical role in many applications. This review highlights different scientific statics that are required to accomplish high specific capacitance and it also includes diverse technical specifications, along with various parameters required to remark on the relative potential of the fabricated device.

The modest synthesis methods, with ecofriendly benefits and cost-effective methodology, are appropriate for the mass scale production of CNMs from solid waste materials. Furthermore, courtesy should be made on the synthesis of different CNMs based composites. The electrochemical performance of 3D porous CNMs and their composites with transition metals and metal oxides might be projected as better alternates for energy storage materials as they have more mobile and sufficient electronic transition. However, to date, there are various studies on the synthesis of CNMs and their doping with heteroatoms, metal ions, metal oxides, and polymers; however, the relation between the mechanical bonding and physical modification of CNMs needs to be examined in depth with the help of computational studies. This will not only be helpful in visualizing the doping patterns but also induce improvement in mechanics and in determining the novel design for the synthesis and manufacture of 3D CNMs. Further, the combination of 3D CNMs with photoluminous nanomaterials, viz., metallic quantum dots and G-dots will explore the potential into some other fields such as bioimaging, energy conservation, and water purification.

The dispersive properties of CNMs play a crucial role in elevating some of the properties such as electrochemical, elasticity and mechanical properties, etc., whereas the mass production of single layer graphene sheets along with good thermal stability is a huge challenge in front of the scientists. The economic production of CNMs is an essential factor for real life applications. Much advancement is needed for the bulk scale production of high quality porous CNMs, which is to be applied in various fields. The absorption of these CNMs into bulk scale industries can become a very impulsive step for the growth of universal economy. However, before processing at the industrial scale, very concise and extensive study should be made on the labor and maintenance cost, resource type, their availability, reproducibility, and associated disadvantages.

\section{Conflicts of interest}

There are no conflicts to declare. 


\section{Acknowledgements}

This work was supported by National Mission on Himalayan Studies (NMHS), GBPNIHE, Almora, India (GBPNI/NMHS-201920/MG), and Department of Science and Technology (DST-FIST), Delhi, India.

\section{References}

1 M. Ripa, G. Fiorentino, H. Giani, A. Clausen and S. Ulgiati, Appl. Energy, 2017, 186, 211-225.

2 H. Zhou, A. Meng, Y. Long, Q. Li and Y. Zhang, Renewable Sustainable Energy Rev., 2014, 36, 107-122.

3 C. Zhuo and Y. A. Levendis, J. Appl. Polym. Sci., 2014, 131, 1-14.

4 G. Berndes, Global Environ. Change., 2002, 12, 253-271.

5 P. Singh Nee Nigam and A. Pandey, Biotechnol. AgroIndustrial Residues Util. Util. Agro-Residues, 2009, pp. 1-466.

6 J. Cook and J. Beyea, Biomass Bioenergy, 2000, 18, 441-455.

7 M. Balat, Energy Sources, Part B, 2007, 2, 167-181.

8 T. Searchinger, R. Heimlich, R. A. Houghton, F. Dong, A. Elobeid, J. Fabiosa, S. Tokgoz, D. Hayes and T. H. Yu, Science, 2008, 319, 1238-1240.

9 N. Moreira, Sci. News, 2005, 168, 218.

10 M. Parikka, Biomass Bioenergy, 2004, 27, 613-620.

11 F. Abnisa and W. M. A. Wan Daud, Energy Convers. Manage., 2014, 87, 71-85.

12 S. Gillet, M. Aguedo, L. Petitjean, A. R. C. Morais, A. M. Da Costa Lopes, R. M. Łukasik and P. T. Anastas, Green Chem., 2017, 19, 4200-4233.

13 G. A. Ferrero, A. B. Fuertes and M. Sevilla, J. Mater. Chem. A, 2015, 3, 2914-2923.

14 Z. Spitalsky, D. Tasis, K. Papagelis and C. Galiotis, Prog. Polym. Sci., 2010, 35, 357-401.

15 J. M. Carlton, J. H. Adams, J. C. Silva, S. L. Bidwell, H. Lorenzi, E. Caler, J. Crabtree, S. V. Angiuoli, E. F. Merino, P. Amedeo, Q. Cheng, R. M. R. Coulson, B. S. Crabb, H. A. Del Portillo, K. Essien, T. V. Feldblyum, C. Fernandez-Becerra, P. R. Gilson, A. H. Gueye, X. Guo, S. Kang'A, T. W. A. Kooij, M. Korsinczky, E. V. S. Meyer, V. Nene, I. Paulsen, O. White, S. A. Ralph, Q. Ren, T. J. Sargeant, S. L. Salzberg, C. J. Stoeckert, S. A. Sullivan, M. M. Yamamoto, S. L. Hoffman, J. R. Wortman, M. J. Gardner, M. R. Galinski, J. W. Barnwell and C. M. Fraser-Liggett, Nature, 2008, 455, 757-763.

16 N. G. Sahoo, S. Rana, J. W. Cho, L. Li and S. H. Chan, Prog. Polym. Sci., 2010, 35, 837-867.

17 X. L. Xie, Y. W. Mai and X. P. Zhou, Mater. Sci. Eng., R, 2005, 49, 89-112.

18 X. Luo, Q. Yang, Y. Dong, X. Huang, D. Kong, B. Wang, H. Liu, Z. Xiao and L. Zhi, J. Mater. Chem. A, 2020, 8, 17558-17567.

19 J. Liang, H. Zhao, L. Yue, G. Fan, T. Li, S. Lu, G. Chen, S. Gao, A. M. Asiri and X. Sun, J. Mater. Chem. A, 2020, 8, 16747-16789.
20 Y. Yang, Y. X. Liu, Y. Li, B. W. Deng, B. Yin and M. B. Yang, J. Mater. Chem. A, 2020, 8, 17257-17265.

21 S. You, X. Xi, X. Zhang, H. Wang, P. Gao, X. Ma, S. Bi, J. Zhang, H. Zhou and Z. Wei, J. Mater. Chem. A, 2020, 8, 17756-17764.

22 C. Liu, Z. Yu, D. Neff, A. Zhamu and B. Z. Jang, Nano Lett., 2010, 10, 4863-4868.

23 A. Izadi-Najafabadi, S. Yasuda, K. Kobashi, T. Yamada, D. N. Futaba, H. Hatori, M. Yumura, S. Iijima and K. Hata, Adv. Mater., 2010, 22, 235-241.

24 K. Liu, M. Wu, H. Jiang, Y. Lin and T. Zhao, J. Mater. Chem. A, 2020, 8, 18802-18809.

25 G. Li, Y. Wang, H. Guo, Z. Liu, P. Chen, X. Zheng, J. Sun, H. Chen, J. Zheng and X. Li, J. Mater. Chem. A, 2020, 8, 16920-16925.

26 J. Zhang and X. S. Zhao, ChemSusChem, 2012, 5, 818-841.

27 F. Paquin, J. Rivnay, A. Salleo, N. Stingelin and C. Silva, J. Mater. Chem. C, 2015, 3, 10715-10722.

28 Q. Cao, H. S. Kim, N. Pimparkar, J. P. Kulkarni, C. Wang, M. Shim, K. Roy, M. A. Alam and J. A. Rogers, Nature, 2008, 454, 495-500.

29 M. Jung, J. Kim, J. Noh, N. Lim, C. Lim, G. Lee, J. Kim, H. Kang, K. Jung, A. D. Leonard, J. M. Tour and G. Cho, IEEE Trans. Electron Devices, 2010, 57, 571-580.

30 X. Li, F. Gittleson, M. Carmo, R. C. Sekol and A. D. Taylor, ACS Nano, 2012, 6, 1347-1356.

31 S. R. Sivakkumar and D.-W. Kim, J. Electrochem. Soc., 2007, 154, A134.

$32 \mathrm{H}$. Wu, K. Wang, Y. Meng, K. Lu and Z. Wei, J. Mater. Chem. A, 2013, 1, 6366-6372.

33 G. Liu, H. Shen, J. Mao, L. Zhang, Z. Jiang, T. Sun, Q. Lan and Z. Zhang, ACS Appl. Mater. Interfaces, 2013, 5, 6909-6914.

34 H. Hu, J. Yu, Y. Li, J. Zhao and H. Dong, J. Biomed. Mater. Res., Part A, 2012, 100A, 141-148.

35 C. Wang, J. Li, C. Amatore, Y. Chen, H. Jiang and X.-M. Wang, Angew. Chem., 2011, 123, 11848-11852.

36 Z. Liu, X. Li, S. M. Tabakman, K. Jiang, S. Fan and H. Dai, J. Am. Chem. Soc., 2008, 130, 13540-13541.

37 D. Pissuwan, S. M. Valenzuela and M. B. Cortie, Trends Biotechnol., 2006, 24, 62-67.

38 G. Pagona and N. Tagmatarchis, Curr. Med. Chem., 2006, 13, 1789-1798.

39 O. Gohardani, M. C. Elola and C. Elizetxea, Prog. Aerosp. Sci., 2014, 70, 42-68.

40 T. Natsuki, T. Tsuchiya, Q. Q. Ni and M. Endo, Carbon, 2010, 48, 4362-4368.

41 A. L. Ramaswamy, P. Kaste, A. W. Miziolek, B. Homan, S. Trevino and M. A. O'Keefe, Am. Chem. Soc., 2005, 180-197.

42 N. M. Barkoula, B. Alcock, N. O. Cabrera and T. Peijs, Polym. Polym. Compos., 2008, 16, 101-113.

43 Y. Zhao, R. Nakamura, K. Kamiya, S. Nakanishi and K. Hashimoto, Nat. Commun., 2013, 4, 1-7.

44 K. Tarawneh and N. Al-Aqtash, J. Comput. Theor. Nanosci., 2013, 10, 2080-2087.

45 D. Yu, E. Nagelli, F. Du and L. Dai, J. Phys. Chem. Lett., 2010, 1, 2165-2173. 
46 K. R. S. Chandrakumar, K. Srinivasu and S. K. Ghosh, J. Phys. Chem. C, 2008, 112, 15670-15679.

47 Z. Yang, H. Nie, X. Chen, X. Chen and S. Huang, J. Power Sources, 2013, 236, 238-249.

48 B. R. Sathe, X. Zou and T. Asefa, Catal. Sci. Technol., 2014, 4, 2023-2030.

49 W. K. Maboya, N. J. Coville and S. D. Mhlanga, S. Afr. J. Chem., 2016, 69, 15-26.

50 A. J. Clancy, M. K. Bayazit, S. A. Hodge, N. T. Skipper, C. A. Howard and M. S. P. Shaffer, Chem. Rev., 2018, 118, 7363-7408.

51 Z. Zhao, M. Li, L. Zhang, L. Dai and Z. Xia, Adv. Mater., 2015, 27, 6834-6840.

52 J. D. Ryan, A. Lund, A. I. Hofmann, R. Kroon, R. SarabiaRiquelme, M. C. Weisenberger and C. Müller, ACS Appl. Energy Mater., 2018, 1, 2934-2941.

53 R. Pang, M. Li and C. Zhang, Talanta, 2015, 131, 38-45.

54 E. Flahaut, L. Evariste, L. Gauthier, C. Larue, C. Line, E. Meunier and F. Mouchet, Techniques de l'Ingénieur, 2018, 1-34.

55 T. Sato, Y. Suda, H. Uruno, H. Takikawa, H. Tanoue, H. Ue, N. Aoyagi, T. Okawa and K. Shimizu, J. Phys.: Conf. Ser., 2012, 352, 012032.

56 R. K. Emmett, M. Karakaya, R. Podila, M. R. Arcila-Velez, J. Zhu, A. M. Rao and M. E. Roberts, J. Phys. Chem. C, 2014, 118, 26498-26503.

57 Z. L. Xu, J. K. Kim and K. Kang, Nano Today, 2018, 19, 84-107.

$58 \mathrm{~W}$. Lu, High performance batteries with carbon nanomaterials and ionic liquids, US Pat. 8236446, ADA Technologies Inc., 2012.

59 H. K. Liu, G. X. Wang, Z. Guo, J. Wang and K. Konstantinov, J. Nanosci. Nanotechnol., 2006, 6, 1-15.

60 T. Chen and L. Dai, Mater. Today, 2013, 16, 272-280.

61 B. K. Kim, V. Chabot and A. Yu, Electrochim. Acta, 2013, 109, 370-380.

62 A. Lund, N. M. van der Velden, N. K. Persson, M. M. Hamedi and C. Müller, Mater. Sci. Eng., R, 2018, 126, 1-29.

63 B. L. Johnson and C. DeRosa, Rev. Environ. Health, 1997, 12, 235-251.

64 A. C. Upton, T. Kneip and P. Toniolo, Annu. Rev. Public Health, 1989, 10, 1-25.

65 A. Biggeri, F. Barbone, C. Lagazio, M. Bovenzi and G. Stanta, Environ. Health Perspect., 1996, 104, 750-754.

66 P. Comba, V. Ascoli, S. Belli, M. Benedetti, L. Gatti, P. Ricci and A. Tieghi, Occup. Environ. Med., 2003, 60, 680-683.

67 P. Zambon, P. Ricci, E. Bovo, A. Casula, M. Gattolin, A. R. Fiore, F. Chiosi and S. Guzzinati, Environ. Health: Global Access Sci. Source, 2007, 6, 1-10.

68 P. Elliott, G. Shaddick, I. Kleinschmidt, D. Jolley, P. Walls, J. Beresford and C. Grundy, Br. J. Cancer, 1996, 73, 702-710.

69 T. R. Hsiue, S. S. Lee and H. I. Chen, Chest, 1991, 100, 698-702.

70 Y. Miyake, A. Yura, H. Misaki, Y. Ikeda, T. Usui, M. Iki and T. Shimizu, Eur. J. Epidemiol., 2005, 20, 1023-1029.
71 C. Zhuo and Y. A. Levendis, J. Appl. Polym. Sci., 2014, 131, 1-14.

72 C. S. Burke, E. Salas, K. Smith-Jentsch and M. A. Rosen, Macrocognition Metrics Scenar. Des. Eval. Real-World Teams, 2012, pp. 29-43.

73 C. S. Burke, E. Salas, K. Smith-Jentsch and M. A. Rosen, Macrocognition Metrics Scenar. Des. Eval. Real-World Teams, 2012, pp. 29-43.

74 I. J. Ahluwalia and U. Patel, Indian Counc. Res. Int. Econ. Relations, 2018, pp. 1-48.

75 D. M. C. Chen, B. L. Bodirsky, T. Krueger, A. Mishra and A. Popp, Environ. Res. Lett., 2020, 15(7), 074021.

76 F. Akbar, M. Kolahdouz, S. Larimian, B. Radfar and H. H. Radamson, J. Mater. Sci.: Mater. Electron., 2015, 26, 4347-4379.

77 J. M. Allen, T. C. Vincent and K. B. Richard, Chem. Rev., 2010, 110, 132-145.

78 E. Pop, V. Varshney and A. K. Roy, MRS Bull., 2012, 37, 1273-1281.

79 T. V. Cuong, V. H. Pham, Q. T. Tran, J. S. Chung, E. W. Shin, J. S. Kim and E. J. Kim, Mater. Lett., 2010, 64, 765-767.

80 N. A. El Essawy, S. M. Ali, H. A. Farag, A. H. Konsowa, M. Elnouby and H. A. Hamad, Ecotoxicol. Environ. Saf., 2017, 145, 57-68.

81 J. Gong, J. Liu, X. Wen, Z. Jiang, X. Chen, E. Mijowska and T. Tang, Ind. Eng. Chem. Res., 2014, 53, 4173-4181.

82 H. Wang, H. Yi, C. Zhu, X. Wang and H. J. Fan, Nano Energy, 2015, 13, 658-669.

83 Z. Bi, Q. Kong, Y. Cao, G. Sun, F. Su, X. Wei, X. Li, A. Ahmad, L. Xie and C. M. Chen, J. Mater. Chem. A, 2019, 7, 16028-16045.

84 A. R. Kamali, J. Yang and Q. Sun, Appl. Surf. Sci., 2019, 476, 539-551.

85 Y. Wen, K. Kierzek, X. Chen, J. Gong, J. Liu, R. Niu, E. Mijowska and T. Tang, Waste Manage., 2019, 87, 691-700.

86 S. Pandey, M. Karakoti, S. Dhali, N. Karki, B. SanthiBhushan, C. Tewari, S. Rana, A. Srivastava, A. B. Melkani and N. G. Sahoo, Waste Manage., 2019, 88, 48-55.

87 C. Tewari, G. Tatrari, M. Karakoti, S. Pandey, M. Pal, S. Rana, B. SanthiBhushan, A. B. Melkani, A. Srivastava and N. G. Sahoo, Mater. Sci. Eng., C, 2019, 104, 109970.

88 X. Y. Chen, L. X. Cheng, X. Deng, L. Zhang and Z. J. Zhang, Ind. Eng. Chem. Res., 2014, 53, 6990-6997.

89 L. Cui, X. Wang, N. Chen, B. Ji and L. Qu, Nanoscale, 2017, 9, 9089-9094.

90 R. Papon, C. Pierlot, S. Sharma, S. M. Shinde, G. Kalita and M. Tanemura, Phys. Status Solidi B, 2017, 254, 1-7.

91 L. Cui, X. Wang, N. Chen, B. Ji and L. Qu, Nanoscale, 2017, 9, 9089-9094.

92 S. Sharma, G. Kalita, R. Hirano, S. M. Shinde, R. Papon, H. Ohtani and M. Tanemura, Carbon, 2014, 72, 66-73.

93 Z. Baig, O. Mamat and M. Mustapha, Crit. Rev. Solid State Mater. Sci., 2018, 43, 1-46.

94 V. N. Popov, Mater. Sci. Eng., R, 2004, 43, 61-102. 
95 N. Zhang, Y. Zhang, M. Q. Yang, Z. R. Tang and Y. J. Xu, J. Catal., 2013, 299, 210-221.

96 O. G. Apul, Q. Wang, Y. Zhou and T. Karanfil, Water Res., 2013, 47, 1648-1654.

97 J. Appenzeller, J. Knoch, R. Martel, V. Derycke, S. J. Wind and P. Avouris, IEEE Trans. Nanotechnol., 2002, 1, 184-188.

98 G. Bajad, V. Guguloth, R. P. Vijayakumar and S. Bose, Fullerenes, Nanotubes, Carbon Nanostruct., 2016, 24, 162-169.

99 N. Mishra, G. Das, A. Ansaldo, A. Genovese, M. Malerba, M. Povia, D. Ricci, E. Di Fabrizio, E. Di Zitti, M. Sharon and M. Sharon, J. Anal. Appl. Pyrolysis, 2012, 94, 91-98.

100 V. G. Pol and P. Thiyagarajan, J. Environ. Monit., 2010, 12, 455-459.

101 C. Wu, Z. Wang, L. Wang, P. T. Williams and J. Huang, RSC Adv., 2012, 2, 4045-4047.

102 Z. Jiang, R. Song, W. Bi, J. Lu and T. Tang, Carbon, 2007, 45, 449-458.

103 J. Gong, J. Liu, L. Ma, X. Wen, X. Chen, D. Wan, H. Yu, Z. Jiang, E. Borowiak-Palen and T. Tang, Appl. Catal., B, 2012, 117-118, 185-193.

104 K. Mukhopadhyay, A. Koshio, N. Tanaka and H. Shinohara, Jpn. J. Appl. Phys., 1998, 37, L1257.

105 C. Zhuo, J. O. Alves, J. A. S. Tenorio and Y. A. Levendis, Ind. Eng. Chem. Res., 2012, 51, 2922-2930.

106 J. Liu, Z. Jiang, H. Yu and T. Tang, Polym. Degrad. Stab., 2011, 96, 1711-1719.

107 M. Hassan, E. Haque, K. R. Reddy, A. I. Minett, J. Chen and V. G. Gomes, Nanoscale, 2014, 6, 11988-11994.

108 H. Sun, N. Gao, K. Dong, J. Ren and X. Qu, ACS Nano, 2014, 8, 6202-6210.

109 L. Li, G. Wu, G. Yang, J. Peng, J. Zhao and J. J. Zhu, Nanoscale, 2013, 5, 4015-4039.

110 S. Kim, S. W. Hwang, M. K. Kim, D. Y. Shin, D. H. Shin, C. O. Kim, S. B. Yang, J. H. Park, E. Hwang, S. H. Choi, G. Ko, S. Sim, C. Sone, H. J. Choi, S. Bae and B. H. Hong, ACS Nano, 2012, 6, 8203-8208.

111 J. Peng, W. Gao, B. K. Gupta, Z. Liu, R. Romero-Aburto, L. Ge, L. Song, L. B. Alemany, X. Zhan, G. Gao, S. A. Vithayathil, B. A. Kaipparettu, A. A. Marti, T. Hayashi, J. J. Zhu and P. M. Ajayan, Nano Lett., 2012, 12, 844-849.

112 V. Gupta, N. Chaudhary, R. Srivastava, G. D. Sharma, R. Bhardwaj and S. Chand, J. Am. Chem. Soc., 2011, 133, 9960-9963.

113 M. Kaur, M. Kaur and V. K. Sharma, Adv. Colloid Interface Sci., 2018, 259, 44-64.

114 Y. Du, P. Xiao, J. Yuan and J. Chen, Coatings, 2020, 109, 892.

115 J. Wei, X. Zhang, Y. Sheng, J. Shen, P. Huang, S. Guo, J. Pan, B. Liu and B. Feng, New J. Chem., 2014, 38, 906-909.

116 Y. Hu, J. Yang, J. Tian, L. Jia and J. S. Yu, RSC Adv., 2014, 4, 47169-47176.

117 A. Kumari, A. Kumar, S. K. Sahu and S. Kumar, Sens. Actuators, B, 2018, 254, 197-205.

118 S. Y. Park, H. U. Lee, E. S. Park, S. C. Lee, J. W. Lee, S. W. Jeong, C. H. Kim, Y. C. Lee, Y. S. Huh and J. Lee, ACS Appl. Mater. Interfaces, 2014, 6, 3365-3370.
119 S. Thambiraj and D. R. Shankaran, Appl. Surf. Sci., 2016, 390, 435-443.

120 A. Prasannan and T. Imae, Ind. Eng. Chem. Res., 2013, 52, 15673-15678.

121 A. Tyagi, K. M. Tripathi, N. Singh, S. Choudhary and R. K. Gupta, RSC Adv., 2016, 6, 72423-72432.

122 P. Jegannathan, A. TermehYousefi, M. S. A. Karim and N. A. Kadri, Probl. Biocybern. Biomed. Eng., 2018, 38, 481-497.

123 L. Sun, C. Tian, M. Li, X. Meng, L. Wang, R. Wang, J. Yin and H. Fu, J. Mater. Chem. A, 2013, 1, 6462-6470.

124 X. Zhang, K. Zhang, H. Li, Q. Cao, L. Jin and P. Li, J. Power Sources, 2017, 344, 176-184.

125 C. K. Liu, Y. Feng, H. J. He, J. Zhang, R. J. Sun and M. Y. Chen, Mater. Des., 2015, 85, 483-486.

126 F. Paquin, J. Rivnay, A. Salleo, N. Stingelin and C. Silva, J. Mater. Chem. C, 2015, 3, 10715-10722.

127 H. Chen, F. Yu, G. Wang, L. Chen, B. Dai and S. Peng, ACS Omega, 2018, 3, 4724-4732.

128 X. Q. Lin, Q. F. Lü, Q. Li, M. Wu and R. Liu, ACS Omega, 2018, 3, 13283-13289.

129 Y. Zhang, L. Liu, P. Zhang, J. Wang, M. Xu, Q. Deng, Z. Zeng and S. Deng, Chem. Eng. J., 2019, 355, 309-319.

130 J. Xia, N. Zhang, S. Chong, D. Li, Y. Chen and C. Sun, Green Chem., 2018, 20, 694-700.

131 M. Karnan, K. Subramani, N. Sudhan, N. Ilayaraja and M. Sathish, ACS Appl. Mater. Interfaces, 2016, 8, 35191-35202.

132 J. Wang, X. Zhang, Z. Li, Y. Ma and L. Ma, J. Power Sources, 2020, 451, 227794.

133 O. Norouzi, S. Jafarian, F. Safari, A. Tavasoli and B. Nejati, Bioresour. Technol., 2016, 219, 643-651.

134 Y. Huang, Y. Liu, G. Zhao and J. Y. Chen, J. Mater. Sci., 2017, 52, 478-488.

135 C. Wang, D. Wu, H. Wang, Z. Gao, F. Xu and K. Jiang, J. Power Sources, 2017, 363, 375-383.

136 Y. Gong, D. Li, C. Luo, Q. Fu and C. Pan, Green Chem., 2017, 19, 4132-4140.

137 M. Zhang, Y. Li, H. Si, B. Wang and T. Song, Int. J. Electrochem. Sci., 2017, 12, 7844-7852.

138 X. Tian, H. Ma, Z. Li, S. Yan, L. Ma, F. Yu, G. Wang, X. Guo, Y. Ma and C. Wong, J. Power Sources, 2017, 359, 88-96.

139 A. K. Mondal, K. Kretschmer, Y. Zhao, H. Liu, C. Wang, B. Sun and G. Wang, Chem. - Eur. J., 2017, 23, 3683-3690.

140 S. Gao, Y. Chen, H. Fan, X. Wei, C. Hu, H. Luo and L. Qu, J. Mater. Chem. A, 2014, 2, 3317-3324.

141 C. Wang, J. Huang, H. Qi, L. Cao, Z. Xu, Y. Cheng, X. Zhao and J. Li, J. Power Sources, 2017, 358, 85-92.

142 L. Sun, C. Tian, M. Li, X. Meng, L. Wang, R. Wang, J. Yin and H. Fu, J. Mater. Chem. A, 2013, 1, 6462-6470.

143 X. Tian, H. Ma, Z. Li, S. Yan, L. Ma, F. Yu, G. Wang, X. Guo, Y. Ma and C. Wong, J. Power Sources, 2017, 359, 88-96.

144 J. Wang, Z. Li, S. Yan, X. Yu, Y. Ma and L. Ma, RSC Adv., 2019, 9, 14797-14808.

145 J. Cui, Y. Xi, S. Chen, D. Li, X. She, J. Sun, W. Han, D. Yang and S. Guo, Adv. Funct. Mater., 2016, 26, 8487-8495. 
146 R. Thangavel, K. Kaliyappan, H. V. Ramasamy, X. Sun and Y. S. Lee, ChemSusChem, 2017, 10, 2805-2815.

147 C. Wang, D. Wu, H. Wang, Z. Gao, F. Xu and K. Jiang, J. Mater. Chem. A, 2018, 6, 1244-1254.

148 L. Zheng, S. Wang, Y. Yang, X. Fu, T. Jiang and J. Yang, ACS Omega, 2019, 4, 5904-5914.

149 G. Zhu, L. Ma, H. Lv, Y. Hu, T. Chen, R. Chen, J. Liang, X. Wang, Y. Wang, C. Yan, Z. Tie, Z. Jin and J. Liu, Nanoscale, 2017, 9, 1237-1243.

150 D. Yan, C. Yu, D. Li, X. Zhang, J. Li, T. Lu and L. Pan, J. Mater. Chem. A, 2016, 4, 11077-11085.

151 R. R. Gaddam, D. Yang, R. Narayan, K. V. S. N. Raju, N. A. Kumar and X. S. Zhao, Nano Energy, 2016, 26, 346-352.

152 T. E. Rufford, D. Hulicova-Jurcakova, K. Khosla, Z. Zhu and G. Q. Lu, J. Power Sources, 2010, 195, 912-918.

153 L. Tao, Y. Huang, Y. Zheng, X. Yang, C. Liu, M. Di, S. Larpkiattaworn, M. R. Nimlos and Z. Zheng, J. Taiwan Inst. Chem. Eng., 2019, 95, 217-226.

154 B. Liu, X. Zhou, H. Chen, Y. Liu and H. Li, Electrochim. Acta, 2016, 208, 55-63.

155 C. Long, X. Chen, L. Jiang, L. Zhi and Z. Fan, Nano Energy, 2015, 12, 141-151.

156 L. Wang, G. Mu, C. Tian, L. Sun, W. Zhou, P. Yu, J. Yin and H. Fu, ChemSusChem, 2013, 6, 880-889.

157 X. Zheng, W. Lv, Y. Tao, J. Shao, C. Zhang, D. Liu, J. Luo, D. W. Wang and Q. H. Yang, Chem. Mater., 2014, 26, 6896-6903.

158 P. Hao, Z. Zhao, J. Tian, H. Li, Y. Sang, G. Yu, H. Cai, H. Liu, C. P. Wong and A. Umar, Nanoscale, 2014, 6, 12120-12129.

159 Y. Fan, P. Liu, B. Zhu, S. Chen, K. Yao and R. Han, Int. J. Hydrogen Energy, 2015, 40, 6188-6196.

$160 \mathrm{~W}$. Gu and G. Yushin, Wiley Interdiscip. Rev.: Energy Environ., 2014, 3, 424-473.

161 S. He and W. Chen, J. Power Sources, 2015, 294, 150-158.

162 C. Zhang, Z. Geng, M. Cai, J. Zhang, X. Liu, H. Xin and J. Ma, Int. J. Hydrogen Energy, 2013, 38, 9243-9250.

163 S. I. Mussatto, E. M. S. Machado, S. Martins, J. A. Teixeira, J. Fermoso, O. Mašek, F. Codignole Luz, M. Volpe, L. Fiori, A. Manni, S. Cordiner, V. Mulone, V. Rocco, S. Park, S. J. Kim, K. C. Oh, L. Cho, M. J. Kim, I. S. Jeong, C. G. Lee, D. H. Kim, N. Kondamudi, S. K. Mohapatra, M. Misra, D. Pujol, C. Liu, J. Gominho, M. À. Olivella, N. Fiol, I. Villaescusa, H. Pereira, T. M. Mata, A. A. Martins, N. S. Caetano, C. L. Mendoza Martinez, E. P. Alves Rocha, A. de C. Oliveira Carneiro, F. J. Borges Gomes, L. A. RibasBatalha, E. Vakkilainen, M. Cardoso and E. B. Conference, Bioresour. Technol., 2018, 256, 11757-11760.

164 K. Yang, J. Peng, C. Srinivasakannan, L. Zhang, H. Xia and X. Duan, Bioresour. Technol., 2010, 101, 6163-6169.

165 X. He, P. Ling, M. Yu, X. Wang, X. Zhang and M. Zheng, Electrochim. Acta, 2013, 105, 635-641.

166 G. A. M. Ali, S. A. B. A. Manaf, A. Kumar, K. F. Chong and G. Hegde, J. Phys. D: Appl. Phys., 2014, 47, 495307.

167 Y. Fan, X. Yang, B. Zhu, P. F. Liu and H. T. Lu, J. Power Sources, 2014, 268, 584-590.
168 K. L. Hong, L. Qie, R. Zeng, Z. Q. Yi, W. Zhang, D. Wang, W. Yin, C. Wu, Q. J. Fan, W. X. Zhang and Y. H. Huang, J. Mater. Chem. A, 2014, 2, 12733-12738.

169 E. Frackowiak, Phys. Chem. Chem. Phys., 2007, 9, 1774-1785.

170 D. W. Wang, F. Li, M. Liu, G. Q. Lu and H. M. Cheng, J. Phys. Chem. C, 2008, 112, 9950-9955.

171 C. Wang, Y. Xiong, H. Wang, C. Jin and Q. Sun, J. Mater. Chem. A, 2017, 5, 15759-15770.

172 L. L. Zhang, H. H. Li, Y. H. Shi, C. Y. Fan, X. L. Wu, H. F. Wang, H. Z. Sun and J. P. Zhang, ACS Appl. Mater. Interfaces, 2016, 8, 4233-4241.

173 J. Hou, C. Cao, F. Idrees and X. Ma, ACS Nano, 2015, 9, 2556-2564.

174 D. D. Zhou, W. Y. Li, X. L. Dong, Y. G. Wang, C. X. Wang and Y. Y. Xia, J. Mater. Chem. A, 2013, 1, 8488-8496.

175 H. Lu, L. Zhuang, R. R. Gaddam, X. Sun, C. Xiao, T. Duignan, Z. Zhu and X. S. Zhao, J. Mater. Chem. A, 2019, 7, 22579-22587.

176 M. Liu, K. Zhang, M. Si, H. Wang, L. Chai and Y. Shi, Carbon, 2019, 153, 707-716.

177 Z. Ling, Z. Wang, M. Zhang, C. Yu, G. Wang, Y. Dong, S. Liu, Y. Wang and J. Qiu, Adv. Funct. Mater., 2016, 26, 111-119.

178 J. Zhao, Y. Li, G. Wang, T. Wei, Z. Liu, K. Cheng, K. Ye, K. Zhu, D. Cao and Z. Fan, J. Mater. Chem. A, 2017, 5, 23085-23093.

179 C. Huang, T. Sun and D. Hulicova-Jurcakova, ChemSusChem, 2013, 6, 2330-2339.

180 H. Xu, C. Wu, X. Wei and S. Gao, J. Mater. Chem. A, 2018, 6, 15340-15347.

181 E. P. Randviir, D. A. C. Brownson and C. E. Banks, Mater. Today, 2014, 17, 426-432.

182 G. Eda, G. Fanchini and M. Chhowalla, Nat. Nanotechnol., 2008, 3, 270-274.

183 K. S. Kim, Y. Zhao, H. Jang, S. Y. Lee, J. M. Kim, K. S. Kim, J. H. Ahn, P. Kim, J. Y. Choi and B. H. Hong, Nature, 2009, 457, 706-710.

184 S. Bae, H. Kim, Y. Lee, X. Xu, J. S. Park, Y. Zheng, J. Balakrishnan, T. Lei, H. Ri Kim, Y. Il Song, Y. J. Kim, K. S. Kim, B. Özyilmaz, J. H. Ahn, B. H. Hong and S. Iijima, Nat. Nanotechnol., 2010, 5, 574-578.

185 T. Hasan, V. Scardaci, P. H. Tan, F. Bonaccorso, A. G. Rozhin, Z. Sun and A. C. Ferrari, Molecular-and Nano-Tubes, 2011.

186 M. Beidaghi and Y. Gogotsi, Energy Environ. Sci., 2014, 7, 867-884.

187 C. Lee, X. Wei, J. W. Kysar and J. Hone, Science, 2008, 321, 385-388.

188 R. K. Joshi, P. Carbone, F. C. Wang, V. G. Kravets, Y. Su, I. V. Grigorieva, H. A. Wu, A. K. Geim and R. R. Nair, Science, 2014, 343, 752-754.

189 M. A. Garakani, S. Abouali, Z. L. Xu, J. Huang, J. Q. Huang and J. K. Kim, J. Mater. Chem. A, 2017, 5, 3547-3557.

190 W. G. Chong, J. Q. Huang, Z. L. Xu, X. Qin, X. Wang and J. K. Kim, Adv. Funct. Mater., 2016, 27(4), 1604815.

191 Y. Lian, M. Ni, Z. Huang, R. Chen, L. Zhou, W. Utetiwabo and W. Yang, Chem. Eng. J., 2019, 366, 313-320. 
192 Z. Chen, X. Wang, B. Xue, Q. Wei, L. Hu, Z. Wang, X. Yang and J. Qiu, ChemSusChem, 2019, 12, 1390-1400.

193 H. Peng, G. Ma, K. Sun, Z. Zhang, Q. Yang and Z. Lei, Electrochim. Acta, 2016, 190, 862-871.

194 Y. Li, X. Wang and M. Cao, J. CO 2 Util., 2018, 27, 204-216.

195 Z. Zhang, Y. Liu, Z. Huang, L. Ren, X. Qi, X. Wei and J. Zhong, Phys. Chem. Chem. Phys., 2015, 17, 20795-20804.

196 W. Xing, S. Z. Qiao, R. G. Ding, F. Li, G. Q. Lu, Z. F. Yan and H. M. Cheng, Carbon, 2006, 44, 216-224.

197 P. Deng, S. Lei, W. Wang, W. Zhou, X. Ou, L. Chen, Y. Xiao and B. Cheng, J. Mater. Sci., 2018, 53, 14536-14547.

198 R. Jalili, D. Esrafilzadeh, S. H. Aboutalebi, Y. M. Sabri, A. E. Kandjani, S. K. Bhargava, E. D. Gaspera, T. R. Gengenbach, A. Walker, Y. Chao and C. Wang, Nat. Commun., 2018, 9, 1-13.

199 B. Campbell and J. Manning, Rise Vict. Cult. Microaggressions, Safe Spaces, New Cult. Wars, 2018, pp. 1-265.

200 D. Prasai, J. C. Tuberquia, R. R. Harl, G. K. Jennings, B. R. Rogers and K. I. Bolotin, ACS Nano, 2012, 6, 4540.

201 A. S. Mayorov, R. V. Gorbachev, S. V. Morozov, L. Britnell, R. Jalil, L. A. Ponomarenko, P. Blake, K. S. Novoselov, K. Watanabe, T. Taniguchi and A. K. Geim, Nano Lett., 2011, 11, 2396-2399.

202 A. K. Mondal, K. Kretschmer, Y. Zhao, H. Liu, H. Fan and G. Wang, Microporous Mesoporous Mater., 2017, 246, 72-80.

203 C. Zequine, C. K. Ranaweera, Z. Wang, S. Singh, P. Tripathi, O. N. Srivastava, B. K. Gupta, K. Ramasamy, P. K. Kahol, P. R. Dvornic and R. K. Gupta, Sci. Rep., 2016, 6, 1-10.

204 N. Mishra, S. Shinde, R. Vishwakarma, S. Kadam, M. Sharon and M. Sharon, AIP Conf. Proc., 2013, 1538, 228-236.

205 M. Zhi, F. Yang, F. Meng, M. Li, A. Manivannan and N. Wu, ACS Sustainable Chem. Eng., 2014, 2, 1592-1598.

206 Y. Zhang, Z. Shen, Y. Yu, L. Liu, G. Wang and A. Chen, J. Mater. Sci., 2018, 53, 12115-12122.

207 Y. Z. Zhang, Y. Wang, T. Cheng, W. Y. Lai, H. Pang and W. Huang, Chem. Soc. Rev., 2015, 44, 5181-5199.

208 X. Wen, X. Chen, N. Tian, J. Gong, J. Liu, M. H. Rümmeli, P. K. Chu, E. Mijiwska and T. Tang, Environ. Sci. Technol., 2014, 48, 4048-4055.

209 D. Puthusseri, V. Aravindan, B. Anothumakkool, S. Kurungot, S. Madhavi and S. Ogale, Small, 2014, 10, 4395-4402.

210 T. D. Dongale, P. R. Jadhav, G. J. Navathe, J. H. Kim, M. M. Karanjkar and P. S. Patil, Mater. Sci. Semicond. Process., 2015, 36, 43-48.

211 T. Weigert, Q. Tian and K. Lian, J. Power Sources, 2011, 196, 4061-4066.

212 S. Zhu, J. Li, L. Ma, C. He, E. Liu, F. He, C. Shi and N. Zhao, Mater. Lett., 2018, 233, 294-297.

213 S. I. Wong, J. Sunarso, B. T. Wong, H. Lin, A. Yu and B. Jia, J. Power Sources, 2018, 396, 182-206.

214 J. Xia, N. Zhang, S. Chong, D. Li, Y. Chen and C. Sun, Green Chem., 2018, 20, 694-700.

215 Z. Kang, C. Wu, L. Dong, W. Liu, J. Mou, J. Zhang, Z. Chang, B. Jiang, G. Wang, F. Kang and C. Xu, ACS Sustainable Chem. Eng., 2019, 7, 3364-3371.
216 C. Wang, D. Wu, H. Wang, Z. Gao, F. Xu and K. Jiang, J. Mater. Chem. A, 2018, 6, 1244-1254.

217 C. Jing, Y. Huang, L. Xia, Y. Chen, X. Wang, X. Liu, B. Dong, F. Dong, S. Li and Y. Zhang, Appl. Surf. Sci., 2019, 496, 143700.

218 A. Jain, C. Xu, S. Jayaraman, R. Balasubramanian, J. Y. Lee and M. P. Srinivasan, Microporous Mesoporous Mater., 2015, 218, 55-61.

219 C. Zhao, Y. Huang, C. Zhao, X. Shao and Z. Zhu, Electrochim. Acta, 2018, 291, 287-296.

220 S. Dong, X. He, H. Zhang, X. Xie, M. Yu, C. Yu, N. Xiao and J. Qiu, J. Mater. Chem. A, 2018, 6, 15954-15960.

221 M. Liu, J. Niu, Z. Zhang, M. Dou and F. Wang, Nano Energy, 2018, 51, 366-372.

222 S. Liu, Y. Zhao, B. Zhang, H. Xia, J. Zhou, W. Xie and H. Li, J. Power Sources, 2018, 381, 116-126.

223 B. K. Kim, S. Sy, A. Yu and J. Zhang, Handb. Clean Energy Syst., 2015, pp. 1-25.

224 M. D. Stoller and R. S. Ruoff, Energy Environ. Sci., 2010, 3, 1294-1301.

225 J. Zhang and X. S. Zhao, ChemSusChem, 2012, 5, 818-841.

226 C. G. Cameron, Springer Handbooks, 2017, vol. 38, pp. 563589.

227 G. A. Snook, P. Kao and A. S. Best, J. Power Sources, 2011, 196, 1-12.

228 W. Sun, R. Zheng and X. Chen, J. Power Sources, 2010, 195, 7120-7125.

229 L. Cui, J. Li and X. G. Zhang, J. Appl. Electrochem., 2009, 39, 1871-1876.

230 H. Liu, P. He, Z. Li, Y. Liu and J. Li, Electrochim. Acta, 2006, 51, 1925-1931.

231 H. K. Song, H. Y. Hwang, K. H. Lee and L. H. Dao, Electrochim. Acta, 2000, 45, 2241-2257.

232 B. K. Kim, V. Chabot and A. Yu, Electrochim. Acta, 2013, 109, 370-380.

233 N. Zhao, S. Wu, C. He, C. Shi, E. Liu, X. Du and J. Li, Mater. Lett., 2012, 87, 77-79.

234 G. Guidi, T. M. Undeland and Y. Hori, IEEJ Trans. Ind. Appl., 2008, 128, 418-423.

235 S. S. Williamson, P. A. Cassani, S. Lukic and B. Blunier, Energy storage, Elsevier Inc., 3rd edn, 2011.

236 L. Zhang and X. S. Zhao, Chem. Soc. Rev., 2009, 38, 2520-2531.

237 P. Simon, Nat. Mater., 2008, 7, 845-854.

238 Z. Tan, G. Chen and Y. Zhu, Nanocarbons Adv. Energy Storage, 2015, 1, 211-225.

239 M. Aghazadeh, M. Hosseinifard, B. Sabour and S. Dalvand, Appl. Surf. Sci., 2013, 287, 187-194.

240 R. Kumar, R. K. Singh, P. K. Dubey, D. P. Singh and R. M. Yadav, ACS Appl. Mater. Interfaces, 2015, 7, 15042-15251.

241 F. Zhou, Q. Liu, J. Gu, W. Zhang and D. Zhang, J. Power Sources, 2015, 273, 945-953.

242 S. Sahoo and A. K. Satpati, J. Electroanal. Chem., 2017, 801, 416-424.

243 L. Feng, Y. Zhu, H. Ding and C. Ni, J. Power Sources, 2014, 267, 430-444. 
244 A. M. Abioye, Z. A. Noorden and F. N. Ani, Electrochim. Acta, 2017, 225, 493-502.

245 C. Wang, J. Xu, M. F. Yuen, J. Zhang, Y. Li, X. Chen and W. Zhang, Adv. Funct. Mater., 2014, 24, 6372-6380.

246 X. Zhu, H. Dai, J. Hu, L. Ding and L. Jiang, J. Power Sources, 2012, 203, 243-249.

247 Y. Yan, B. Li, W. Guo, H. Pang and H. Xue, J. Power Sources, 2016, 329, 148-169.
248 Q. Yang, R. Bi, K. Chuen Yung and M. Pecht, Electrochim. Acta, 2017, 231, 125-134.

249 Q. Abbas, R. Raza, I. Shabbir and A. G. Olabi, J. Sci.: Adv. Mater. Devices, 2019, 4, 341-352.

250 H. P. Wu, D. W. He, Y. S. Wang, M. Fu, Z. L. Liu, J. G. Wang and H. T. Wang, Proc. - 2010 8th Int. Vac. Electron Sources Conf. Nanocarbon, IVESC 2010 NANOcarbon 2010, 2010, pp. 465-466. 